\title{
THE SOLAR FLARE PLASMA: OBSERVATION AND INTERPRETATION
}

\author{
G. A. DOSCHEK \\ E. O. Hulburt Center for Space Research, Naval Research Laboratory, \\ Washington, D.C. 20390, U.S.A.
}

\begin{abstract}
In the past several years, X-ray observations of the Sun made from rockets and satellites have demonstrated the existence of high temperature $\left(\sim 20 \times 10^{6}-\sim 100 \times 10^{6} \mathrm{~K}\right)$, low density plasmas associated with solar flare phenomena. In the hard X-ray range $(\lambda \lesssim 1 \AA)$, spectra of the flaring plasma have been obtained using proportional and scintillation counter detectors. It is possible from these data to determine the evolution of the hard X-ray flare spectrum as the burst progresses; and by assuming either a non-thermal or thermal (Maxwellian) electron distribution function, characteristic plasma parameters such as emission measure and temperature (for a thermal interpretation) can be determined. Thermal interpretations of hard X-ray data require temperatures of $\sim 100 \times 10^{6} \mathrm{~K}$.

In contrast, the soft $X$-ray flare spectrum $(1 \AA<\lambda<30 \AA)$ exhibits line emission from hydrogenlike and helium-like ions, e.g. $\mathrm{Ne}, \mathrm{Mg}, \mathrm{Al}, \mathrm{Si}, \ldots \mathrm{Fe}$, that indicates electron energies more characteristic of temperatures of $\sim 20 \times 10^{6} \mathrm{~K}$. Furthermore, line intensity ratios obtained during the course of an event show that the flare plasma can only be described satisfactorily by assuming a source composed of several different temperature regions; and that the emission measures and temperatures of these regions appear to change as the flare evolves. Temperatures are determined from line ratios of hydrogen-like to helium-like ions for a number of different elements, e.g., $\mathrm{S}, \mathrm{Si}$, and $\mathrm{Mg}$, and from the slope of the X-ray continuum which is assumed to be due to free-free and free-bound emission. There is no obvious indication in soft X-ray flare spectra of non-thermal processes, although accurate continuum measurements are difficult with the data obtained to date because of higher order diffraction effects due to the use of crystal spectrometers.

Soft X-ray flare spectra also show satellite lines of the hydrogen-like and helium-like ions, notably the $1 s^{2} 2 s^{2} S-1 s 2 s 2 p^{2} P$ transition of the lithium-like ion, and support the contention that in low density plasmas these lines are formed by dielectronic recombination to the helium-like ion. Also, series of allowed transitions of hydrogen-like and helium-like ions are strong, e.g., the Lyman series of $S$ up to Lyman- $\varepsilon$, and ratios of the higher member lines to the Lyman- $\alpha$ line can be compared with theoretical calculations of the relative line strengths obtained by assuming various processes of line formation.

This review will discuss the X-ray spectrum of solar flares from $\sim 250 \mathrm{keV}$ to $\sim 0.4 \mathrm{keV}$, but will be primarily concerned with the soft $\mathrm{X}$-ray spectrum and the interpretation of emission lines and continuum features that lie in this spectral range.
\end{abstract}

\section{Introduction}

Recently, considerable effort has been applied toward an understanding of the nature and origin of solar flares, e.g., Švestka (1970). This effort is partially due to the wealth of new data on the X-ray spectrum of flare plasmas obtained from instruments aboard artificial satellites. As in other fields of astronomical research, our knowledge of solar flares and flare related phenomena is proportional to the sophistication of observational techniques.

Early instruments flown by the Naval Research Laboratory on sounding rockets in the later 1940's and throughout the fifties, e.g., Kreplin (1961), demonstrated the marked correlation between optical $\mathrm{H} \alpha$ flares and enhanced X-ray emission. However, 
recording solar flare X-rays from rockets is difficult because of the necessity to launch during the phase of the flare over which substantial X-ray emission is expected; and conversely, a flare must occur sometime during the launch window designated for a particular experiment. Fortunately, the advent of orbiting spacecraft has eliminated much of this problem and provided thousands of hours of observation time over which solar flares have been studied in the ultraviolet and X-ray regions by a variety of experimental techniques. In particular, the series of NASA Orbiting Solar Observatories (OSO), the series of NRL Solar Radiation (SOLRAD) monitoring spacecraft, the U. S.S.R. Intercosmos and Cosmos satellites, Solar Satellites, and instrumentation flown by the Aerospace Corporation have contributed much to our understanding of solar activity and solar-terrestrial relationships.

This review will concentrate primarily on the soft X-ray spectrum of solar flares obtained from Bragg crystal spectrometers on orbiting spacecraft. Recent results concerning correlations of soft X-ray enhancements with other transient phenomena, e.g., radio bursts and $\mathrm{H} \propto$ flares, are only briefly discussed. The presentation and analysis of hard X-ray data $(\lambda \approx 1 \AA)$ are covered in another review given by De Feiter (1972).

\section{Instrumentation}

\section{A. BASIC TECHNIQUES}

Solar X-ray bursts and the X-ray spectra of flares have been investigated by several techniques. For wavelengths $\lesssim 1 \AA$ proportional counters and scintillation counters are employed; for wavelengths $\gtrsim 1 \AA$ and $\lesssim 40 \AA$, proportional counters, Bragg crystal spectrometers, ion chambers and $X$-ray photography by grazing incidence telescopes are useful. Grazing incidence grating spectrometers are also valuable at the longer wavelengths, but, because they have not been yet used in ffare studies, they will not be discussed. For soft X-ray wavelengths $(\lambda>1 \AA)$, each of the appropriate instruments has certain advantages and disadvantages. We discuss a few of the pertinent instrumental characteristics below.

Clearly, Bragg crystal spectrometers provide the highest spectral resolution at the shorter wavelengths ( $\lambda \lesssim 40 \AA$ ), revealing emission lines of highly ionized elements and a strong continuum, presumably due to free-free and free-bound processes. However, present crystal spectrometer experiments lack high time resolution since several minutes are required to complete a spectral scan, which is much too slow for impulsive flares. The poor time resolution primarily results from the dispersion of the crystal and the small values of the integrated reflectivity of the crystals that are most useful in the $1 \AA$ to $\sim 25 \AA$ range; typically, $R \lesssim 10^{-4}$. Consequently, there is a selective bias in the observations, i.e., intense $X$-ray flares are best observed, while smaller events, and the early heating phase of the large flares, are too weak in intensity to produce statistically significant counting rates.

A further disadvantage of present crystal spectrometers is the contamination of the continuum resulting from higher order diffraction. A fraction of the continuum at any wavelength $\lambda$ is due to emission at wavelengths $\lambda / n$, where $n=2,3, \ldots$. Higher order 
diffraction is serious for KAP crystals, which are used in the $6 \AA$ to $25 \AA$ region. Nevertheless, this problem is not insurmountable, e.g., Duwez (1965) has investigated higher order diffraction for several crystals, and Unzicker (1971) is studying higher order diffraction from LiF, EDDT, and KAP crystals. Since these three crystals were used in the NRL OSO-6 experiment, the results of the laboratory investigation should enable the NRL group to derive reasonably accurate line to continuum ratios and relative line intensities.

Conversely, proportional counters have good time resolution $(<1 \mathrm{~s})$, but poor spectral resolution compared to crystal spectrometers. However, Culhane and Phillips (1970a); and Phillips and Culhane (1971) have numerically resolved the ironline emission feature $\sim 1.9 \AA$ from the continuum in their proportional counter data, and, from observations of this feature and the continuum, electron temperatures and emission measures have been derived under the assumption of thermal equilibrium. Nevertheless, because of coarse spectral resolution, proportional counters are not suitable for a detailed study of atomic processes in flare plasmas. Because of their high time resolution, they are useful for correlating soft X-ray emission with other solar activity, and for providing an overall view of the evolution of individual events, e.g., determining average temperatures and emission measures as the flare progresses.

Ion chambers are of restricted usefulness in studying the detailed behavior of flare plasmas because they are broad band detectors and provide only limited spectral information. In order to calculate the absolute flux in any of the spectral bands to which ion chambers are typically sensitive, e.g., $1 \AA$ to $8 \AA$, a theoretical source spectrum must be assumed and convoluted with the chamber efficiencies. Different theoretical spectra often lead to different values for the derived fluxes in the energy bands.

However, ion chambers are very useful for providing continuous monitoring of solar X-ray activity and for correlating this activity with other transient phenomena. There now exist continuous monitoring data extending from July 1968 to the present. These plots are of use to researchers engaged in studies of X-ray flares because the large events may be quickly identified in time, so that a systematic and tedious search through experimenter tapes for large events is not necessary. Sometimes, if only partial coverage is obtainable from crystal spectrometer observations of a particular event, the broadband data provide useful information on the general development of the flare. A further result derived from ion chamber data which is relevant to flare plasmas is described in the next section.

One final instrumental technique should be mentioned. Although considerable information is provided by broadband and crystal spectrometer instruments, a thorough understanding of soft X-ray bursts will require a knowledge of the geometry of the emitting plasma and information on the magnetic confinement. This information can be provided in part by photographs taken with grazing incidence X-ray telescopes. From a rocket flight (Vaiana et al., 1968), the American Science and Engineering (AS \& E) group has obtained high resolution $\left(<5^{\prime \prime} \mathrm{sec}\right)$ photographs of a flare, and their bearing on the spectral data will be discussed in Section 6 . 


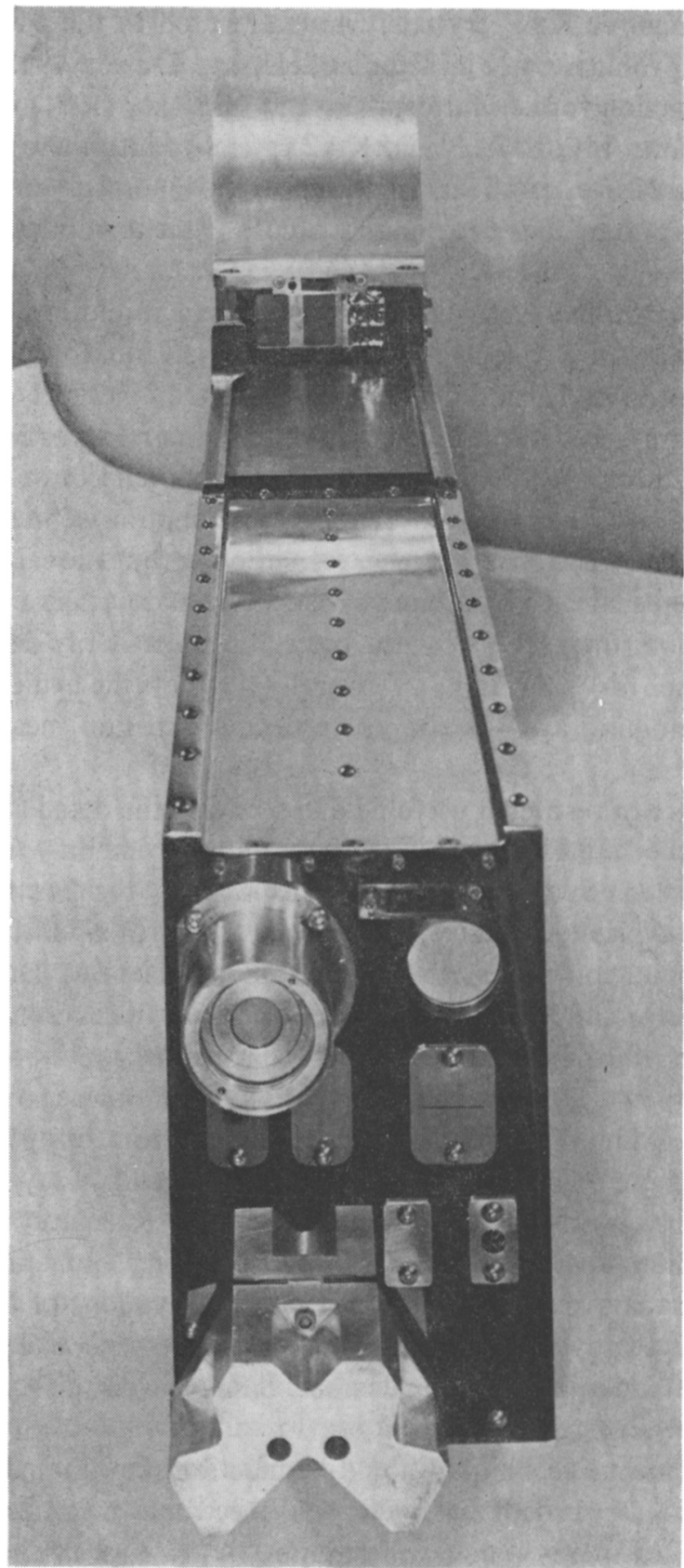

Fig. 1. The assembled NRL experiment package flown on OSO 6. Instruments include three Bragg crystal spectrometers, a proportional counter and a scintillation counter, a Geiger counter that monitors solar activity in the $8 \AA-15 \AA$ band, and Geiger counters collimated by pinholes and slits in order to construct X-ray maps of the Sun during the raster mode of the spacecraft. 
B. THE NAVAL RESEARCH LABORATORY CRYSTAL SPECTROMETER EXPERIMENTS

All the crystal spectrometers thus far flown on orbiting spacecraft are similar in concept and design. The NRL instruments aboard OSO 6 are briefly described below. These instruments are similar to instruments flown by other investigators.

The NRL package in the pointed section of OSO 6 consists in part of three Bragg crystal spectrometers that scan the X-ray spectrum from 0.6 to $\sim 14 \AA$. A LiF crystal is used in the $0.6 \AA$ to $4 \AA$ range $(2 d=4.016 \AA)$; an EDDT crystal covers the $1 \AA$ to $8.5 \AA$ region $(2 d=8.800 \AA)$; and a KAP crystal scans the $6 \AA$ to $\sim 14 \AA$ window $(2 d=26.827 \AA)$. The three instruments are uncollimated and therefore record emission from the entire Sun. Nevertheless, intense X-ray flares produce orders of magnitude more flux than neighboring active regions. Therefore, blending of non-related X-ray sources is seldom a problem in studying large events.

Mylar and parylene filters coated with aluminum are used to shield the crystals and argon-filled, bromine quenched Geiger counters from low energy particle contamination and to reduce solar heating of the instruments. Particle interference from the Van Allen belts is easily recognizable, and because of the bromine quench, a mica window was used on the Geiger counters.

The crystals and detectors are mounted on a common assembly and are rotated through the Bragg angles by a stepping motor. For OSO 6 the three spectral ranges may be scanned in either seven minutes (slow scan), or in two minutes (fast scan). Therefore it is clear that, if appreciable changes in X-ray emission occur on time scales of a few minutes, the observed spectrum will reflect both spectral characteristics of the source and temporal changes. This is almost always the case for flares observed by the NRL group.

In the NRL instrument data are accumulated and stored over an effective time interval of $0.64 \mathrm{~s}$. The stepping motor drives the crystals in angular increments of six arc minutes, data readout occurring at the end of each step. Therefore, in the slow scan, high resolution mode, each spectrum consists of 660 steps, or 660 data points. Each datum represents the true spectral characteristics of the source, at the time of measurement, modified by the transmission of the filter, the response characteristics of the crystal (rocking curve, integrated reflectivity), the quantum efficiency of the detector, and the effects of higher order diffraction.

If the rocking curves of the crystals were smaller than the angular stepping increments of the motor $\left(6^{\prime}\right)$, it would be possible to skip over spectral lines. To insure that this could not happen, the LiF and EDDT crystals were bent on a right circular cylinder; a procedure which deforms and widens the approximately Gaussian rocking curve of a flat crystal into a shape similar to a square wave. The full widths of the bent rocking curves are six arc minutes and therefore all spectral lines are detectable. A photograph of the assembled NRL instrument is shown in Figure 1 and a typical rocking curve of a bent crystal and its effect on spectral lines is illustrated in Figure 2. Figures 3 and 4 show approximate instrumental efficiencies for the NRL LiF and EDDT spectrometers flown on OSO 4. 


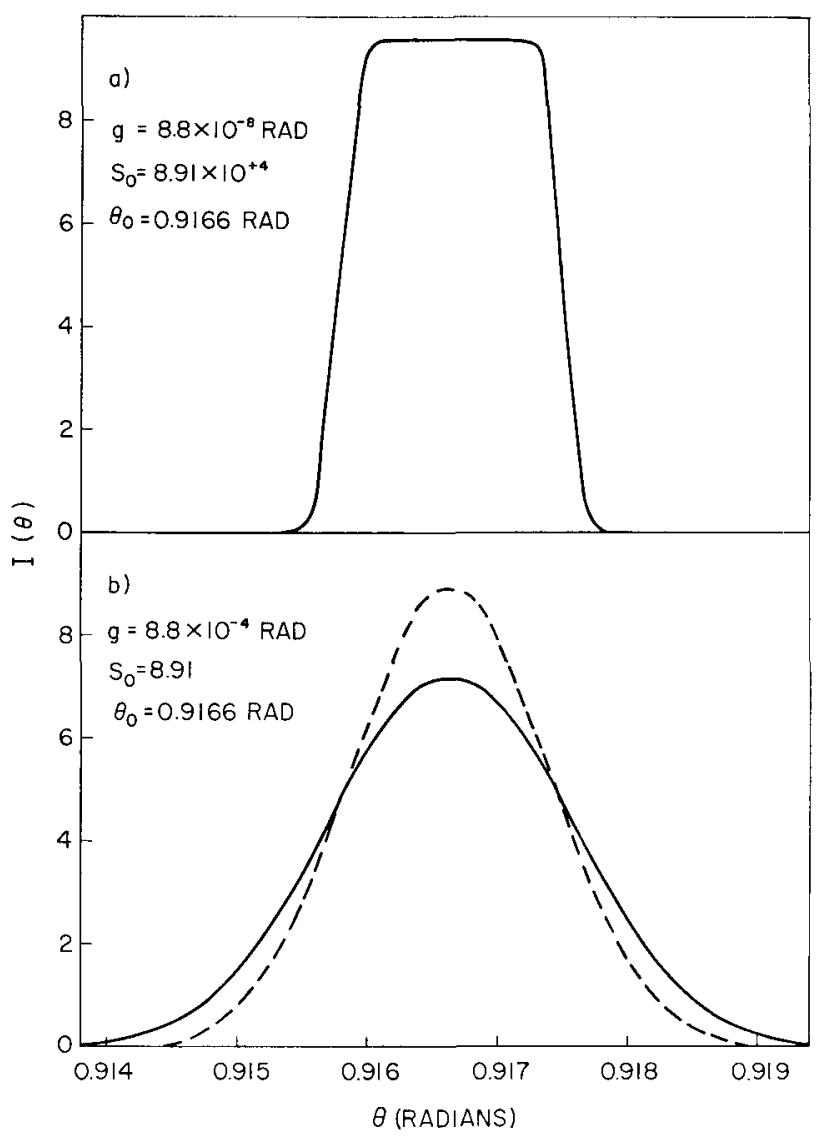

Fig. 2. Relative effect of crystal bending on line shape of the source. In Figure 2a, the source line shape is approximately a $\delta$-function and in $2 \mathrm{~b}$ a Doppler line shape is assumed (dashed curve). In both $2 \mathrm{a}$ and $2 \mathrm{~b}$, the energies of the lines are equal, but their widths and amplitudes differ. Solid curves represent the line shape after crystal reflection. The quantities $g, S_{0}$, and $\theta_{0}$, represent respectively the half width at half maximum of the line, the amplitude of the line at line center, and the angle corresponding to the wavelength of line center.

Two other instrumental effects are important. First, because the pointed section of the OSO 6 spacecraft can scan the solar disk in a raster pattern (large and small raster modes), the spectrometer data are unreliable when the pointed section returns to start of raster, in that the angle between source and crystal is uncertain. However, it is always possible to detect these raster maneuvers in the data. Nevertheless, the best spectra are obtained when the instrument is in pointed mode, i.e., the pointed section is pointed at the center of the solar disk to within the tolerance of the spacecraft $\left(\sim 20^{\prime \prime}\right)$ throughout the observation period.

Secondly, the first order wavelength corresponding to each Bragg angle is given by Bragg's law:

$$
\lambda=2 d \sin \theta
$$


where $d$ is the interatomic spacing of the crystal planes. Consequently, the wavelength resolution is given by

$$
\delta \lambda=\left[(2 d)^{2}-\lambda^{2}\right]^{1 / 2} \delta \theta,
$$

where $\delta 0=6^{\prime}$ (due to bending) for the NRL instruments and is constant over the spectral range scanned. From Equation (2) it follows that spectral resolution is highest at

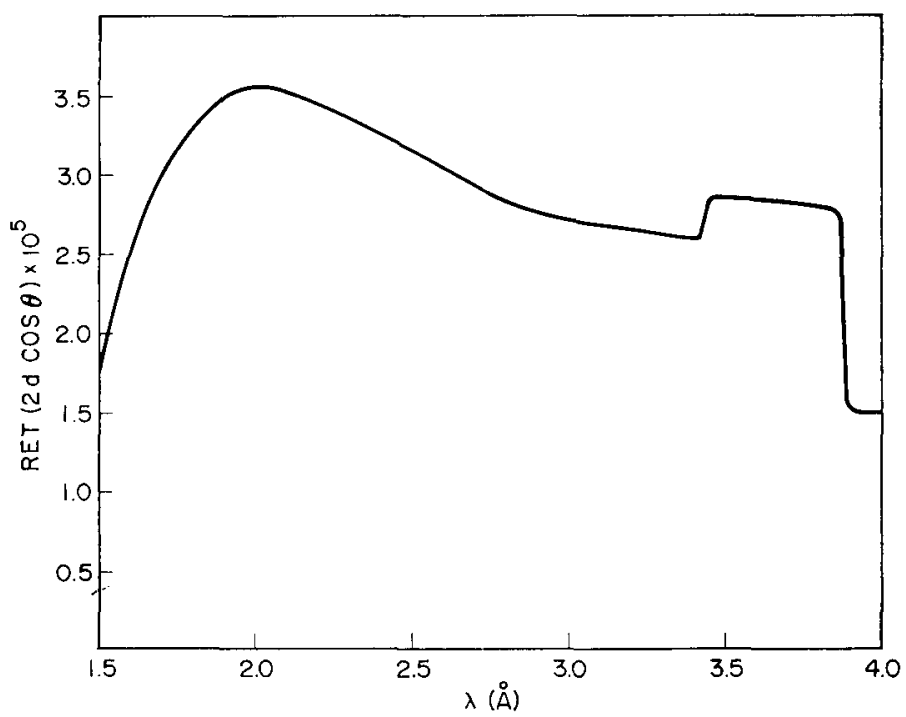

Fig. 3. Product of the integrated reflectivity, quantum efficiency, and transmission of the filter (RET) multiplied by $2 d \cos \theta$ for the NRL LiF spectrometer aboard OSO 4 .

The curve is only approximate.

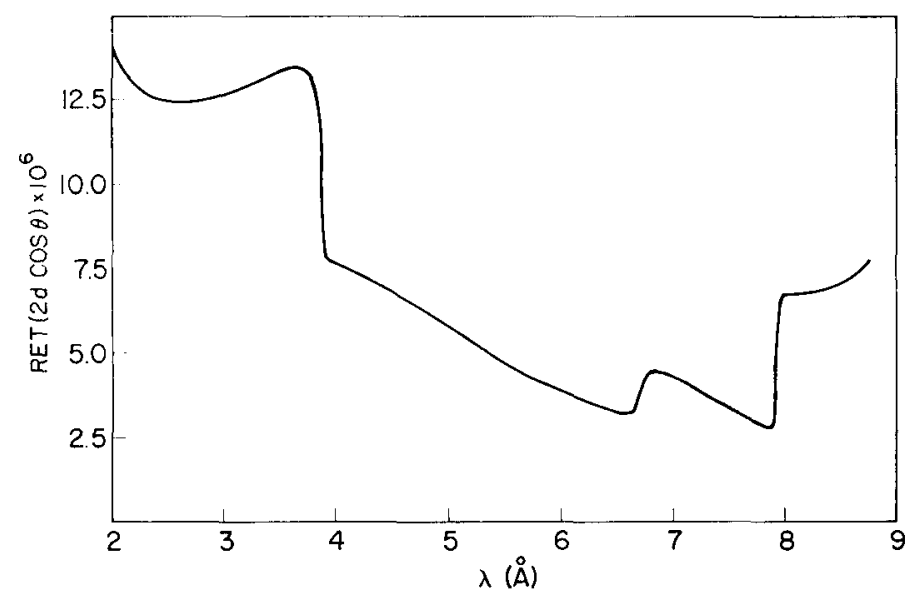

Fig. 4. Product of the integrated reflectivity, quantum efficiency, and transmission of the filter (RET) multiplied by $2 d \cos \theta$ for the NRL EDDT spectrometer aboard OSO 4 . The curve is only approximate. 
long wavelengths, i.e., near 3.7 for LiF, near $8.2 \AA$ for EDDT, and near $\sim 14 \AA$ for KAP. Also, it follows from the $2 d$ spacings and spectral ranges of the crystals that resolution is highest for the LiF crystal and lowest for the KAP crystal. In one sense this is unfortunate, for the flare spectrum increases in complexity with increasing wavelength.

\section{Solar Flare Spectra - Description and Line Identification}

\section{A. BROADBAND RESULTS}

Many significant observations and results have been obtained with broadband instrumentation. These are well-described in the literature, e.g., Culhane et al. (1969), Teske and Thomas (1969), Culhane and Phillips (1970a, b), Horan (1971), Thomas and Teske (1971), Drake (1971), and Hudson and Ohki (1971). Several of the more important findings reported in these papers are:

(1) Soft X-ray bursts exhibit large variations in the total time intervals over which the bursts occur. Some bursts are impulsive while others are comparatively long-lived. Impulsive events have nearly identical rise and decay profiles, usually on a time-scale of a few minutes. Long-lived events exhibit a fairly rapid rise in flux ( $\sim$ a few minutes), but a substantially longer decay $(\sim 1 \mathrm{~h})$. An extreme example of the latter occurred on 10 April 1969. In the SOLRAD data of Figure 5 the decay phase of this event can be traced over at least $24 \mathrm{~h}$.

In between these extremes, there is a continuous gradation of time-scales, and there does not appear to be any physical differences between impulsive and long-lived events (Drake, 1971).

(2) Soft X-ray bursts usually correlate well with $\mathrm{H} \alpha$ events with respect to start times, peak flux, and decay times. (However, statistical problems abound because the description of $\mathrm{H} \alpha$ flares usually depends heavily on value judgments of observers, and interpretations of the X-ray observations.)

(3) It may be that some soft X-ray events are preceded by a precursor, or enhancement of soft X-rays indicative of an $\sim 10 \times 10^{6} \mathrm{~K}$ plasma (Culhane and Phillips, 1970b).

(4) An enhancement of microwave emission usually accompanies intense soft X-ray bursts (Hudson and Ohki, 1971).

(5) The peak flux in different energy bands, e.g., $0.5 \AA$ to $3 \AA, 1 \AA$ to $8 \AA$, occurs at different times; the peak in lower energy channels always occurring later than the peak flux in higher energy channels.

(6) Recently, Horan (1971) has derived results relevant to flare spectra from an analysis of ion chamber data of seventeen flares obtained by an NRL instrument aboard OGO 4. Horan derived qualitative relationships concerning the electron temperature of the plasma, the X-ray flux in particular energy bands, e.g., $1 \AA$ to $8 \AA$, and the emission measure of the plasma. Typical results of his analysis are shown in Figure 6 for the flare of October 29, 1967. The temperatures and emission measures were obtained as follows: Horan assumed that the flare plasma is isothermal, and that emission in the $0.5 \AA$ to $3 \AA$ band and the $1 \AA$ to $8 \AA$ band is primarily due to free-free and freebound processes. A small correction was applied for line emission. Then, using theo- 


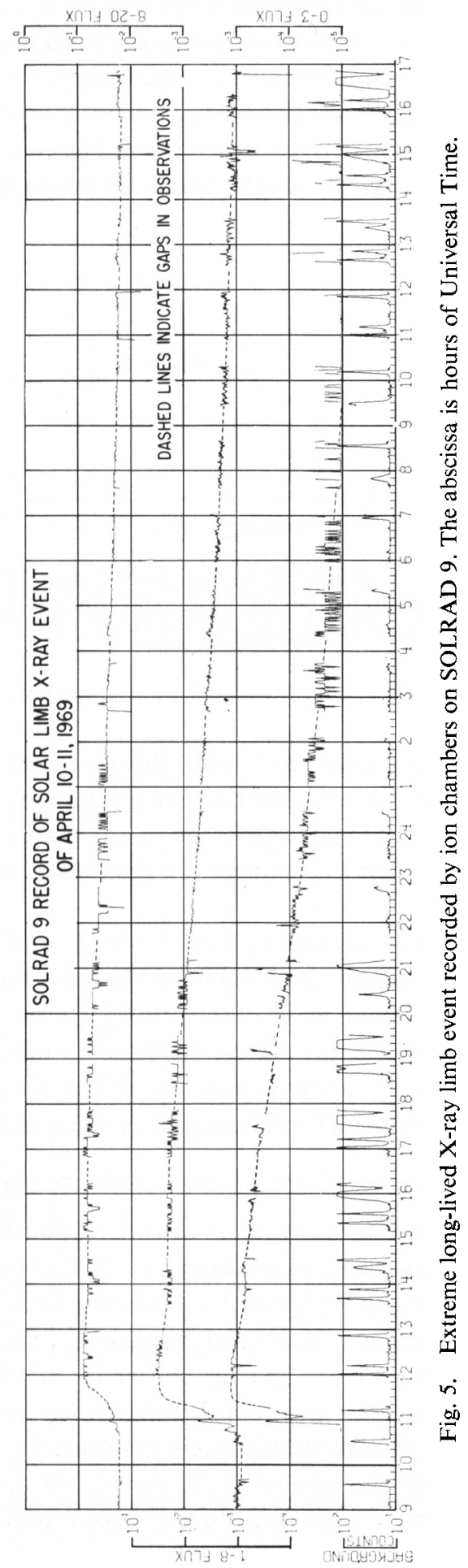


retical cross-sections for free-free and free-bound processes, and calculations of ionization equilibrium and relative element abundances (Pottasch, 1964), Horan derived the average flare temperature as a function of time from the ratio of the $0.5 \AA$ to $3 \AA$ flux to the $1 \AA$ to $8 \AA$ flux. Since the flare plasma is probably multithermal (Meekins et al., 1970), the temperatures so derived are averages. The emission measure may then be calculated from the flux in either energy band and the computed temperature.

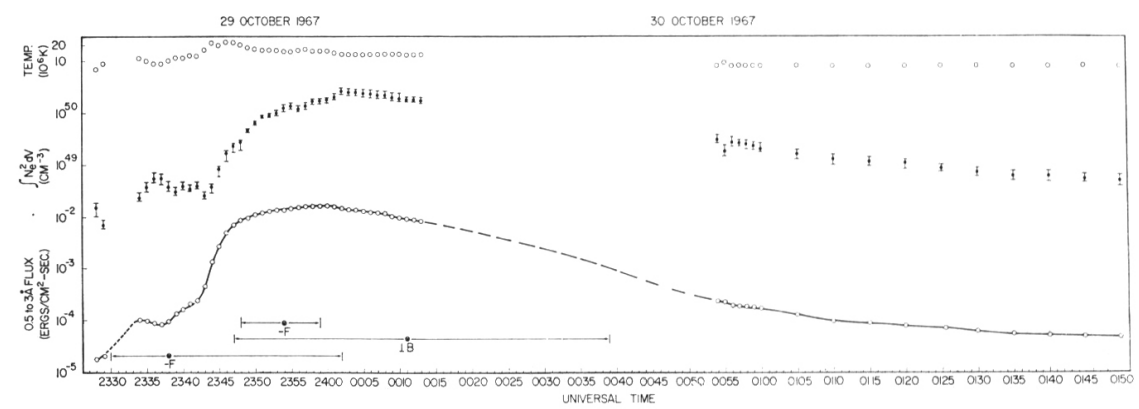

Fig. 6. Ion chamber data and results obtained by Horan (1971) from NRL instruments on OGO 4. The temperatures and emission measures were obtained as described in the text. The times of optical events are also indicated in the figure.

These results are necessarily crude, but show three important features of soft X-ray emission during flares:

(1) The temperature rises rapidly and peaks before either the X-ray flux or the emission measure, implying that a greater number of electrons is involved in the emission as the flare evolves, even though the average energy per unit volume is decreasing.

(2) The X-ray flux peaks after the temperature peaks and before the emission measure peaks.

(3) The emission measure rises rapidly by a few orders of magnitude during the initial phase of the event, reaches a peak value and remains fairly constant for a considerable time ( $\gtrsim 30 \mathrm{~min}$ ). From these observations, Horan (1971) concluded that during the initial phases of soft X-ray events, the behavior of the X-ray flux is primarily determined by the emission measure, and that during the decay phases of flares, the behavior of the flux is principally determined by the decreasing temperature.

\section{B. CRYSTAL SPECTROMETER OBSERVATIONS - LINE IDENTIFICATIONS}

Crystal spectrometers designed to study solar X-ray flares and active regions have been flown on spacecraft by Neupert and his colleagues at the Goddard Space Flight Center (OSO 3, OSO 5), by Walker and Rugge at the Aerospace Corporation (OV 1 10, OV 1 17), by Friedman and his group at the Naval Research Laboratory (OSO 4, OSO 6), and more recently by Mandelshtam and his colleagues at the Lebedev Institute in Moscow (Intercosmos 4). Most of the published flare data has been obtained by the Goddard and NRL groups; Walker and Rugge have primarily observed active regions and cooler flares and the Russian results are so recent that little has been published.

Neupert et al. (1967) reported the first observation of an intense soft X-ray flare. 
Previously, crystal spectrometers flown on rockets and satellites (Evans et al., 1967; Fritz et al., 1967; Rugge and Walker, 1968) had only recorded emission from active regions. A typical Goddard flare spectrum in the $1 \AA$ to $20 \AA$ range is shown in Figures 7 and 8 , with line identifications assigned by the Goddard group. The lower scan

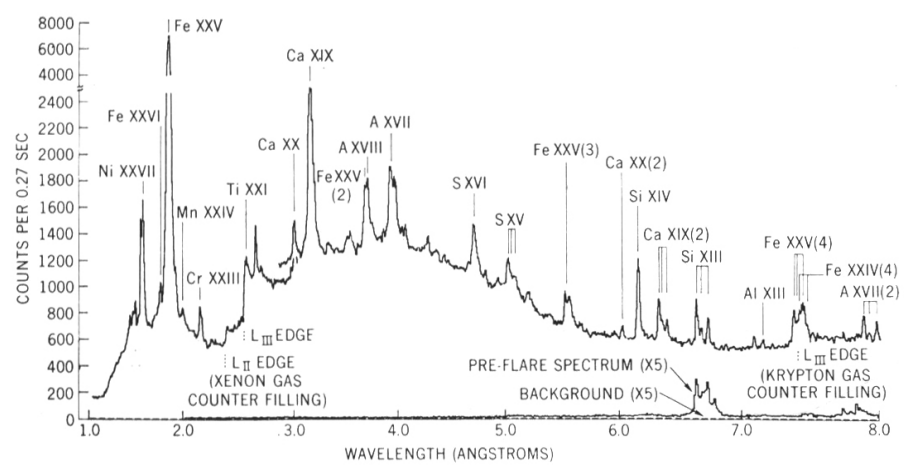

Fig. 7. The soft X-ray spectrum of an intense solar flare obtained by crystal spectrometers on OSO 5 (February 27, 1969). The spectrum is a composite of spectra obtained with LiF and ADP spectrometers, and is not corrected for instrumental efficiencies. Spectrum courtesy of W. M. Neupert, Goddard Space Flight Center.

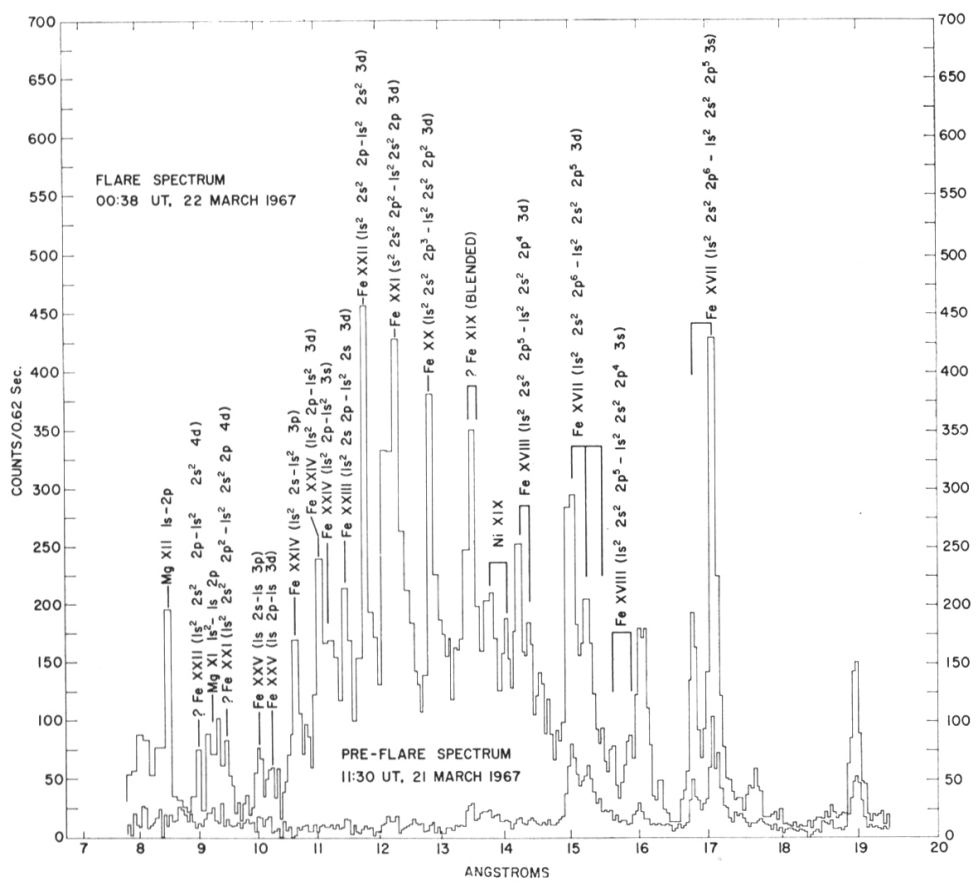

Fig. 8. Soft X-ray flare spectrum obtained by a KAP crystal spectrometer on OSO 5. The complexity of the spectrum above $8 \AA$ is apparent. Note the large number of lines identified as FexXFexxv. In the pre-flare spectrum, only the Fexvil lines are present. The emission $\sim 19 \AA$ is the Lyman- $\alpha$ line of O VIII. The spectrum is not corrected for instrumental efficiencies. Spectrum courtesy of W. M. Neupert, Goddard Space Flight Center. 

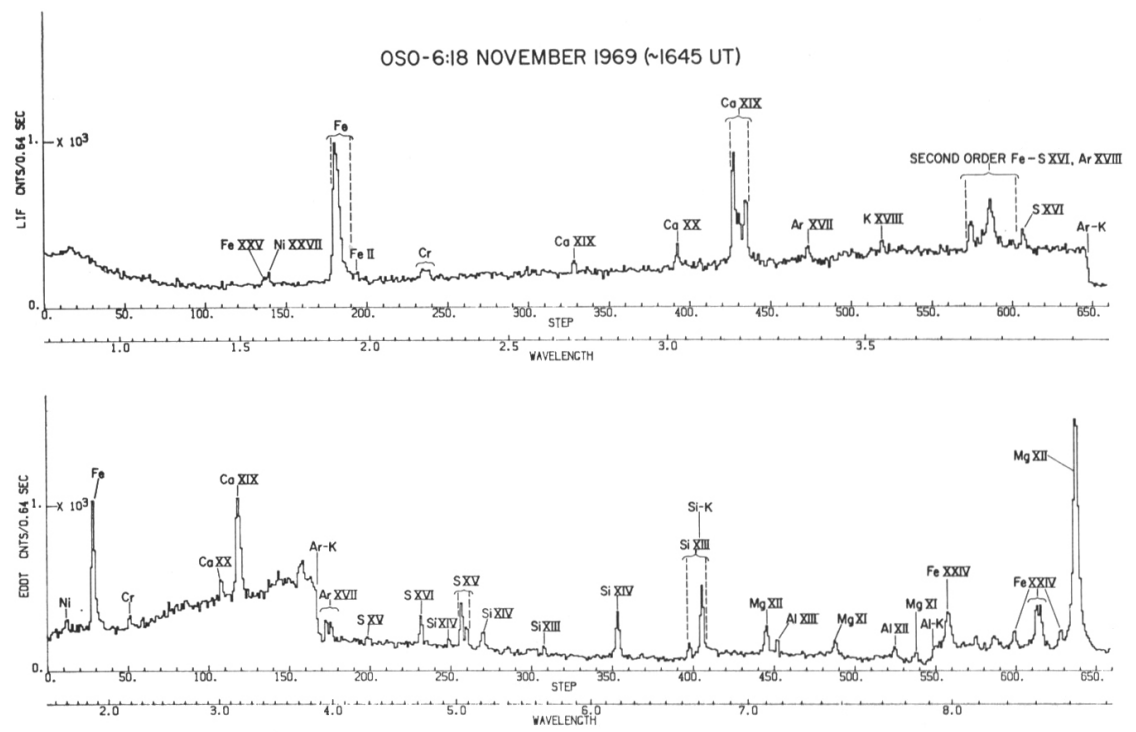

Fig. 9. Spectra of a large soft X-ray flare obtained by NRL crystal spectrometers on OSO 6. A comparison of these scans with Figure 7 reveals many emission features in common. This flare may be cooler than the flare in Figure 7 since no emission of Fexxvi appears to be present. The spectra are not corrected for instrumental efficiencies.

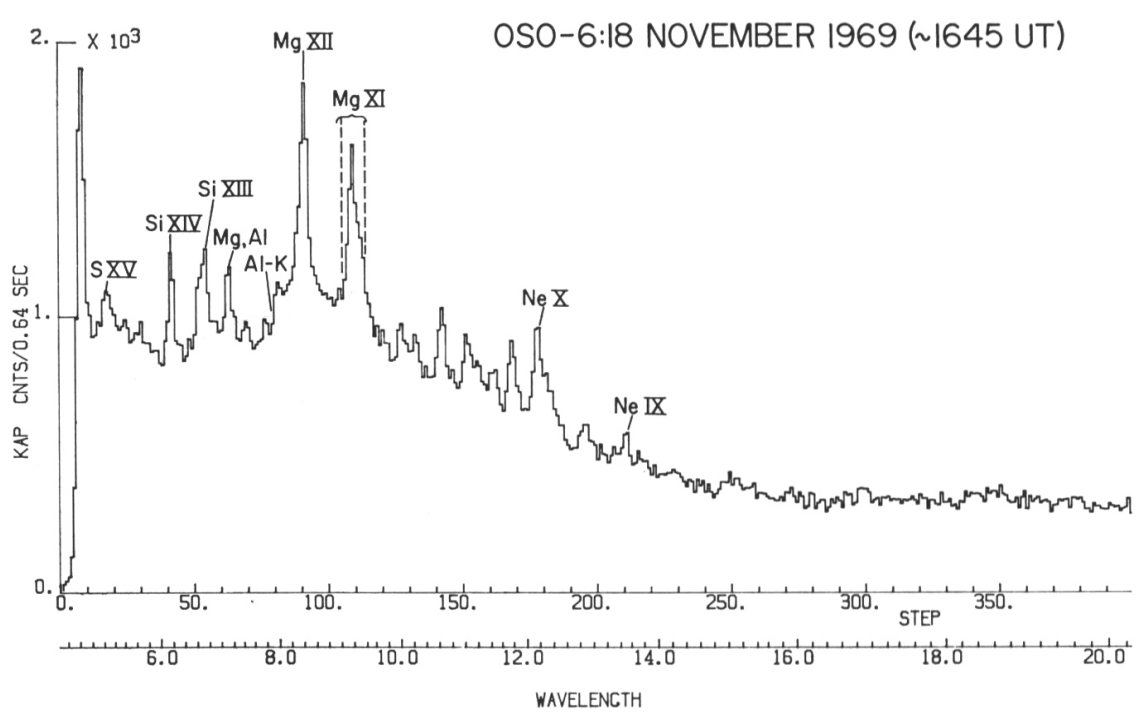

Fig. 10. Soft X-ray flare spectrum obtained by an NRL KAP erystal spectrometer on OSO 6 . Emission due to MgXI $(\sim 9.17 \AA)$ and $\mathrm{MgXII}(8.42 \AA)$ is quite prominent. The MgXI emission is a blend of the resonance, intercombination, and forbidden lines. Note the poor resolution of the KAP spectrum compared to the EDDT spectrum in Figure 9. The spectrum is not corrected for instrumental efficiencies. 
in Figure 8 is a pre-flare spectrum, and during the intense flare shown in the upper scan, many lines produced by ions in high ionization stages appear that are absent in the pre-flare spectrum. Also strong continuum emission is evident in the flare spectrum. The crystal used to obtain the spectra was KAP; at shorter wavelengths the Goddard group employed a LiF crystal $(1 \AA \leq \lambda \leq 3 \AA$ ) and an ADP crystal ( $3 \AA \leq \lambda \leq 8 \AA$ ). Figure 7 is a composite of the LiF and ADP spectra, and shows prominent emission lines of hydrogenic aluminum, silicon, sulfur, and calcium, and strong lines produced by helium-like ions of aluminum, silicon, sulfur, argon, calcium and iron.

NRL spectra of a large flare are shown in Figures 9 and 10. Neither the Goddard nor the NRL spectra are corrected for instrumental effects. In the NRL spectra, instrumental edges due to potassium, argon, silicon, and aluminum appear at $3.436 \AA$, $3.871 \AA, 6.723 \AA$, and $7.951 \AA$, respectively. A comparison of Figures 7, 8, 9, and 10 reveals many spectral features in common for these different flares. However, the presence of Fexxvı in the Goddard spectrum $(\simeq 1.79 \AA)$ indicates that the Goddard flare may be hotter than the NRL event.

A cooler flare is shown in Figures 11 and 12. The data were obtained by Walker and Rugge from their experiments on the OV1 10 and OV1 17 spacecraft. It is instructive to compare the NRL EDDT and KAP spectra (Figures 9 and 10) with the Walker and Rugge data in Figures 11 and 12. The enhancement of continuum and line emission at shorter wavelengths in the NRL spectrum shows that the NRL event was considerably hotter than the Walker and Rugge flare. Note also that Walker and Rugge do not use a mica window on their detector. Consequently, the silicon instrumental edge at $6.723 \AA$ is absent in their spectrum. In the NRL spectrum, the forbidden line of

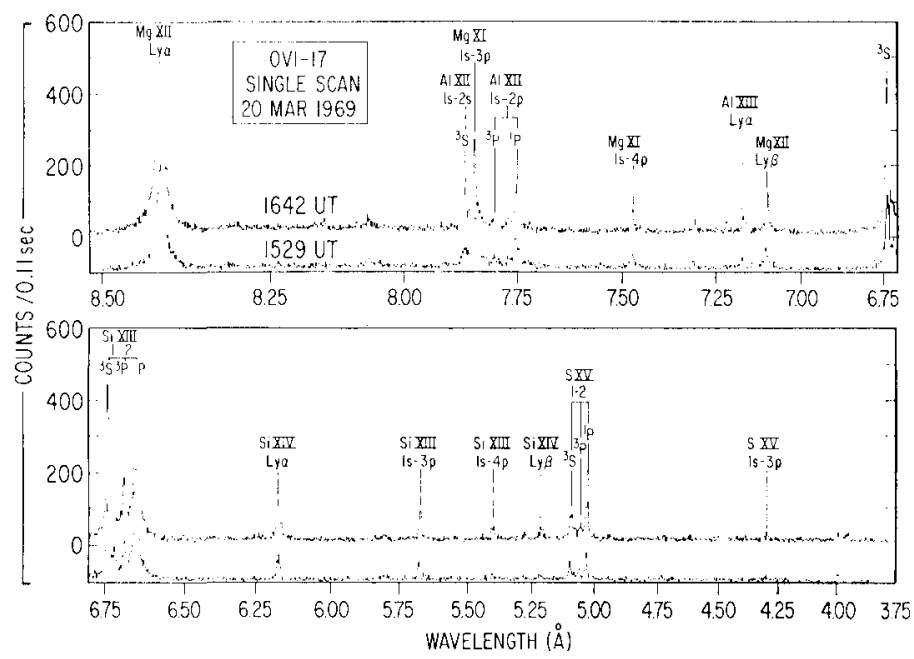

Fig. 11. Soft $\mathrm{X}$-ray EDDT spectrum of a relatively cool flare $\left(T \sim 10 \times 10^{6} \mathrm{~K}\right)$ uncorrected for instrumental efficiencies. From a comparison of the resonance lines of SixIII and SixIV in Figures 9 and 11 , it is clear that the flare spectrum in Figure 9 is indicative of a much hotter plasma $\left(T \sim 25 \times 10^{6}\right)$ than the spectrum of Figure 11 indicates. This is supported by the absence of SXVI and FexxIV lines in this spectrum. Spectrum courtesy of A. B. C. Walker, Jr., Aerospace Corporation. 


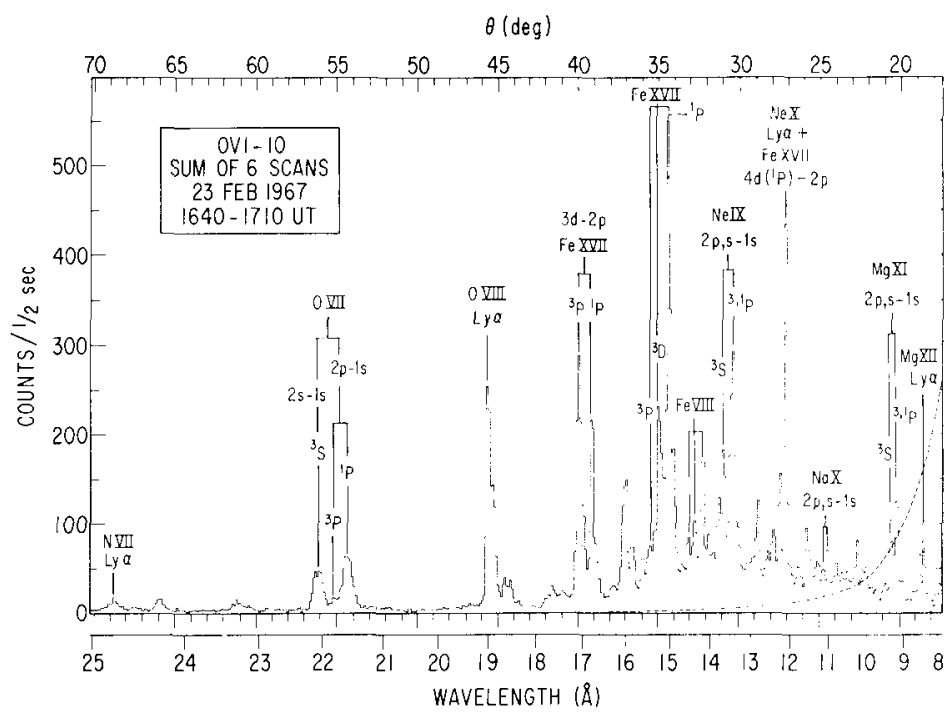

Fig. 12. Cool X-ray flare KAP spectrum uncorrected for instrumental efficiencies. Note the weakness or absence of FexxIII and Fexxiv lines in this spectrum and the strength of the OVIII and Fexvil lines.

Compare with Figures 8 and 10. courtesy of A. B. C. Walker, Jr., Aerospace Corporation.

Si XIII at $6.74 \AA$ appears stronger than the resonance lines of SixIII because the forbidden line lies on the long-wavelength side of the silicon edge.

From observations of a large number of flares similar to those illustrated (Figures 7-12), wavelength lists with identifications have been published by Meekins et al. (1970), Neupert (197la), and Walker and Rugge (1969, 1970, 1971). Table I gives a composite listing of all the lines below $\sim 8.5 \AA$ reported in flare spectra published to date. The wavelengths and identifications were obtained from the references cited above. In addition, the NRL wavelength list (OSO 4) has been considerably revised and expanded due to new data obtained from OSO 6. Only wavelengths through $\sim 8.5 \AA$ have been listed because the complexity of the flare spectrum above $9 \AA$, combined with the relatively coarse resolving power of the KAP crystal, makes even tentative identifications difficult for many of the lines found in this spectral region during intense flares. Wavelength lists for $\lambda \gtrsim 9 \AA$ can be found in Walker and Rugge (1969), and identifications of lines seen during very hot events are shown in the Goddard spectrum in Figure 8. Several of the more prominent lines found above $9 \AA$ are due to MgXI; iron stages, Fexviı to Fexxiv; NeIX, Nex; O viI, O viII; and Nvil (see Figures 8,10 , and 12).

Table I shows about 120 lines between 0.7 and $\sim 8.5 \AA$. Some of the identifications are only tentative, and these are indicated by asterisks. Identifications are based primarily on the following observations:

(1) Theoretical and extrapolated wavelength coincidences with observed lines.

(2) Expectation of the transition based on element abundance, electron temperature, and flux variations during the evolution of the flare. 
TABLE I

Solar flare emission lines

\begin{tabular}{|c|c|c|c|c|c|}
\hline \multirow[t]{2}{*}{ Wavelength } & \multirow{2}{*}{$(\AA)$} & \multirow{2}{*}{$\begin{array}{l}{[1,2,3]} \\
8.550\end{array}$} & \multirow{2}{*}{$\begin{array}{l}\text { Predicted } \\
\text { Wavelength }(\AA) \\
8.548\end{array}$} & \multicolumn{2}{|l|}{ Identification } \\
\hline & & & & $* \operatorname{MgxI}\left(1 s 2 p^{1} P-2 p^{21} D\right)$ & \\
\hline - & - & 8.518 & $8.519[3]$ & $* \mathrm{Mg} \times \mathrm{I}\left(1 s 2 s^{3} S-2 s 2 p^{3} P\right)$ & \\
\hline 8.490 & - & - & $8.488[3]$ & ${ }^{*} \operatorname{Mg} \times I\left(1 s 2 s^{1} S-2 s 2 p^{1} P\right)$ & [15] \\
\hline 8.458 & - & - & 8.449 [3] & ${ }^{*} \operatorname{Mg} \times \mathbf{I}\left(1 s 2 p^{1} P-2 p^{21} S\right)$ & [14] \\
\hline 8.421 & 8.421 & 8.421 & $8.421[4]$ & $\operatorname{MgxII}\left(1 s^{2} S_{1 / 2}-2 p^{2} P_{1 / 2,3 / 2}\right)$ & [13] \\
\hline 8.396 & - & - & $?$ & $?$ & \\
\hline 8.378 & - & - & $8.368[10]$ & $\operatorname{FexxIV}\left(1 s^{2} 2 p^{2} P_{3,2}-1 s^{2} 4 s^{2} S_{1 / 2}\right)$ & [12] \\
\hline 8.317 & - & - & $8.311[10]$ & $\operatorname{FexxIv}\left(1 s^{2} 2 p^{2} P_{3 / 2}-1 s^{2} 4 d^{2} D_{5 / 2}\right)$ & [11] \\
\hline 8.307 & - & - & $8.306[10]$ & ${ }^{*} \mathrm{FeXXIII}\left(1 s^{2} 2 s^{2}{ }^{1} S-1 s^{2} 2 s 4 p^{1} P\right)$ & [10] \\
\hline 8.290 & - & - & $8.279[10]$ & $\operatorname{FexXIv}\left(1 s^{2} 2 p^{2} P_{1 / 2}-1 s^{2} 4 s^{2} S_{1 / 2}\right)$ & [9] \\
\hline 8.254 & - & - & $?$ & $?$ & [8] \\
\hline 8.233 & - & - & $8.225[10]$ & $\operatorname{FexxIv}\left(1 s^{2} 2 p^{2} P_{1 / 2}-1 s^{2} 4 d^{2} D_{3 / 2}\right)$ & [7] \\
\hline 8.208 & -. & - & $?$ & $?$ & [6] \\
\hline 8.170 & - & - & $?$ & $?$ & [5] \\
\hline 8.157 & - & - & $?$ & $?$ & [5] \\
\hline 8.094 & - & 8.068 & $8.073[3]$ & $\begin{array}{l}{ }^{*} \operatorname{Mgx}\left(1 s^{2} 2 p^{2} P-1 s 2 p 3 p^{2} S\right) \\
{ }^{*} \operatorname{Mgx}\left(1 s^{2} 2 p^{2} P-1 s 2 p 3 p^{2} D\right)\end{array}$ & $\begin{array}{l}{[4]} \\
{[4]}\end{array}$ \\
\hline 8.073 & - & 8.034 & $8.039[3]$ & ${ }^{*} \operatorname{Mgx}\left(1 s^{2} 2 s^{2} S-1 s 2 s 3 p^{2} P\right)$ & [3] \\
\hline 7.990 & - & - & $7.982[10]$ & $\operatorname{FexxIv}\left(1 s^{2} 2 s^{2} S-1 s^{2} 4 p^{2} P\right)$ & [2] \\
\hline 7.935 & - & - & $?$ & $?$ & [1] \\
\hline- & 7.897 & 7.873 & $7.871[7]$ & $\operatorname{Alxu}\left(1 s^{21} S-1 s 2 s^{3} S\right)$ & \\
\hline $7.864(?)$ & - & 7.864 & $7.863[3]$ & ${ }^{*} \operatorname{MgXi}\left(1 s^{2}{ }^{1} S-1 s 3 p{ }^{3} P\right)$ & \\
\hline 7.854 & 7.857 & 7.850 & $7.850[6]$ & $\operatorname{Mg} \times I\left(1 s^{2}{ }^{1} S-1 s 3 p^{1} P\right)$ & \\
\hline- & - & 7.827 & $?$ & $?$ & \\
\hline- & 7.795 & 7.805 & $7.807[3]$ & $\operatorname{AlxII}\left(1 s^{21} S-1 s 2 p^{3} P\right)$ & \\
\hline- & - & 7.776 & $7.787[3]$ & $\begin{array}{l}{ }^{*} \mathrm{AIxI}\left(1 s^{2} 3 p^{2} P-1 \mathrm{~s} 2 p 3 p^{2} D\right) \\
{ }^{*} \mathrm{AlXI}\left(1 s^{2} 3 p^{2} P-1 s 2 p 3 p^{2} S\right)\end{array}$ & \\
\hline 7.755 & 7.761 & 7.759 & $7.756[6]$ & $\mathrm{Al} \times 11\left(1 s^{21} S-1 \mathrm{~s} 2 p^{1} P\right)$ & \\
\hline 7.682 & - & - & $?$ & $?$ & \\
\hline 7.470 & 7.475 & 7.474 & $7.473[6]$ & $\operatorname{MgXI}\left(1 s^{2}{ }^{1} S-1 s 4 p^{1} P\right)$ & \\
\hline 7.440 & - & - & $?$ & $?$ & \\
\hline 7.312 & - & 7.316 & $7.310[8]$ & $\operatorname{MgxI}\left(1 s^{2}{ }^{1} S-1 s 5 p^{1} P\right)$ & \\
\hline- & - & 7.233 & $?$ & $?$ & \\
\hline 7.172 & 7.160 & 7.179 & $7.173[4]$ & $\operatorname{Alxu}\left(1 s^{2} S_{1 / 2}-2 p^{2} P_{1 / 2,3 / 2}\right)$ & \\
\hline 7.106 & 7.104 & 7.111 & $7.106[4]$ & $\operatorname{MgXII}\left(1 s^{2} S_{1 / 2}-3 p^{2} P_{1 / 2,3 / 2}\right)$ & \\
\hline - & $6.884(?)$ & - & $?$ & *SiIX & \\
\hline- & $6.846(?)$ & - & $6.84 \quad[2]$ & ${ }^{*} \operatorname{Six}\left(1 s^{2} 2 s^{2} 2 p-1 s 2 s^{2} 2 p^{2}\right)$ & \\
\hline 6.787 & 6.78 & 6.788 & $6.786[7]$ & $\begin{array}{l}\operatorname{SixII}\left(1 s^{2} 2 s^{2} S-1 s 2 s 2 p^{4} P\right) \\
\operatorname{Six}\left(1 s^{2} 2 p^{2} P-1 s 2 p^{2} P\right)\end{array}$ & \\
\hline & 6.78 & & $6.79 \quad[2]$ & $* \operatorname{SiXI}\left(1 s^{2} 2 s^{2}-1 s 2 s^{2} 2 p\right)$ & \\
\hline 6.737 & 6.737 & 6.739 & $6.739[7]$ & $\operatorname{SixIII}\left(1 s^{2}{ }^{1} S-1 s 2 s^{3} S\right)$ & \\
\hline- & 6.703 & 6.717 & $6.717[7]$ & $\operatorname{SixII}\left(1 s^{2} 2 s^{2} S-1 s 2 s 2 p^{2} P\right)$ & \\
\hline 6.690 & 6.681 & 6.684 & $6.687[3]$ & $\operatorname{SixIII}\left(1 s^{21} S-1 s 2 p^{3} P\right)$ & \\
\hline 6.650 & 6.640 & 6.649 & $6.646[3]$ & $\operatorname{SixIII}\left(1 s^{2} S-1 s 2 p^{1} P\right)$ & \\
\hline $6.58(?)$ & $6.578(?)$ & - & $6.580[1]$ & $\operatorname{MgXII}\left(1 s^{2} S_{1 / 2}-5 p^{2} P_{1 / 2,3 / 2}\right)$ & \\
\hline 6.272 & - & - & $\begin{array}{l}6.263[3] \\
6.244\end{array}$ & ${ }^{*} \operatorname{SiXIII}\left(1 s 2 p^{1} P-2 p^{21} D\right)$ & \\
\hline & & & $\begin{array}{l}6.244[3] \\
6.224[3]\end{array}$ & ${ }^{*} \mathrm{SixIII}\left(1 s 2 s^{3} S-2 s 2 p^{3} P\right)$ & \\
\hline 6.224 & & & $\begin{array}{l}6.224[3] \\
6.200[3]\end{array}$ & $\begin{array}{l}{ }^{*} \operatorname{SixIII}\left(1 s 2 s^{1} S-2 s 2 p^{1} P\right) \\
{ }^{*} \operatorname{Sixu}\left(1 s 2 p^{1} P-2 P^{2} 1 S\right)\end{array}$ & \\
\hline 6.182 & 6.182 & 6.184 & $\begin{array}{l}6.200[3] \\
6.182[4]\end{array}$ & $\begin{array}{l}{ }^{*} \operatorname{SiXIII}\left(1 s 2 p^{1} P-2 P^{2}{ }^{1} S\right) \\
\operatorname{SiXIV}\left(1 s^{2} S_{1 / 2}-2 p^{2} P_{1 / 2,3 / 2}\right)\end{array}$ & \\
\hline
\end{tabular}


Table I (continued)

\begin{tabular}{|c|c|c|c|c|}
\hline Wavelength & $(\AA)$ & {$[1,2,3]$} & $\begin{array}{l}\text { Predicted } \\
\text { Wavelength }(\AA)\end{array}$ & Identification \\
\hline 6.049 & - & - & $6.053[4]$ & $\mathrm{AlXIII}\left(1 s^{2} S_{1 / 2}-3 p^{2} P_{1 / 2,3 / 2}\right)$ \\
\hline- & $5.971(?)$ & - & $?$ & $?$ \\
\hline $5.816(?)$ & $5.725(?)$ & 5.810 & $\begin{array}{l}5.797[3] \\
5.817[3]\end{array}$ & $\begin{array}{l}{ }^{*} \operatorname{SiXII}\left(1 s^{2} 2 s^{2} S-1 s 2 s 3 p^{2} P\right) \\
{ }^{*} \operatorname{SiXII}\left(1 s^{2} 2 p^{2} P-1 s 2 p 3 p^{2} S\right) \\
{ }^{*} \operatorname{SiXII}\left(1 s^{2} 2 p^{2} P-1 s 2 p 3 p^{2} D\right)\end{array}$ \\
\hline 5.680 & 5.672 & 5.68 & $5.681[6]$ & $\operatorname{SiXIII}\left(1 s^{2}{ }^{1} S-1 s 3 p^{1} P\right)$ \\
\hline 5.410 & 5.410 & 5.401 & $5.405[6]$ & $\operatorname{SixIII}\left(1 s^{21} S-1 s 4 p^{1} P\right)$ \\
\hline 5.283 & - & - & $5.28 \quad[1]$ & $\operatorname{SixIII}\left(1 s^{21} S-1 s 5 p^{1} P\right)$ \\
\hline 5.221 & 5.223 & 5.219 & $5.217[4]$ & $\operatorname{SiXIV}\left(1 s^{2} S_{1 / 2}-3 p^{2} P_{1 / 2,3 / 2}\right)$ \\
\hline- & 5.18 & - & $5.18 \quad[2]$ & $\begin{array}{l}{ }^{*} \mathrm{SXII}\left(1 s^{2} 2 s^{2} 2 p-1 s 2 s^{2} 2 p^{2}\right) \\
\quad \mathrm{S} \operatorname{XIV}\left(1 s^{2} 2 s^{2} S-1 s 2 s 2 p^{4} P\right)\end{array}$ \\
\hline 5.137 & 5.134 & - & $5.14[7]$ & $\begin{array}{l}\operatorname{SXIV}\left(1 s^{2} 2 p^{2} P-1 s 2 p^{4} P\right) \\
{ }^{*} \operatorname{SXIII}\left(1 s^{2} 2 s^{2}-1 s 2 s^{2} 2 p\right)\end{array}$ \\
\hline 5.099 & 5.105 & 5.100 & $5.100[7]$ & $\operatorname{Sxv}\left(1 s^{21} S-1 s 2 s^{3} S\right)$ \\
\hline- & 5.094 & 5.083 & $5.083[3]$ & $\operatorname{SxIV}\left(1 s^{2} 2 s^{2} S-1 s 2 s 2 p^{2} P\right)$ \\
\hline 5.067 & 5.068 & 5.065 & $5.065[3]$ & $\operatorname{Sxv}\left(1 s^{21} S-1 s 2 p^{3} P\right)$ \\
\hline 5.039 & 5.044 & 5.039 & $5.036[3]$ & $\operatorname{Sxv}\left(1 s^{2}{ }^{1} S-1 s 2 p^{1} P\right)$ \\
\hline 4.948 & 4.948 & - & $4.947[4]$ & $\operatorname{SixIV}\left(1 s^{2} S_{1 / 2}-4 p^{2} P_{1 / 2,3 / 2}\right)$ \\
\hline $4.884(?)$ & $4.879(?)$ & - & $?$ & $?$ \\
\hline 4.834 & 4.829 & - & $4.831[4]$ & $\operatorname{SixIV}\left(1 s^{2} S_{1 / 2}-5 p^{2} P_{1 / 2,3 / 2}\right)$ \\
\hline 4.769 & 4.774 & - & $4.770[4]$ & $\operatorname{SixIV}\left(1 s^{2} S_{1 / 2}-6 p^{2} P_{1 / 2,3 / 2}\right)$ \\
\hline 4.729 & 4.733 & 4.734 & $4.729[4]$ & $\operatorname{SxVI}\left(1 s^{2} S_{1 / 2}-2 p^{2} P_{1 / 2,3 / 2}\right)$ \\
\hline 4.674(?) & 4.685 & - & $?$ & $?$ \\
\hline- & 4.490 & - & $?$ & $* \mathrm{~S} \times I I$ \\
\hline- & 4.454 & - & $4.45[2]$ & $* \mathrm{SXIII}\left(1 s^{2} 2 s^{2}-1 s 2 s^{2} 3 p\right)$ \\
\hline 4.394(?) & 4.383 & - & $4.38 \quad[2]$ & ${ }^{*} \operatorname{SxIV}\left(1 s^{2} 2 s^{2} S-1 s 2 s 3 p^{2} P\right)$ \\
\hline 4.299 & 4.306 & - & $4.30 \quad[2]$ & $\mathrm{Sxv}\left(1 s^{21} S-1 s 3 p^{1 P}\right)$ \\
\hline $4.244(?)$ & - & - & $?$ & $?$ \\
\hline $4.184(?)$ & - & - & $?$ & $?$ \\
\hline 4.104 & 4.096 & - & $4.089[6]$ & $\operatorname{Sxv}\left(1 s^{2}{ }^{1} S-1 s 4 p^{1} P\right)$ \\
\hline- & 4.058 & - & $4.04[2]$ & $\begin{array}{l}{ }^{*} \operatorname{ArXIV}\left(1 s^{2} 2 s^{2} 2 p-1 s 2 s^{2} 2 p^{2}\right) \\
\operatorname{ArXVI}\left(1 s^{2} 2 s^{2} S-1 s 2 s 2 p^{4} P\right)\end{array}$ \\
\hline- & $4.014(?)$ & - & $4.01[2]$ & $\begin{array}{l}\operatorname{ArXVI}\left(1 s^{2} 2 p^{2} P-1 s 2 p^{2}{ }^{4} P\right) \\
{ }^{*} \operatorname{ArXv}\left(1 s^{2} 2 s^{2}-1 s 2 s^{2} 2 p\right)\end{array}$ \\
\hline 4.004 & 3.995 & - & 3.99 & $\operatorname{ArXvIr}\left(1 s^{2}{ }^{1} S-1 s 2 s^{3} S\right)$ \\
\hline blend & 3.969 & - & $3.97[2]$ & $\operatorname{ArXvu}\left(1 s^{2}{ }^{1} S-1 s 2 p^{3} P\right)$ \\
\hline 3.949 & 3.950 & - & $3.965[9]$ & $\operatorname{ArXVII}\left(1 s^{21} S-1 s 2 p^{1} P\right)$ \\
\hline 3.786 & - & - & $3.784[4]$ & $\operatorname{SxVI}\left(1 s^{2} S_{1 / 2}-4 p^{2} P_{1 / 2,3 / 2}\right)$ \\
\hline 3.733 & 3.736 & - & $3.733[4]$ & $\operatorname{Arxvin}\left(1 s^{2} S_{1 / 2}-2 p^{2} P_{1 / 2,3 / 2}\right)$ \\
\hline $3.698(?)$ & - & - & $3.696[4]$ & $\operatorname{SXVI}\left(1 s^{2} S_{1 / 2}-5 p^{2} P_{1 / 2,3 / 2}\right)$ \\
\hline 3.651 & - & - & $3.650[4]$ & $\mathrm{SxVI}\left(1 s^{2} S_{1 / 2}-6 p^{2} P_{1 / 2,3 / 2}\right)$ \\
\hline 3.573 & 3.572 & - & $3.572[7]$ & $\mathrm{KXVIII}\left(1 s^{2}{ }^{1} S-1 s 2 s^{3} S\right)$ \\
\hline 3.550 & - & - & & $\mathrm{KXVIII}\left(1 s^{2}{ }^{1} S-1 s 2 p^{3} P\right)$ \\
\hline 3.534 & 3.542 & - & $3.534[6]$ & $\mathrm{KXVIII}\left(1 s^{2}{ }^{1} S-1 s 2 p^{1} P\right)$ \\
\hline $3.445(?)$ & 3.436 & - & $3.45[2]$ & ${ }^{*} \operatorname{ArXv}\left(1 s^{2} 2 s^{2}-1 s 2 s^{2} 3 p\right)$ \\
\hline- & 3.392 & - & $3.40 \quad[2]$ & ${ }^{*} \mathrm{ArXvI}\left(1 s^{2} 2 s^{2} S-1 s 2 s 3 p^{2} P\right)$ \\
\hline- & 3.374 & - & $?$ & $*$ ArXVI \\
\hline 3.371 & 3.349 & - & $3.367[2]$ & $\operatorname{ArXVII}\left(1 s^{2}{ }^{1} S-1 s 3 p^{1 P}\right)$ \\
\hline- & $3.238(?)$ & - & $3.254[5]$ & $\begin{array}{l}{ }^{*} \operatorname{Caxvi}\left(1 s^{2} 2 s^{2} 2 p-1 s 2 s^{2} 2 p^{2}\right) \\
\quad \text { CaxviII }\left(1 s^{2} 2 s^{2} S-1 s 2 s 2 p^{4} P\right)\end{array}$ \\
\hline 3.225 & $3.219(?)$ & - & $3.22 \quad[7]$ & $\begin{array}{l}\mathrm{Caxvir}\left(1 s^{2} 2 p^{2} P-1 s 2 p^{24} P\right) \\
{ }^{*} \mathrm{CaxvII}\left(1 s^{2} 2 s^{2}-1 s 2 s^{2} 2 p\right)\end{array}$ \\
\hline
\end{tabular}


Table I (continued)

\begin{tabular}{|c|c|c|c|c|}
\hline Wavelength & $(\AA)$ & {$[1,2,3]$} & $\begin{array}{l}\text { Predicted } \\
\text { Wavelength }(\AA)\end{array}$ & Identification \\
\hline 3.206 & 3.207 & - & $3.21 \quad[7]$ & $\operatorname{CaxIX}\left(1 s^{2}{ }^{1} S-1 s 2 s^{3} S\right)$ \\
\hline 3.197 & blend & - & $3.20 \quad[7]$ & $\operatorname{CaxvIII}\left(1 s^{2} 2 s^{2} S-1 s 2 s^{2} p^{2} P\right)$ \\
\hline 3.186 & 3.187 & - & $3.19[2]$ & $\mathrm{CaxIX}\left(1 s^{21} S-1 s 2 p^{3} P\right)$ \\
\hline 3.173 & 3.174 & - & $3.174[2]$ & $\operatorname{CaxIx}\left(1 s^{21} S-1 s 2 p^{1} P\right)$ \\
\hline 3.16 & - & - & $3.151[4]$ & $\operatorname{ArXVIII}\left(1 s^{2} S_{1 / 2}-3 p^{2} P_{1 / 2,3 / 2}\right)$ \\
\hline 3.026 & 3.022 & - & $3.020[4]$ & $\operatorname{Caxx}\left(1 s^{2} S_{1 / 2}-2 p^{2} P_{1 / 2,3 / 2}\right)$ \\
\hline- & $2.990(?)$ & - & $2.987[4]$ & $\operatorname{ArXviII}\left(1 s^{2} S_{1 / 2}-4 p^{2} P_{1 / 2,3 / 2}\right)$ \\
\hline 2.750 & 2.748 & - & $2.75[1]$ & ${ }^{*} \mathrm{CaXVIII}\left(1 s^{2} 2 s^{2} S-1 s 2 s 3 p^{2} P\right)$ \\
\hline 2.706 & 2.703 & - & $2.71[2]$ & $\operatorname{CaxIx}\left(1 s^{21} S-1 s 3 p^{1} P\right)$ \\
\hline 2.64 & $\begin{array}{l}2.634 \\
2.621\end{array}$ & - & $2.619[5]$ & TixxI + innershell \\
\hline 2.61 & 2.608 & & & \\
\hline 2.573 & 2.572 & - & $2.58 \quad[2]$ & $\operatorname{CaxIx}\left(1 s^{21} S-1 s 4 p^{1} P\right)$ \\
\hline 2.55 & $2.548(?)$ & - & $2.549[4]$ & $\operatorname{Caxx}\left(1 s^{2} S_{1 / 2}-3 p^{2} P_{1 / 2,3 / 2}\right)$ \\
\hline 2.516 & $2.508(?)$ & - & $2.52[1]$ & $\operatorname{CaxIX}\left(1 s^{2}{ }^{1} S-1 s 5 p^{1} P\right)$ \\
\hline 2.42 & 2.441 & - & $?$ & ? \\
\hline 2.215 & 2.196 & - & $2.189[5]$ & CrXXIII + innershell \\
\hline 2.175 & 2.179 & & & \\
\hline$\sim 2.01$ & 2.009 & - & $2.011[5]$ & MnXxiv + innershell \\
\hline 1.94 & 1.931 & - & $1.937[5]$ & FeII \\
\hline 1.883 & 1.885 & - & $1.886[5]$ & $\begin{array}{l}{ }^{*} \mathrm{FexXII}\left(1 s^{2} 2 s^{2} 2 p-1 s 2 s^{2} 2 p^{2}\right), \text { FexXIV? } \\
{ }^{*} \mathrm{FexXIV}\left(1 s^{2} 2 s^{2} S-1 s 2 s^{2} 2 p^{4} P\right)\end{array}$ \\
\hline$\sim 1.874$ & 1.875 & - & $1.872[7]$ & ${ }^{*} \mathrm{FexXIV}\left(1 s^{2} 2 p^{2} P-1 s 2 p^{2}{ }^{4} P\right)$ \\
\hline$\sim 1.874$ & 1.868 & - & $1.870[7]$ & ${ }^{*} \mathrm{FexxIII}\left(1 s^{2} 2 s^{2}-1 s 2 s^{2} 2 p\right)$, FexxIV? \\
\hline$\sim 1.867$ & 1.865 & - & $1.862[7]$ & $\operatorname{Fexxv}\left(1 s^{2}{ }^{1} S-1 s 2 s^{3} S\right)$ \\
\hline 1.862 & 1.860 & - & $1.860[7]$ & $\operatorname{FexxIV}\left(1 s^{2} 2 s^{2} S-1 s 2 s 2 p^{2} P\right)$ \\
\hline 1.857 & 1.855 & - & $1.856[7]$ & $\operatorname{Fexxv}\left(1 s^{2}{ }^{1} S-1 s 2 p^{3} P\right)$ \\
\hline 1.852 & 1.850 & - & $1.850[2]$ & $\mathrm{Fexxv}\left(1 s^{2+} S-1 s 2 p^{1} P\right)$ \\
\hline $1.79(?)$ & 1.780 & - & $1.79[5]$ & FexxvI $\left(1 s^{2} S_{1 / 2}-2 p^{2} P_{1 / 2,3 / 2}\right)$ \\
\hline 1.62 & 1.587 & - & $1.593[5]$ & NixxviI + innershell \\
\hline 1.59 & & & & \\
\hline 1.58 & 1.567 & - & 1.58 & $\operatorname{Fexxv}\left(1 s^{21} S-1 s 3 p^{1} P\right)$ \\
\hline$\sim 1.545(?)$ & 1.544 & - & $1.55 \quad[1]$ & ${ }^{3}$ NixXVIII $\left(1 s^{2} S-2 p^{2} P\right)$ \\
\hline$\sim 1.52(?)$ & 1.509 & - & 1.50 & $\operatorname{Fexxv}\left(1 s^{21} S-1 s 4 p^{1} P\right)$ \\
\hline- & 1.486 & - & $1.486[6]$ & Cuxxvin + - innershell \\
\hline$\sim 1.47(?)$ & 1.456 & - & $1.470[1]$ & $\operatorname{Fexxv}\left(1 s^{21} S-1 s 5 p^{1} P\right)$ \\
\hline
\end{tabular}

\section{References}

[1] Naval Research Laboratory: Meekins et al. (1970); Doschek et al. (1971a, b)

[2] Goddard Space Flight Center: Neupert (1971a)

[3] Aerospace Corporation: Walker and Rugge (1970, 1971)

[4] Garcia and Mack (1965)

[5] House (1969)

[6] Blake (1968a)

[7] Gabriel and Jordan (1969a), wavelengths extrapolated from [7].

[8] Flamberg (1942)

[9] Cohen et al. (1968)

[10] Cowan (1971)

* Identification uncertain. 
(3) Consistency with available laboratory observations.

(4) Observation of several lines in each series. The actual identification procedures may be found in the references cited at the end of the table.

The strongest lines in Table I are the resonance lines of hydrogenic and helium-like ions. The Lyman series up to Lyman- $\varepsilon$ has been identified for silicon and sulfur. Also, many of the transitions listed in the table are innershell transitions, i.e., the transition involves an electron in the $1 s$ or K-shell. The inner-shell transitions are weak compared to the resonance lines except in the case of iron. For iron, emission due to Fexxiv, and possibly FexxII and FexxIII, is about equal to emission from Fexxy. Presumably, a similar percentage would be found for manganese, chromium, and nickel if these elements were sufficiently abundant in the Sun to permit accurate wavelength and intensity measurements.

Figure 13 shows the NRL LiF and EDDT spectra for a very intense flare that occurred on November 16, 1970. Inspection of these spectra will show most of the lines listed in Table I. Many of the lines given in the table are seen only in spectra of the most intense flares. However, some of the lines are more prominent in spectra of cooler flares because they are produced by stages of ionization formed at lower temperatures. It is instructive to compare Figures 9 and 13. The very weak emission teatures in Table I are only seen clearly in the November 16 event.

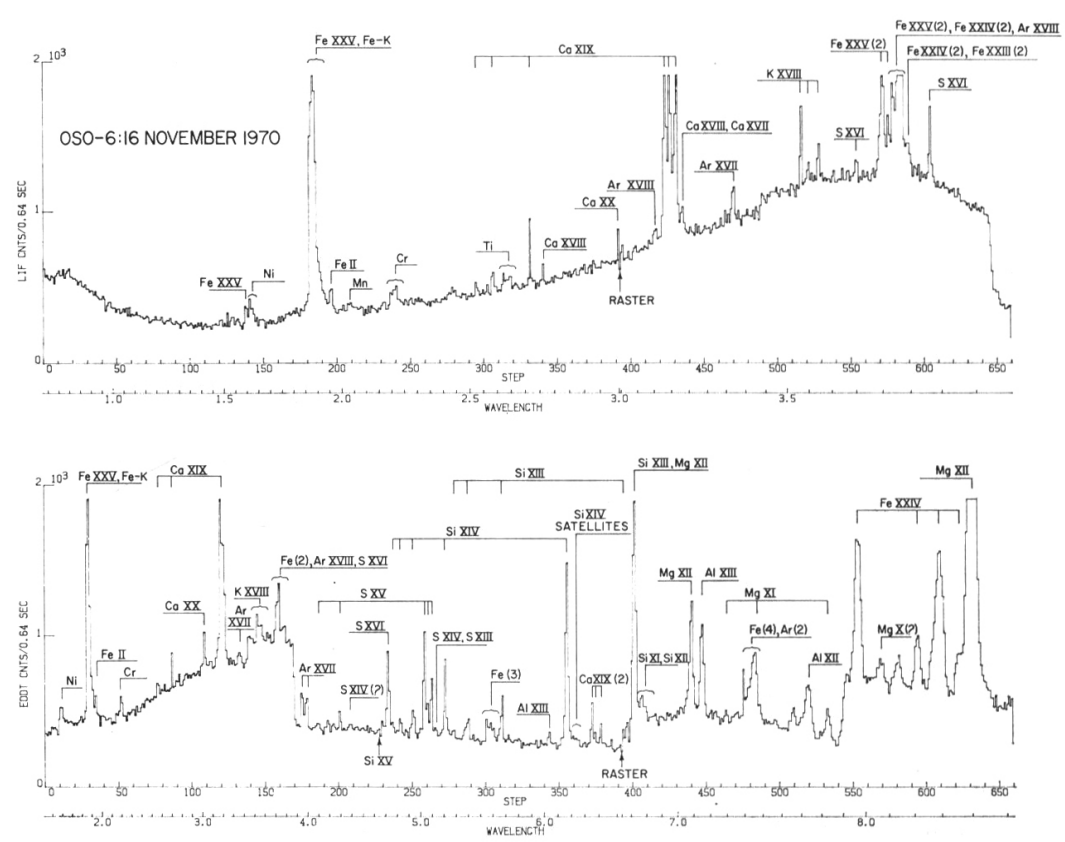

Fig. 13. Uncorrected NRL spectra of the intense soft X-ray flare of November 16, 1970. Many of the emission lines listed in Table 1 are seen in these spectra. Note in particular, the suspected satellite lines to the SixIV ion near $\sim 6.2 \AA$. Also note the line at $2.75 \AA$, tentatively identified as an innershell transition in CaxviIr. Compare with Figures 7 and 9. The continuum emission is strongly influenced by temporal effects. The numbers in parentheses beside some of the transitions indicate spectral order. 
The LiF spectrum of Figure 13 shows emission features due to nickel, chromium, and titanium. Higher members of the resonance line series of calcium, argon, and sulfur can also be identified, but the highest members of these series are only marginally detected. Emission near the wavelength of the Lyman- $\alpha$ line of FexXVI is indicated, but this line is never a strong feature in flare spectra observed to date. The low intensity of this line allows an upper limit to be estimated for the temperature of the flare plasma if thermal equilibrium is assumed.

The Lyman- $\alpha$ Caxx line in Figure 13 is distorted due to a raster maneuver of the spacecraft as explained in Section $2 B$. Also note that the iron-line blend $\sim 1.9 \AA$, the Caxix lines $\sim 3.2 \AA$, and the feature $\sim 3.7 \AA$ are saturated. The relative strengths of these features may be estimated from the EDDT spectrum, however.

Other emission lines of interest in the LiF spectrum, which will subsequently be discussed, are the Fe II lines $\sim 1.932 \AA$, the weak CaxvII and CaxvIII satellite lines found near the Caxix forbidden line $(\sim 3.22 \AA)$, and the KxviII resonance, intercombination, and forbidden lines $\sim 3.55 \AA$. The intercombination line of KXVIII has not been previously reported because of its low intensity.

The EDDT spectrum of Figure 13 reveals many lines in the resonance line series of Mgxi, Mgxir, AlxII, AlxIII, SixIII, SixIV, Sxv, SxVI, and ArXVII, and weaker features identified as satellites of hydrogenic and helium-like ions. Substantial emission due to second, third, and fourth order diffraction of the $1.9 \AA$ iron-line blend, and second order diffraction of the Caxix lines are also apparent in this spectrum. The resonance, intercombination, and forbidden lines of CaXIX are clearly resolved in second order.

A comparison of the intensity of the Lyman- $\alpha$ lines of hydrogenic ions to the corresponding intensity of the resonance lines of the helium-like ions for the same elements in Figure 13 (and also in Figures 7, 8, 9 and 10) shows that the hydrogen-like line strength decreases relative to the helium-like resonance line strength with increasing atomic number. This observation is qualitatively expected because the heavier elements require more energy for ionization. The Caxx Lyman- $\alpha$ line, while easily identifiable in the spectra of many flares observed to date, never approaches the intensity of the CaxIX resonance line, as is the case for sulfur. From observations such as these, the electron temperatures in flare plasmas that produce the soft $X$-ray emission can be estimated to be $\sim 15-40 \times 10^{6} \mathrm{~K}$ (see Section 4). Finally, we wish to emphasize that the November 16, 1970 flare was very impulsive, and therefore the shape of the continuum is strongly influenced by temporal effects.

Two other line groups in Table I require more detailed discussion. First, the short wavelength part of Table I shows many iron ion transitions near $\sim 1.9 \AA$. It is clear from the low resolution of the LiF crystal near $\sim 1.9 \AA$ in Figures 7, 9, and 13 that these features cannot be resolved in first order. Neupert and Swartz (1970), however, resolved the iron-line blend in the third and fourth orders of diffraction in their OSO-5 ADP spectra. Their data for a large flare are shown in Figure 14, and from an analysis of these spectra they derived the wavelengths listed in Table I.

Subsequently, Doschek et al. (1971a) resolved the iron-line blend in second order (LiF spectrometer). Their data are shown in Figure 15 with a recent laboratory spec- 
trum obtained by Lie and Elton (1971). Table II lists the most important transitions believed to produce the blended first and second order features near $\sim 3.75 \AA$ in the NRL spectra. The numbers above the emission features correspond to the numbered transitions in Table II. The flare spectrum shown is an amplification of the data in Figure 9.

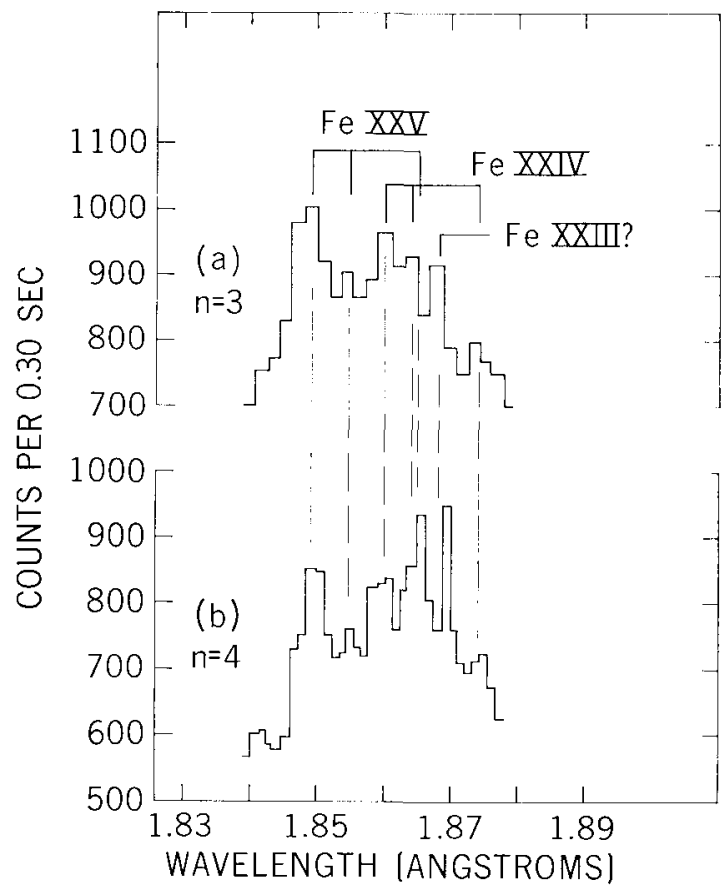

Fig. 14. Third and fourth order spectra of iron-line emission $\sim 1.86 \AA$. Emission due to Fexxv, FexxIv and possibly FexxiII is clearly resolved. Compare with Figure 15. Spectra courtesy of W. M. Neupert, Goddard Space Flight Center.

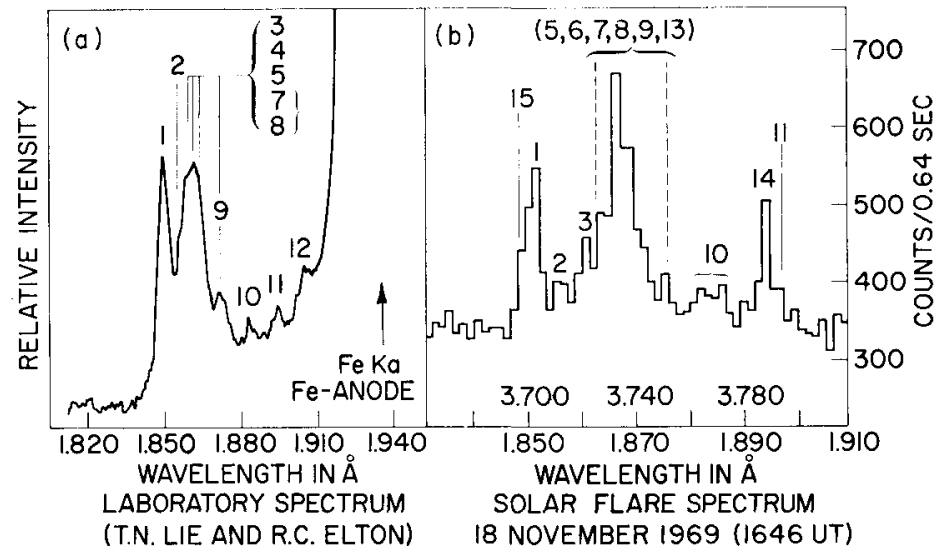

Fig. 15. An NRL second order spectrum of iron-line emission $\sim 1.9 \AA$ compared to a recent laboratory spectrum. The numbers above the features correspond to the transitions identified by the same numbers in Table II. The solar wavelength scale gives first and second order wavelengths. 
TABLE $1 \mathrm{I}$

Emission line identifications

No. Ion and transition

\begin{tabular}{llll}
$\begin{array}{l}{[1]} \\
\begin{array}{l}\text { Laboratory } \\
\lambda(\AA)\end{array}\end{array}$ & OSO $5 \lambda(\AA)$ & $\begin{array}{l}{[3],[4],[5]} \\
\text { Theory } \lambda(\AA)\end{array}$ & NRL $\lambda(\AA)$ \\
\hline & & & \\
1.851 & 1.850 & $1.855[3]$ & 1.852 \\
1.856 & 1.855 & $1.860[4]$ & 1.857 \\
1.861 & 1.860 & $1.866[4]$ & 1.862 \\
- & - & $1.868[4]$ & - \\
- & 1.865 & $1.869[4]$ & $\sim 1.867$ \\
- & 1.865 & $1.869[4]$ & $\sim 1.867$ \\
- & 1.875 & $1.876[4]$ & $\sim 1.874$ \\
- & 1.875 & $1.876[4]$ & $\sim 1.874$ \\
- & 1.868 & $1.875[3]$ & $\sim 1.874$ \\
1.872 & - & $1.886[3]$ & $1.881-1.885$ \\
1.886 & - & $1.897[3]$ & $\sim 1.896$ \\
1.896 & - & $1.907[3]$ & - \\
1.906 & - & $3.7329[5]$ & 3.735 \\
- & - & $3.7845[5]$ & 3.786 \\
- & - & $3.6959[5]$ & 3.698 \\
- & - & $3.6495[5]$ & 3.651 \\
- & - & &
\end{tabular}

\section{References}

[1] Lie and Elton (1971)

[2] Neupert and Swartz (1970)

[3] House (1969)

[4] Gabriel and Jordan (1969b), wavelengths extrapolated from [4].

[5] Garcia and Mack (1965)

There is a close similarity in appearance between the laboratory spectrum and the flare spectrum in Figure 15, although there is a difference between the spectra. The laboratory electron temperature was similar to the flare temperature $\left(\sim 30 \times 10^{6} \mathrm{~K}\right)$, but the electron density was $\approx 10^{20} \mathrm{~cm}^{-3}$. Therefore, transition number 6 in Table II which is observed in flare spectra for the helium-like ions of lighter elements than iron, and is also expected to be present for iron, is not present in the laboratory spectrum because of collisional quenching of the $2{ }^{3} S$ state.

Finally, Mandelshtam and his colleagues at the Lebedev Institute in Moscow have resolved the iron-line blend $\sim 1.9 \AA$ using a quartz crystal on the U.S.S.R. Intercosmos-4 spacecraft (Vasiljev et al., 1971). Their data show a large number of K-lines due to Fexxiv and possibly Fexxin, and lines due to Fexxv. From the intensity ratio of Fexxiv to Fexxv, and from the Doppler width of the resonance line of Fexxv, they determined electron and ion temperatures of $20 \times 10^{6} \mathrm{~K}$ and $30 \times 10^{6} \mathrm{~K}$, respectively, for the November 16 event shown in Figure 13.

One of the interesting features of their spectra is the weakness of the Fexxv intercombination line. The NRL data of Figure 13 are from the same flare, but the NRL observations are not exactly coincident in time with the Russian observations ( $\sim 1$ min which may be important because of the impulsive nature of this event), and 
the second order Fexxv resonance line in the NRL spectrum is saturated. Subsequent NRL scans show a resonance to intercombination line ratio which appears to be somewhat smaller than is indicated by the Intercosmos data $(\approx 3: 1)$. Most of the NRL second order iron-line spectra obtained from a number of flares indicate a ratio of $\sim 2: 1$ for the Fexxv resonance to intercombination intensity ratio. Perhaps the emission in the NRL data identified as the intercombination line is actually a blend of the intercombination line and nearby satellite lines.

Two remaining aspects of the iron-line emission near $\sim 1.9 \AA$ are now discussed. Firstly, Figures 9 and 13 show a statistically significant emission feature near $1.932 \AA$. The wavelength difference between this feature and the peak of the Fexxv resonance line at $1.850 \AA$ agrees closely with the theoretically calculated (House, 1969) wavelength difference between Fexxv $\left(1 s^{2}{ }^{1} S-1 s 2 p^{1} P\right)$ and the $\mathrm{K} \alpha$ line of Fe II. This feature is present in the $\mathrm{X}$-ray spectra of disk flares.

The large event of August 12, 1970 (see Figures 36 and 35e, which will be discussed in Section 5) was a limb flare and in this event the $1.932 \AA$ feature is definitely absent. Neupert et al. (1969) have also discussed this feature and suggest that either non-thermal electron excitation in a cooler gas or fluorescence radiation from the lower chromosphere is responsible for the emission. Because the feature is not seen in limb flares but is seen in disk flares (see Figures 9 and 13; the November 16, 1970 event and November 18, 1969 event are disk flares), we conclude that the fluorescence mechanism is the more likely explanation (unless a sufficient flux of electrons from the flare region can reach photospheric layers), and that the chromosphere or photosphere is similar to a Lambertian radiator. Thus for limb flares, the radiation observed is zero. From this observation the photospheric abundance of iron may be inferred (Tomblin, 1971a) if the fluorescence mechanism is assumed; this problem is under investigation.

Secondly, Tomblin (1971b) has suggested that a small amount of Compton backscattering off the solar photosphere may produce measurable emission on the long wavelength side of the Fexxv group. We also calculate that such emission should be observable. Tomblin finds that the spectral shape of the backscattered radiation depends on the location of the flare above the solar disk. The total amount of backscattered radiation is predicted to be $\sim 10 \%$ of the Fexxv group. A thorough discussion of this problem is in preparation (Tomblin, 1971a).

Another group of lines of interest in Table I are the lines near $8.3 \AA$. Some of these lines are quite weak and can only be established with confidence by summing several spectral scans. The weakest lines in this group have not been reported previously. Figure 16 shows the result of summing three spectral scans available from an intense event on August 12, 1970. Lines listed in Table I at $8.396 \AA$ and $8.458 \AA$ cannot be clearly seen in the sum because of the large intensity of the Lyman- $\alpha$ MgXII line $(8.421 \AA)$ during this event. However, these lines are present in less intense flares observed by NRL. The numbers over the emission features correspond to the numbers in parentheses given to the right of the corresponding transitions in Table I.

The NRL group proposes that some of these lines are due to Fexxiv. The wavelengths of the FexxIV transitions listed in Table I have been calculeted by Cowan 


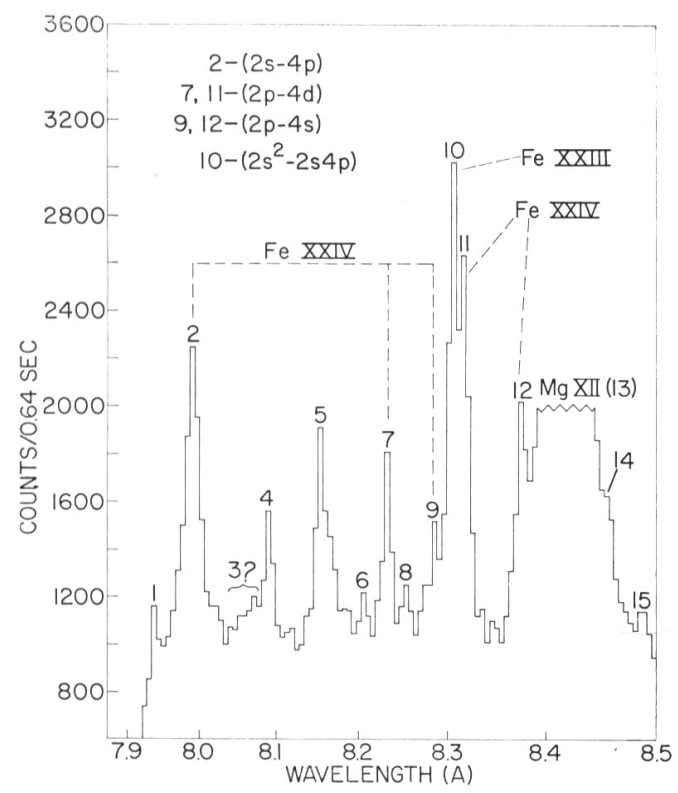

Fig. 16. Summed EDDT spectra near $\sim 8 \AA$ for the flare of August 12, 1970. Lines identified as due to Fexxuv are indicated. The numbers above the emission features correspond to the numbered transitions in Table I. Note the very weak unidentified lines (numbers 6 and 8) and the two groups (?) of stronger lines (numbers 3, 4 and 5). The identification of the stronger lines is also uncertain.

(1971) with a Hartree-Fock computer code using intermediate coupling. The $0.2 \%$ disagreement in wavelength with the Hartree-Fock calculations is considered insignificant (Cowan, 197I). The proposed identifications are based on the following:

(1) Inner-shell transitions of Fexxiv are observed in flare spectra (see Figures 14 and 15), so optical transitions are expected.

(2) Hartree-Fock calculations give wavelengths which agree to within $0.2 \%$ with measured wavelengths.

(3) During the decay phase of the August 12 event (cooling period), the lines identified as Fexxiv lines in Table I decreased in flux by approximately the same amount as the Lyman- $\alpha$ Sxvi line and other lines of highly ionized elements, while the flux in the other prominent lines in this region, e.g., lines at $8.15 \AA$ and $8.06 \AA$, either decreased or increased only slightly in intensity.

(4) The relative intensities of the Fexxiv lines are in general agreement with theoretical expectations. For example, the ratio of the FexxIV $\left(1 s^{2} 2 p^{2} P_{1 / 2,3 / 2}-1 s^{2} 4 s^{2} S_{1 / 2}\right)$ lines and the ratio of the FexxIV $\left(1 s^{2} 2 p^{2} P_{3 / 2}-1 s^{2} 4 d^{2} D_{5 / 2}\right)$ line to the FexXIV $\left(1 s^{2} 2 p\right.$ ${ }^{2} P_{1 / 2}-1 s^{2} 4 d^{2} D_{3 / 2}$ ) line are $\sim 2: 1$, in agreement with expectations based on statistical weights.

These identifications of Fexxiv transitions appear to be definite, although blending of these lines with other weak lines in this spectral region cannot be dismissed at present. Also note the line at $8.307 \AA$ that we tentatively identify as FexxIII. The identifi- 
cation is based on Hartree-Fock calculations by Cowan (1971). A more complete publication on this subject is in preparation by NRL.

Finally, before leaving the subject of line identifications, we comment on the absolute wavelengths given by the authors in Table I. The crystal spectrometers flown on satellites are calibrated before flight. Nevertheless, Table I shows some spread in the absolute wavelengths listed by the three groups. The methods of calibration may be found in the references at the end of the table. We comment here on the NRL wavelengths, only. Our experimental absolute wavelengths are presently not better than $\sim 0.02 \AA$ at the short wavelength end of the LiF and EDDT spectra, and not better than $\sim 0.005 \AA$ at the long wavelength end due to a small misalignment of the instruments in the spacecraft. In the middle of the spectra, the error lies somewhere between these extremes. Relative wavelengths, however, are more accurate, provided the lines compared are not separated by a large difference in wavelength. It is anticipated that better absolute wavelengths will become available.

\section{Spectral Characteristics}

\section{A. RESONANCE LINES}

As mentioned in Section 3B, the strongest lines seen in flare spectra are the resonance lines of hydrogenic and helium-like ions. This observation is a consequence of the ionization balance in the flare plasma. For an optically thin, high temperature plasma in ionization equilibrium, the relative ion abundances are determined by the equations (Woolley and Allen, 1948),

$$
N_{\mathrm{e}} N_{z} Q_{z}=N_{\mathrm{e}} N_{z+1}\left(\alpha_{z+1}^{r}+\alpha_{z+1}^{d}\right),
$$

which relate the number densities of ions of net charge $z$ and $z+1$. In Equations (3) $N_{\mathrm{e}}$ is the electron number density, $N_{z}$ is the ion number density of an ion with net charge $z, Q_{z}$ is the electron ionization coefficient $\left(\mathrm{cm}^{3} \mathrm{~s}^{-1}\right)$, and $\alpha_{z+1}^{r}$ and $\alpha_{z+1}^{d}$ are the radiative and dielectronic recombination coefficients $\left(\mathrm{cm}^{3} \mathrm{~s}^{-1}\right)$, respectively. Burgess (1964a) has shown that dielectronic recombination is an important process in the solar corona, and under the conditions in the solar atmosphere, both photoionization and three-body recombination may be neglected (Jordan, 1969). The validity of ionization equilibrium in flares is considered in Section 5A. For the present, semi-quantitative discussion, it is assumed that at least quasi-equilibrium is valid in the solar flare plasma. It is understood that non-steady state conditions may exist in flare plasmas. Nevertheless, assumption of a multi-thermal plasma allows temperatures to be estimated which should approximate excitation conditions in different regions of the plasma.

Solutions of Equations (3) for the relative ion abundances as a function of eiectron temperature have been obtained by a number of authors (House, 1964 (without dielectronic recombination); Allen and Dupree, 1969; Jordan, 1969; Landini and Monsignori Fossi, 1970; Beigman et al., 1971a). These solutions show that relatively low temperatures are sufficient to ionize elements lighter than calcium up to the helium- 
like ionization stage ( $T \approx 8 \times 10^{6} \mathrm{~K}$ ), but higher temperatures are required to produce further appreciable ionization $\left(T \approx 15 \times 10^{6} \mathrm{~K}\right)$. This is due to the substantially larger electron energies necessary to remove an electron from the $K$ shell relative to the energies required to ionize from the $\mathrm{L}$ shell or $\mathrm{M}$ shell. The solutions also show that heliumlike ions can exist over a wide range of temperatures, and that as much as $95 \%$ of a particular element may be concentrated in the helium-like ionization stage.

These theoretical results explain qualitatively most of the features of soft X-ray line emission during flares. The dominance of resonance lines of hydrogenic and heliumlike ions in flare spectra follows directly from a high electron temperature, or a nonthermal equivalent. Line excitation in flares can be produced by electron impact excitation (from the ground state in most cases) and dielectronic and radiative recombination followed by cascades. If for simplicity, electron excitation is considered to be the most important process, then Figure 17 gives the theoretical equilibrium emission rate for a number of resonance lines of hydrogen-like and helium-like ions as a function of temperature. If the flare region is assumed to be isothermal, then the ratio of any pair of hydrogenic to helium-like resonance lines of the same element gives the temperature. Ratios of lines of different elements may also be used to determine the temperature of an isothermal plasma, but errors will be introduced due to uncertainties in the relative element abundances. The results in Figure 17 were obtained assuming the EUV coronal abundances of Pottasch (1964), and ionization equilibrium was calculated from cross-sections in Seaton (1959), Burgess and Seaton (1964), and Burgess (1965).

Figure 17 shows that the emission rate of each ion is asymmetric about the peak flux. The rapid rise in emission before peak flux is due to both an increase in the electron

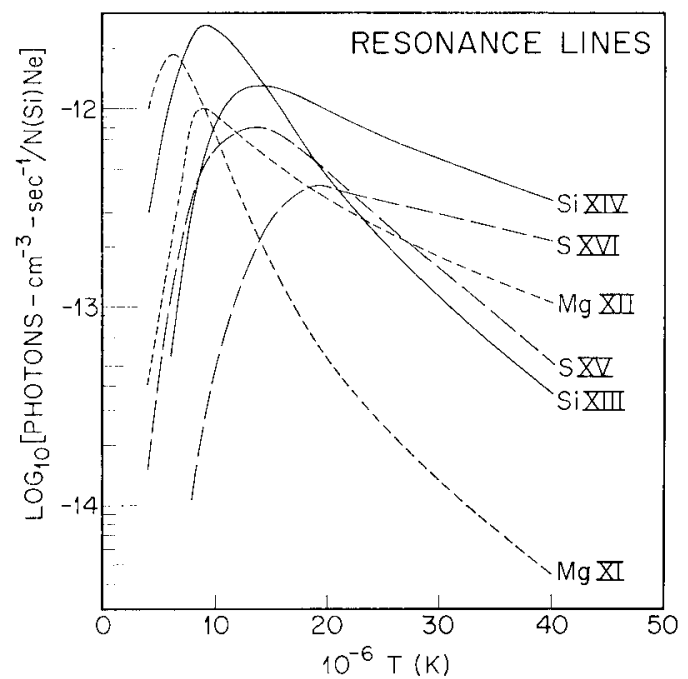

Fig. 17. Theoretical emission coefficients of abundant solar elements under the assumption of collisional excitation. The emission functions are normalized to the silicon abundance. The coronal EUV abundances of Pottasch (1964) were used. 
excitation coefficient and an increase in the percentage abundance of the ion. The decrease of emission rate after peak flux is slower because the ion abundance decreases rather slowly due to further ionization, and the electron excitation coefficient continues to increase due to the rising temperature.

Figure 18 shows the ratio of the emission rates of hydrogenic to helium-like ions as a function of temperature for several elements observed in flare spectra, under the assumption of collisional excitation and an isothermal plasma. Temperatures of $\sim 50 \times 10^{6} \mathrm{~K}$ are required before emission from the Lyman- $\alpha$ line of FexxvI is even

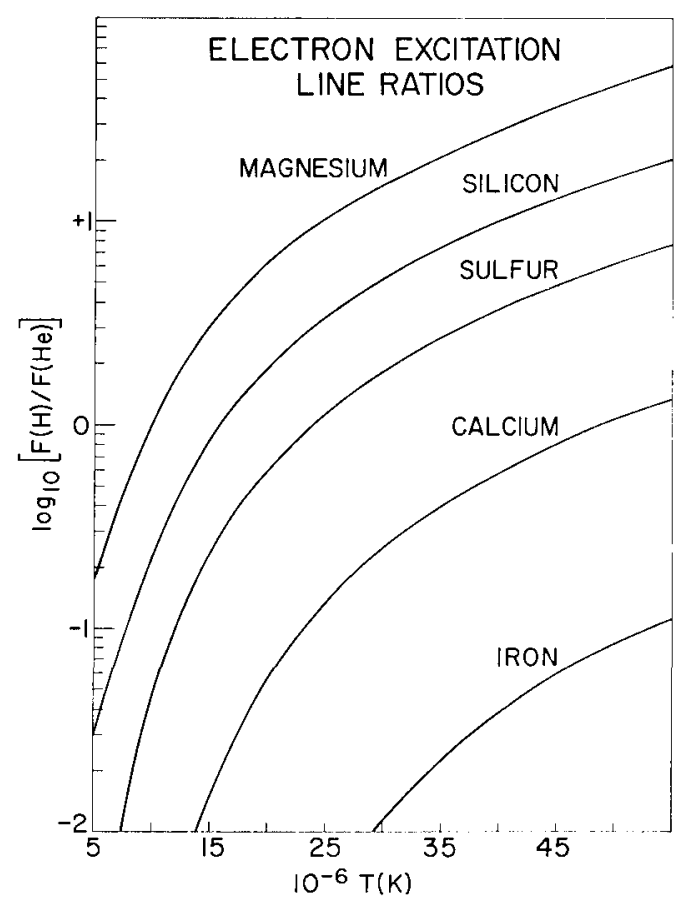

Fig. 18. The theoretical flux ratio of the resonance lines of hydrogen-like to helium-like ions for the elements indicated. Calculations assume that the lines are formed by collisional excitation.

$10 \%$ of the flux from Fexxv. Inspection of the spectra in Figures 7, 8, 9, 10, and 13 shows that, under the assumptions given above, temperatures determined from line ratios in hot flares are typically $\sim 20-30 \times 10^{6} \mathrm{~K}$. (It is feasible to qualitatively compare resonance lines of hydrogenic ions to resonance lines of heliumlike ions in these figures even though the spectra are uncorrected for instrumental effects because these lines are relatively close in wavelength and instrumental edges are not involved. The error introduced is of the same order of magnitude as the errors due to present uncertainties in the ionization and recombination cross-sections.)

The above spectra also show, however, that, as expected, the assumption of an isothermal plasma is not valid. For instance, in Figures 9 and 10 of the November 18 , 
1969 event, the calcium line ratio $(\mathrm{LiF})$ indicates $T_{\mathrm{e}} \approx 30 \times 10^{6} \mathrm{~K}$, while the magnesium line ratio (KAP) gives $T_{\mathrm{e}} \approx 10 \times 10^{6} \mathrm{~K}$. It is assumed for the KAP spectrum that $\sim 50$ percent of the emission blend $\sim 9.17 \AA$ is due to the resonance line of $\mathrm{MgXI}$, and that the other $50 \%$ is primarily due to the intercombination and forbidden line of $\mathrm{MgxI}$. This assumption is consistent withboth observation and theory of the helium-like ions (Freeman et al., 1971). A detailed picture of the plasma temperature and emission measure structure, however, will require improvements in theoretical excitation, ionization, and recombination cross-sections and accurate corrections for instrumental efficiencies.

\section{B. HELIUM-LIKE IONS AND SATELLITE LINES}

While the hydrogenic and helium-like resonance line series dominate the soft X-ray spectrum below $8.5 \AA$, Table I reveals a large number of lines identified as inner-shell transitions in $\mathrm{Mg}, \mathrm{Si}, \mathrm{S}, \mathrm{Ar}, \mathrm{Ca}$, and $\mathrm{Fe}$. Also, the intercombination and forbidden lines of helium-like ions are quite prominent. The identification of the helium-like lines and the satellite lines is discussed below. A discussion of intensity variations of these features during flares is deferred to Section 5.

The forbidden lines of helium-like ions were first identified in solar spectra by Gabriel and Jordan (1969a, b) from a comparison of laboratory and solar data. Gabriel and Jordan also observed a number of inner-shell transitions in laboratory data which they identified as lithium-like satellite lines of helium-like ions. These lines were produced in a theta-pinch plasma $\left(N_{\mathrm{e}} \approx 10^{16} \mathrm{~cm}^{-3}\right)$. By observing the time-histories of the satellite lines, Gabriel and Jordan were able to demonstrate that most of the lines were formed by dielectronic recombination to the helium-like ions. The observed lines that cannot be formed by dielectronic recombination only appeared at higher electron densities; conditions under which collisional excitation of the lithiumlike ions may become important.

In a further communication, Gabriel and Jordan (1969b) developed a theory based on the helium-like ions for deriving electron densities in active regions. The theory may also be applicable to flare plasmas. Briefly, Gabriel and Jordan assume that the helium-like ions are excited by collisional excitation at a temperature that corresponds to the maximum emission rate of the ions (see Figure 17). The $2{ }^{3} S$ state is sufficiently long-lived for electron excitation from the $2{ }^{3} S$ state into the $2{ }^{3} P$ state to be important, and therefore the intensity ratio of the forbidden line to the intercombination line is density dependent. Under assumptions regarding the relative excitation rates from the ground state into the $2^{3} S$ and $2{ }^{3} P$ levels, and the application of detailed balance, an expression was derived giving the forbidden to intercombination line ratio as a function of electron density. As to be expected, the ratio ranges from a maximum value (low density or zero density limit), and approaches zero for very large electron densities.

The Gabriel and Jordan electron density theory and their observations of inner-shell transitions in lithium-like ions precipitated a search for satellite lines in flare spectra and investigations of the resonance, intercombination, and forbidden lines of helium- 
like ions (Walker and Rugge, 1970; Doschek and Meekins, 1970; Neupert, 1971a; Doschek et al., 1971a, b; Walker and Rugge, 1971). The results of the search for satellite lines are reflected by Table I. Determinations of electron densities from observations of active regions and flares are summarized in Freeman et al. (1971). The main conclusions regarding electron densities are:

(1) Measurements of helium-like lines of the comparatively heavier elements (silicon, sulfur) give higher electron densities $\left(\sim 10^{14} \mathrm{~cm}^{-3}\right)$ than are found for the lighter elements $\left(10^{11} \mathrm{~cm}^{-3}\right.$; neon, oxygen $)$. Since the heavier elements emit more efficiently at higher temperatures than is the case for the lighter elements (see Figure 17), these results indicate that high temperature regions are also at relatively high electron densities.

(2) The electron densities are quite large; about two orders of magnitude larger than densities determined by other methods. For example, an electron density of $10^{14} \mathrm{~cm}^{-3}$ is indicated for sulfur (Walker and Rugge, 1970; Freeman et al., 1971). In a recent review (Neupert, 1970) typical densities of $10^{13} \mathrm{~cm}^{-3}$ are cited for flare plasmas, as determined by the Gabriel and Jordan theory. Because of the surprisingly high values of electron densities determined from the helium-like lines, further experimental and theoretical investigations are necessary before these densities can be accepted at face value.

With the exception of iron, and presumably elements such as nickel and chromium, the satellite lines observed in flare spectra are weak compared to the resonance lines of heliumlike ions. Even in the heavier elements, only the lithiumlike satellites produce substantial emission. Most of the satellite lines in Table I were first reported by Neupert (1971a) and Walker and Rugge (1971). The calcium satellites have been extensively investigated by the NRL group. The ability to resolve these features from nearby, intense lines, e.g., the forbidden lines of the helium-like ions, depends primarily on instrumental characteristics. The weak satellites can only be observed in the most intense flares.

Figure 13 shows many of the satellite lines reported by Neupert (1971a) and Walker and Rugge (1971) (see Table I for transitions), and additional satellite lines observed in the NRL spectrum. Walker and Rugge suggested that satellites to the hydrogenlike ions may also be present in flare spectra, and identified satellite lines of $\mathrm{MgXII}$ in their data. NRL data in Figure 13 and Goddard data in Figures 20 and 21 show the first clear evidence of such features for silicon. In the NRL data the emission group on the long-wavelength side of the Lyman- $\alpha$ line of SixIV is present in two spectral scans of this event. The long-wavelength 'edge' of the emission agrees with the wavelength of the ${ }^{1} D$ helium-like satellite of hydrogenic silicon. Emission on the short-wavelength side of the 'edge' would correspond to emission from the ${ }^{1} S,{ }^{1} P$, and ${ }^{3} P$ configurations of the doubly excited helium-like ion (Walker and Rugge, 1971). Table I gives the transitions suggested. It is extremely difficult to observe these lines because of their low intensity; the spectrum in Figure 13 (and companion scan) of the November 16, 1970 event is the only spectrum obtained by the NRL group which shows unequivocally an emission feature on the long-wavelength side of the SixIV line. On two 
scans of the November 2 event, the ${ }^{1} D$ feature appears to be present, having about the same intensity as the second order calcium feature nearby. However, emission to the short-wavelength side of this satellite line $\left({ }^{1} S,{ }^{1} P\right.$, and ${ }^{3} P$ configurations $)$ is definitely absent. The NRL group has also possibly observed two of these transitions for magnesium (see Table I) in the November 2, 1969 flare.

High resolution observations of lithium-like satellite lines of the helium-like silicon ions have been made by Walker and Rugge (1971). An example of their data is shown in Figure 19. The Sixil $\left(1 s^{2} 2 s^{2} S-1 s 2 s 2 p^{2} P\right)$ transition is clearly resolved from the forbidden line of Si XIII. Present instruments do not have the ability to resolve the ${ }^{2} D$ satellite from the forbidden line, i.e., SixII $\left(1 s^{2} 2 p^{2} P-1 s 2 p^{2}{ }^{2} D\right)$, and this line is expected to be about the same intensity as the ${ }^{2} P$ line (Gabriel, 1971a).
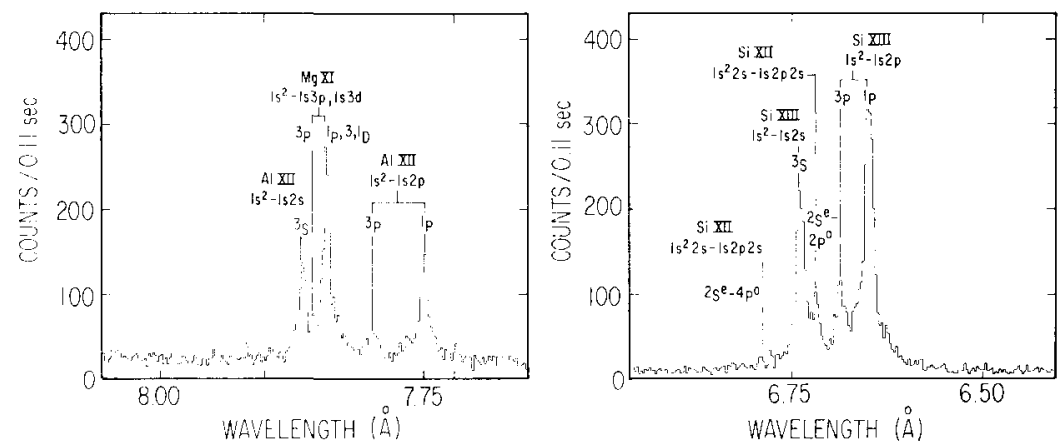

Fig. 19. High resolution EDDT spectra near $\sim 6.6 \AA$ and $7.8 \AA$. Shown are the heliumlike lines of AlXII and SiXIII. Other transitions identified as SixII are also apparent. Note, in particular, the line identified as SixII $\left(1 s^{2} 2 s^{2} S-1 s 2 s 2 p^{2} P\right)$. Spectra courtesy of A. B. C. Walker, Jr., Aerospace Corporation.

Figure 19 also shows an emission feature on the long-wavelength side of the forbidden line of Si XIII identified by Walker and Rugge as Si XII $\left(1 s^{2} 2 s^{2} S^{e}-1 s 2 s 2 p^{4} P^{0}\right)$. The same feature can be seen in the Goddard and NRL spectra of Figures 7, 9 and 13. Figure 13 shows these same transitions for calcium in the $\mathrm{LiF}$ spectrum.

Figure 19 also shows data near $8 \AA$ obtained by Walker and Rugge (1971). The helium-like aluminum lines are well-resolved, and Walker and Rugge identify emission at $7.776 \AA$ as an inner-shell transition in AlXI.

Other examples of suspected satellite lines in flare spectra are shown in Figures 20, 21, and 22. The data in Figures 20 and 21 were obtained by Neupert (1971a) and show possible satellite lines of hydrogenic ions and satellites due to lithium-like transitions near the helium-like lines. The NRL data of Figure 22 show the CaxvIII $\left(1 s^{2} 2 s^{2} S\right.$ $1 s 2 s 2 p^{2} P$ ) satellite well-resolved from the CaxIX forbidden line.

From observations of neon, silicon, and sulfur, Walker and Rugge (1971) conclude that the ratio of the $1 s^{2} 2 s^{2} S-1 s 2 s 2 p^{2} P$ satellite line to the resonance line of the heliumlike ion is 0.07 for neon, 0.16 for silicon, and 0.21 for sulfur. Neupert (1971a) concludes from similar observations that the ratio of combined emission from lithium- 
like and beryllium-like ions to the combined helium-like emission is 0.11 for silicon, 0.20 for sulfur, 0.14 for argon, and 0.76 for iron. For these same elements, Neupert finds that boron-like emission is only 0.02 of the helium-like emission. From observations of the helium-like calcium group near $3.2 \AA$, Doschek et al. (1971b) conclude

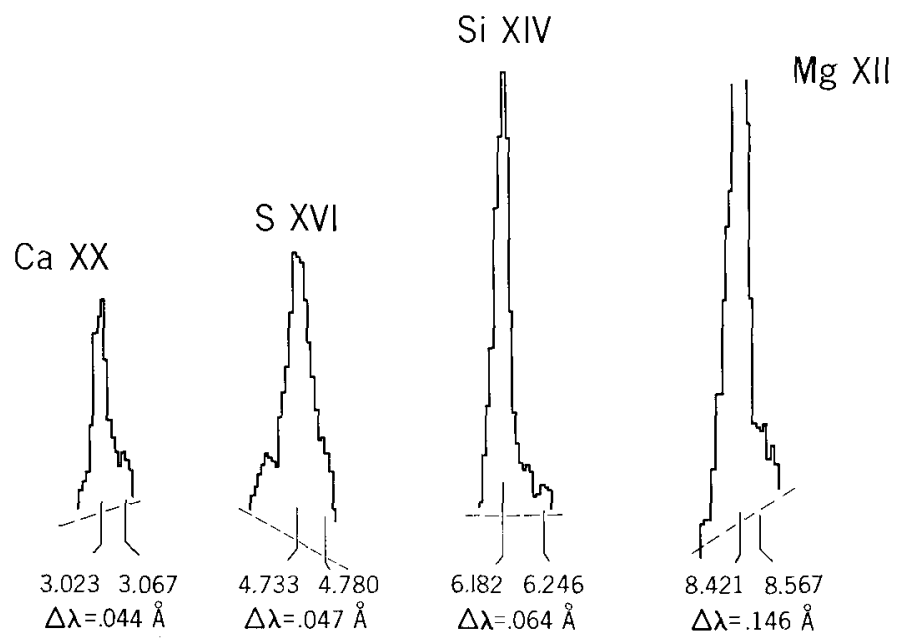

Fig. 20. Possible helium-like satellites seen on the long-wavelength side of the Lyman- $\alpha$ lines of hydrogen-like ions. Spectra courtesy of W. M. Neupert, Goddard Space Flight Center.

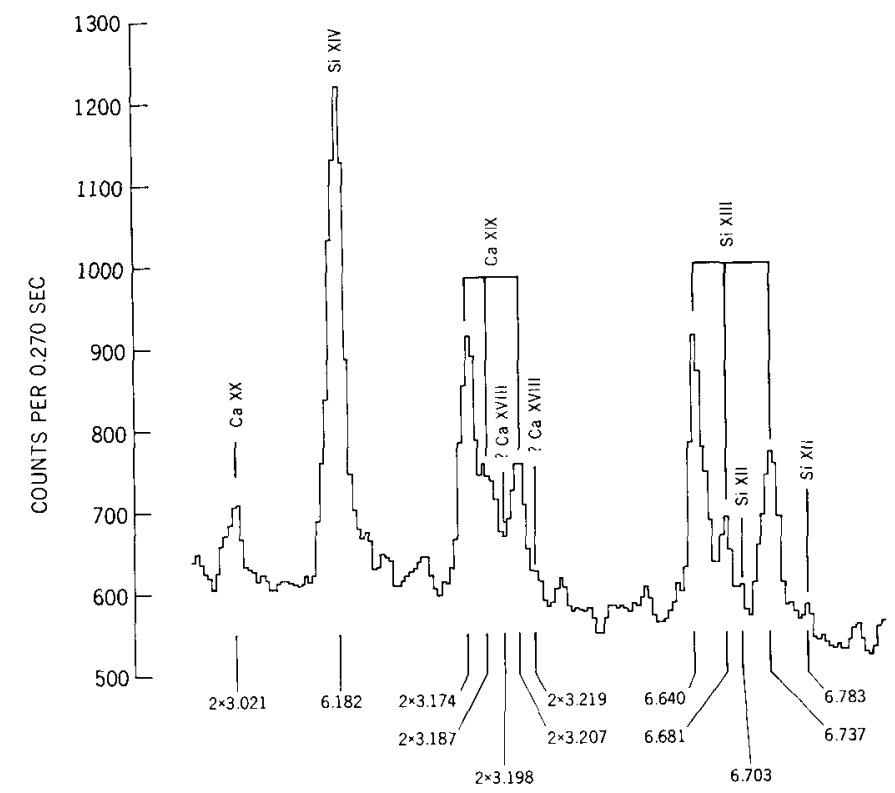

Fig. 21. Spectrum showing possible satellite lines to SixIV and Si XIII. Also present is a strong second order spectrum of CaxIx. By comparing the second order wavelengths of the calcium lines to the first order Lyman- $\alpha$ Sixıv line, accurate first order wavelengths of the Ca xIX lines can be derived (Neupert, 1971a). Spectrum courtesy of W. M. Neupert, Goddard Space Flight Center. 


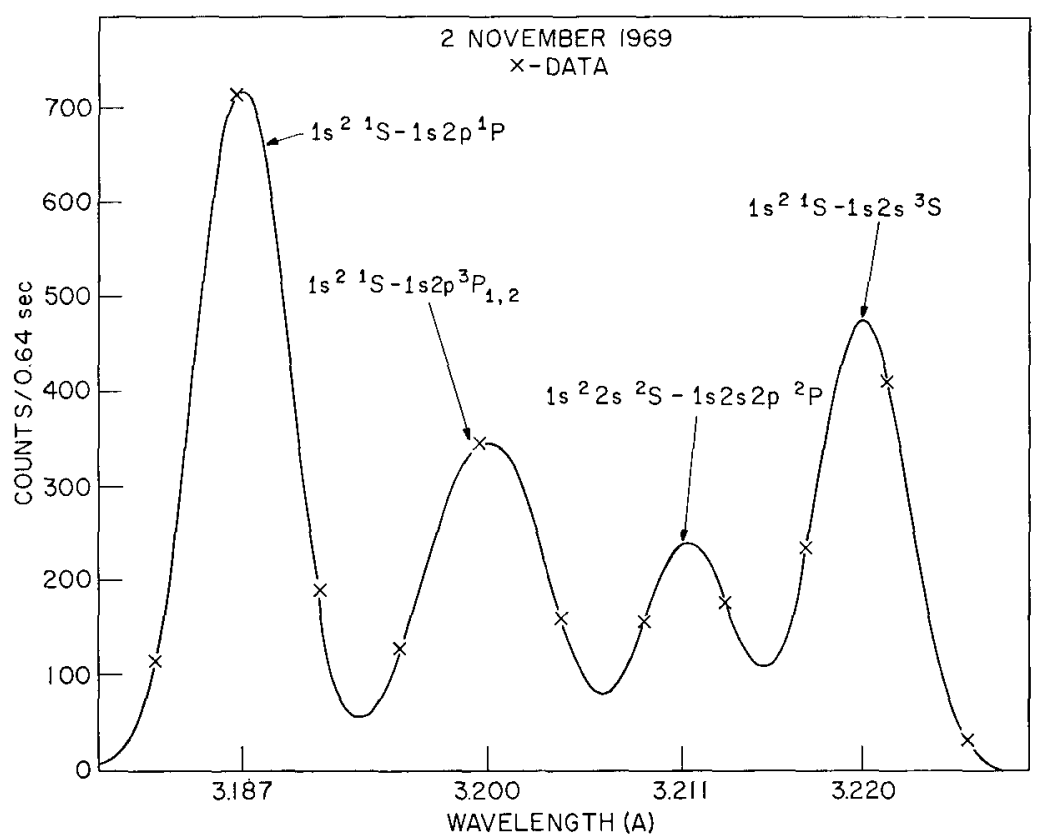

Fig. 22. NRL OSO-6 spectrum of the resonance, intercombination, and forbidden lines of CaxIX. Also shown is the CaxvIII $\left(1 s^{2} 2 s^{2} S-1 s 2 s 2 p^{2} P\right)$ satellite line. The data were fitted by a least squares technique described in Doschek et al. (1971b) that considers the instrumental profile and source size. The intercombination line is broader than the other lines due to $J$-splitting of the $2^{3} P$ level. Thus, the $2^{3} P_{2}-1^{1} S_{0}$ and $2^{3} P_{1}-1^{1} S_{0}$ lines are observed, but the $2^{3} P_{0}-1{ }^{1} S_{0}$ transition is strictly forbidden.

that the intensity ratio of the ${ }^{2} P$ lithium-like satellite to the resonance line of CaxIX is $\sim 0.3$. The intensity ratio of the combined emission due to lithium-like and beryllium-like ions of calcium relative to the combined emission due to the helium-like ion is $\sim 0.2$. For iron, Doschek et al. (1971a) find that the percentage emission due to inner-shell transitions appears to vary during flares. Typically, however, the intensity ratio of the ${ }^{2} P$ satellite to the resonance line of Fexxv is $\sim 0.6$.

It should be mentioned that, due to blending with the forbidden lines of the heliumlike ions, the $1 s^{2} 2 p^{2} P-1 s 2 p^{2}{ }^{2} D$ transition was neglected in deriving the above values. As previously mentioned, this transition should be about equal in strength to the ${ }^{2} P$ transition. Also, removing the ${ }^{2} D$ line will alter the electron density determinations; the effect being to raise the densities determined by the Gabriel and Jordan theory.

The conclusion obtained from observations of lithium-like satellite lines in active regions and flares is that the intensity of the satellites relative to the intensity of the helium-like lines increases with increasing atomic number. This observation is consistent with the hypothesis that the satellites, as in the laboratory, are formed by dielectronic recombination to the helium-like ion. Moreover, the relatively high intensity of the iron satellites may indicate that in the case of the heavier elements such as iron, and perhaps to a lesser extent for calcium and argon, collisional inner-shell excitation and/or ionization may also contribute substantially to the line strengths. 
Although it appears that helium-like satellite lines are present in flare spectra, the low intensity of the observed features and small uncertainties in the wavelengths preclude positive identifications in most cases until higher resolution observations with more sensitive instruments are obtained. Walker and Rugge (1971), however, regard their identifications of MgXI satellite lines as quite certain. They also calculate the intensity ratio of the ${ }^{1} \mathrm{D} \mathrm{MgXI}$ satellite line to the Lyman- $\alpha$ line of $\mathrm{MgXI}$ under the assumption that the ${ }^{1} D$ level is formed by dielectronic recombination to $\mathrm{MgXII}$. The theoretical result compares well with their experimental intensity ratio.

The following picture is indicated regarding the formation of innershell transitions in flare plasmas. For the light ions, such as magnesium and silicon, the electron temperatures are too high for appreciable abundances of lithiumlike and lower ionization stages to exist. Therefore, collisional excitation of the lithium-like ion, for example, cannot compete with dielectronic recombination to the much more abundant heliumlike ion. Also, because autoionization probabilities are larger relative to stabilizing probabilities in the lighter ions than for the heavier ions, the satellite lines will be weak compared to the resonance lines of helium-like ions. For heavier elements such as iron, however, an appreciable abundance of FexxIv, FexxiII, and FexxII can exist at electron temperatures $\$ 20 \times 10^{6} \mathrm{~K}$. This factor, combined with the reduced autoionization probabilities compared to stabilizing rates, may combine to produce the relative increases in satellite line strengths with increasing atomic number, and may explain the relatively intense satellites of iron.

Furthermore, observations obtained to date do not appear to support a non-thermal interpretation (Blake and House, 1971) in which hot electrons impinge on a much cooler gas $\left(\sim 10 \times 10^{6} \mathrm{~K}\right)$ producing inner-shell excitations in an ion distribution characteristic of the low temperature plasma. It appears likely that such a hot-electron component would heat the low temperature gas within seconds (Spitzer, 1967) by elastic electron-electron Coulomb collisions and destroy the low-temperature ion distribution. Such effects are not observable with the time-resolution of existing instruments. In this regard, however, there have been some observations of satellite line enhancements during flares that may suggest a heating of cooler, surrounding plasma (Doschek $e t$ al., 1971b). These observations will be discussed in Section 5.

\section{THE SOFT X-RAY CONTINUUM}

The soft X-ray continuum observed during solar flares is assumed to be produced primarily by free-free and free-bound processes in an optically thin thermal plasma. Based on this assumption, electron temperatures $\sim 15-40 \times 10^{6} \mathrm{~K}$ have been estimated (Culhane and Phillips, 1970b; Meekins et al., 1970; Neupert, 1970). However, it has been suggested that the continuum emission $<3 \AA$ and $\gtrsim 1 \AA$ might contain a nonthermal contribution due to either monoenergetic electrons or electrons distributed in energy according to a power law (Kahler and Kreplin, 1971; Elwert and Haug, 1970). Recent observations made by Tindo et al. $(1970,1971)$ show a marked polarization around $12 \mathrm{keV}$, i.e., $\sim 1 \AA$, during the initial 'flash' phase of intense flares, which is interpreted by them as emission from non-thermal electrons. If the hard $\mathrm{X}$ rays are 
indeed produced by a non-thermal electron distribution, there is no contradictory evidence that these high energy electrons do not have a low energy cutoff extending beyond $1 \AA$, and hard X-ray data obtained to date do not rule out this possibility (Kane, 1969; Kahler and Kreplin, 1971). However, the hard X-ray data indicate that such non-thermal emission should be observable only during the initial heating phase of flares and should last for only a few minutes.

At present, therefore, it would appear that inspection of soft X-ray spectrometer data offers the best possibility of determining the source of the continuum. However, while it is true that the soft X-ray continuum has a spectral shape that is generally consistent with thermal emission (Culhane et al., 1969), a detailed comparison with theoretical thermal spectra is not presently feasible because:

(1) the source region is multithermal (Meekins et al., 1970; Beigman et al., 1971b; Neupert, 1970), which significantly complicates comparisons of observed and theoretical spectra,

(2) the continuum recorded by crystal spectrometers is distorted by multiple diffraction and poor time resolution,

(3) proportional counters have poor spectral resolution,

(4) uncertainties in theoretical cross-sections and relative abundances of the elements introduce uncertainties in the theoretical spectrum, and,

(5) weak emission lines, and the two-photon continuum produced by the decay of the $2{ }^{1} S$ states of heliumlike ions and the $2{ }^{2} S$ states of hydrogenic ions complicate the observed spectral distribution.

Therefore, detection of a non-thermal component in the continuum above $1 \AA$ requires: (1) accurately calibrated crystal spectrometer measurements with high time and spectral resolution at selected wavelengths, (2) polarization measurements in the $3 \AA$ range over the duration of an event, and (3) improvements in theoretical crosssections and determinations of solar element abundances.

However, excluding the initial heating phase it appears likely that most of the observed continuum is indeed thermal (Culhane, 1969). Electron temperatures determined from line to continuum ratios are consistent with temperatures determined from line to line ratios (Meekins et al., 1970; Neupert, 1970).

Searches for radiative recombination edges in the continuum have been unsuccesful. The SXvi edge reported by Meekins and Doschek (1970) is probably an effect produced by the very weak (in the spectrum examined) K xviII lines. Inspection of Figure 13, the most intense flare observed by the NRL group, does not show an obvious indication of the Sixv recombination edge at $4.65 \AA$, an edge that might be expected at the high electron temperatures $\left(\sim 25 \times 10^{6} \mathrm{~K}\right)$ determined for this event. The position of the edge is marked in the figure. Observation of this flare and observation of another intense flare on August 12,1970 during the decay phases of these events did not reveal any indication of the Sixv edge. Similarly, edges due to recombinations of other ions of silicon and other elements have not been detected.

It is also difficult to detect continuum emission from the two-photon decay of the $2{ }^{1} S$ and $2{ }^{2} S$ states of heliumlike and hydrogenic ions, respectively. The low intensity, 
coupled with the broad emission maximum expected, and the weak lines and rapidly changing flux encountered in flare spectra, have so far precluded detection of these features.

Meekins and Doschek (1970) have discussed continuum edges in the NRL spectra which apparently are not due to instrumental effects and cannot be produced by radiative recombination. One of these edges can be seen $\sim 2.8 \AA$ in the EDDT spectrum of Figure 9. An explanation was attempted in terms of radiative-Auger processes. However, it now appears that very weak emission lines may produce the effect of an edge over the wavelength ranges involved.

\section{ELEMENT ABUNDANCES FROM FLARE SPECTRA}

As of yet, there has been no quantitative attempt to determine element abundances from flare spectra. However, a qualitative estimate of relative abundances of certain elements can be made by inspecting Figures 7, 9, and 13. From the spectra in these figures, it is clear that the abundances of manganese is less than the abundances of nickel and chromium, and the abundance of nickel may be somewhat greater than the abundance of chromium, i.e., the excitation energy of nickel is greater than the excitation energy of chromium. Similarly, the abundance of titanium is less than the abundance of chromium and the abundances of all four of these elements are considerably less than the abundance of iron. Even with well-determined instrumental efficiencies and temporal effects in the data removed, the low intensity of the $\mathrm{Ni}, \mathrm{Mn}, \mathrm{Cr}$, and $\mathrm{Ti}$ lines would make more quantitative estimates difficult.

At longer wavelengths, it is apparent from the above figures that the abundance of potassium is much less than the abundance of calcium. The relative abundances of iron and calcium are difficult to infer from the spectra because of instrumental efficiencies and the greater excitation energy of iron.

It also appears that the abundance of argon is somewhat less than the abundance of calcium, i.e., compare ArXVII and CaXIX in Figure 7, and the Lyman- $\beta$ lines of ArXVII and CaXIX in Figure 9. Finally, from a comparison of the Lyman- $\beta$ lines of silicon and aluminum in Figure 13, it is clear that the aluminum abundance must be less than the silicon abundance.

From observations of the Fexxiv $\left(1 s^{2} 2 s^{2} S-1 s^{2} 4 p^{2} P\right)$ lines at $\sim 8.0 \AA$ and the Lyman- $\alpha$ MgXII line at $8.421 \AA$, it should be possible to determine the relative abundances of iron and magnesium if the temperatures and emission measures of the plasmas producing the FexXIV and MgXII emission were known. Unfortunately, the temperature and emission measure structures of flare plasmas have not been determined and assumptions must therefore be made in order to determine the relative abundance of iron to magnesium in this manner. If, for the NRL data of the November 2, 1969 event, it is assumed that FexXIV emission is produced at $25 \times 10^{6} \mathrm{~K}$, and that the MgXII line is formed in (a) an $\sim 10 \times 10^{6} \mathrm{~K}$ plasma, or (b) an $\sim 25 \times 10^{6} \mathrm{~K}$ plasma, then assuming equal emission measures for iron and magnesium in both cases, and that the lines are formed by collisional excitation, the iron to magnesium abundance is (a) 0.74 , and, (b) 0.13 . Both of these values are consistent with recent relative abun- 
dance determinations of iron and magnesium because of the large uncertainty in the iron abundance (Hauge and Engvold, 1970). Nevertheless, because of the assumptions necessary to calculate the abundance ratio from the flare data, these calculations should at present be regarded as nothing more than an interesting exercise.

\section{Time-Behavior of Spectral Emission Features During Flares}

\section{A. IONIZATION EQUILIBRIUM AND LINE FORMATION}

Soft X-ray bursts differ markedly in overall timescales. As mentioned in connection with the broadband results (Section 3A), some bursts may evolve in a minute or less while others are detectable for hours after the initial enhancement of X-ray flux. To obtain a reasonable estimate of emission line behavior throughout an event from existing crystal spectrometer experiments, it is necessary for significant variations in line intensity to occur over time intervals of $\sim$ five minutes. For time intervals shorter than this, important modulations in intensity might not be detected, while for longer time intervals, the spacecraft may enter darkness before appreciable variations in flux occur. Most of the instruments flown to date have not had the capability of observing intensity fluctations $<1 \mathrm{~min}$. The exception is the instrument flown by Neupert et al. (1969) on OSO 3 which had the capability to monitor a line continuously with a time resolution of $\sim 30 \mathrm{~s}$. Figure 23 shows the iron-line blend at $1.9 \AA$ monitored during the rise and decay of a large flare. Also shown is the time-history of a line at $11.8 \AA$, which Neupert suggests may be due to FexxII. (Conclusive evidence exists

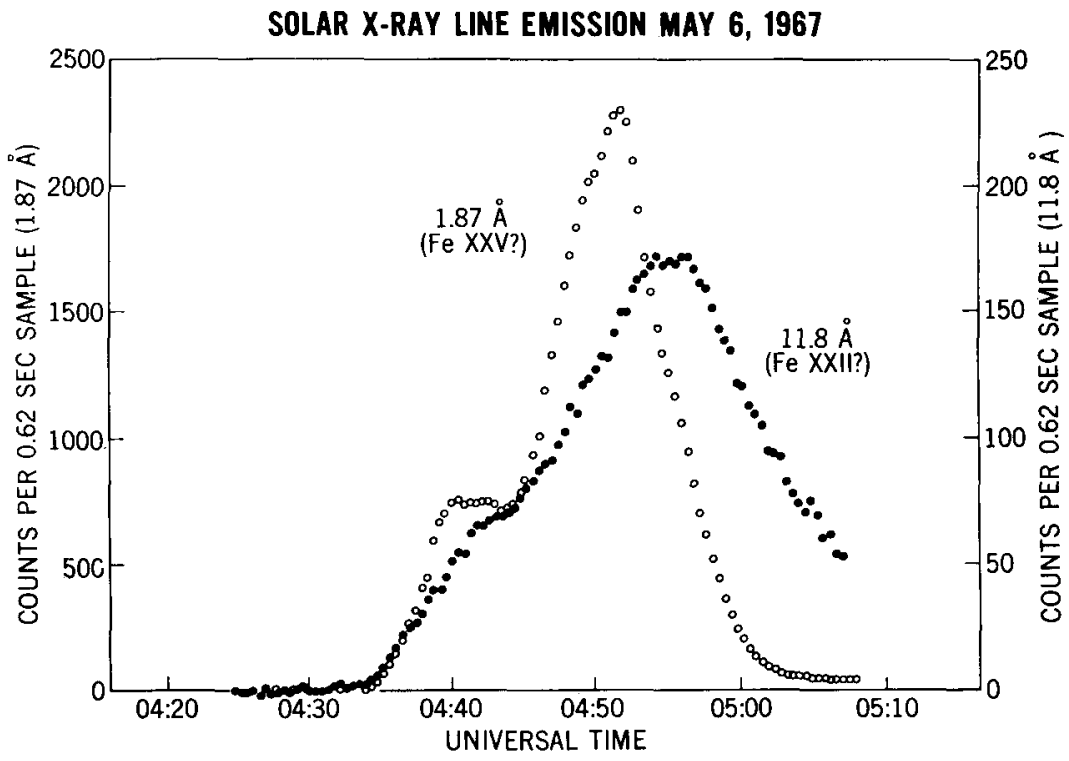

Fig. 23. Time-history of emission lines at $1.87 \AA$ and emission at $11.8 \AA$. Emission at $1.87 \AA$ contains substantial contributions due to Fexxiv as well as Fexxv. Note the marked inflection in the $1.87 \AA$ profile. An indication of this inflection is seen in the $11.8 \AA$ line. Data courtesy of W. M. Neupert, Goddard Space Flight Center. 
that this line is due to a high iron ionization stage (Neupert, 1971b). However, the line could possibly be due to FexxIII, or FexXI, although Neupert considers this unlikely.) There are two interesting features of these profiles. First, there is an inflection in the rising portion of the $1.9 \AA$ feature, which demonstrates that the heating of the soft $\mathrm{X}$-ray emitting plasma is not necessarily uniform, and secondly, the peak flux in the longer wavelength line occurs later than the peak flux in the $1.9 \AA$ blend, and the decay of the longer wavelength line is slower than the decay of the $1.9 \AA$ blend. The NRL group has observed both kinds of behavior in the large flare of November 2, 1969, i.e., irregular increases in line strength and earlier peaking of the shorter wavelength lines. However, the variations described were observed for lines other than those above, and the actual changes in line intensity were quite different from the Goddard results. Apparently, flares can differ markedly in detail; the November 2 results will be further discussed in Section 5B.

An example of the differing time-scales encountered in soft X-ray events is shown in Figure 24. The Lyman- $\alpha$ SixIv lines for these events were monitored discontinuously by the NRL OSO-6 spectrometers. The dashed lines indicate possible intensity behavior between the data points. Also shown in Figure 24 are the uncorrected counting rates in the continuum underlying the silicon lines. Figure 24 shows that the November 15, 1970 event was more impulsive than the November 2, 1969 event ; consequent$1 \mathrm{y}$, the indicated behavior between the data points is more uncertain for the November 15 flare than for the November 2 event. It is interesting to compare these two events with the OSO-3 flare of Figure 23.

Figures 23 and 24 suggest that the assumption of ionization equilibrium in flares should be carefully examined. If the flare plasma constitutes a current such that mass motion is significant over the time-scales involved in ionization and recombination, then a divergence term must be added to the ionization equilibrium equations (Neupert, 1964). Also, if the heating and cooling time-scales are comparable to the timescales involved in achieving ionization equilibrium, then time-dependent ionization equilibrium equations should be used (Burton and Wilson, 1961). Present instrumentation is only marginally capable of detecting mass motions in soft X-ray flares; however, the effect of such motions may be approximated by using time-dependent equations. For each ion $N_{z}$,

$$
\frac{\mathrm{d} N_{z}}{\mathrm{~d} t}=N_{\mathrm{e}}\left(N_{z-1} Q_{z-1}+N_{z+1} \alpha_{z+1}\right)-N_{\mathrm{e}} N_{z}\left(Q_{z}+\alpha_{z}\right) .
$$

Equations (4) state that the net rate of change of the number density of an ion with net charge $z$ is equal to the gain per unit time by ionization of ion $N_{z-1}$ and total recombination to ion $N_{z+1}$, minus the loss per unit time by ionization and total recombination of ion $N_{z}$.

To clarify the time-scales involved in reaching ionization equilibrium under conditions in flare plasmas, Equations (4) were solved for silicon and sulfur under the following conditions. It is assumed that all of the silicon and sulfur is in the helium-like ionization stage at time $t=0$. This is a resonable approximation for conditions in a 


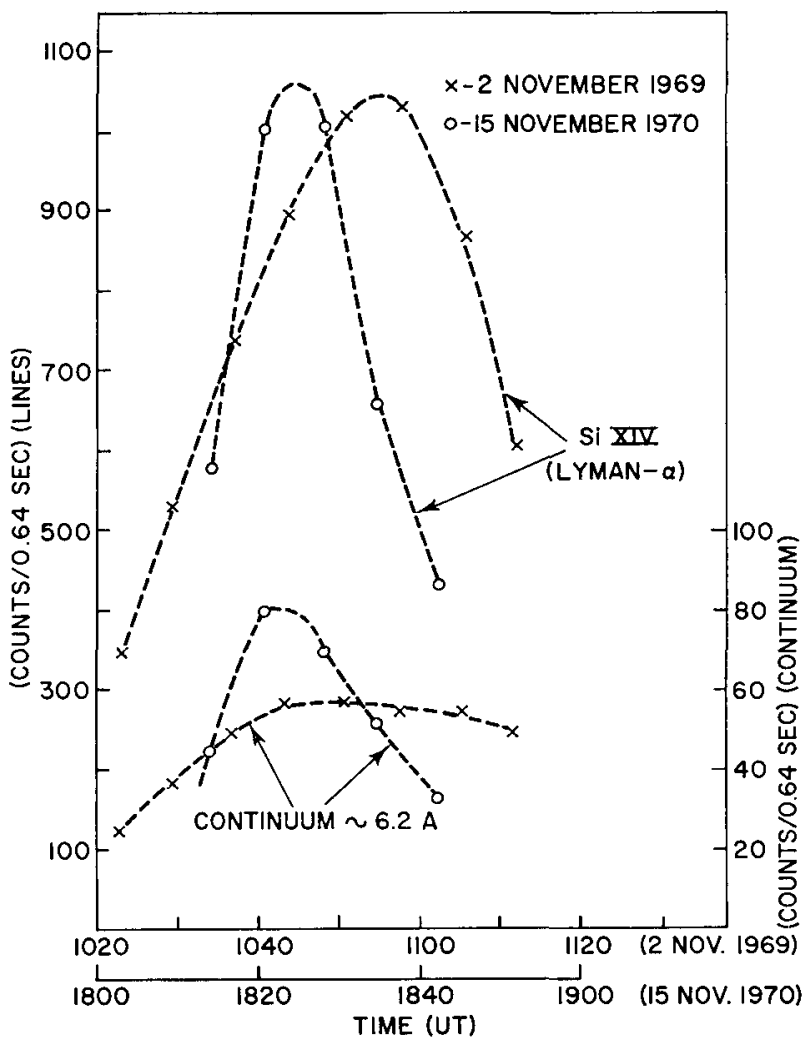

Fig. 24. NRL time-histories of the Lyman- $\alpha$ SixIV lines and continuum emission for two large events (OSO 6). Note the more impulsive nature of the November 15 event. Compare with Figure 23.

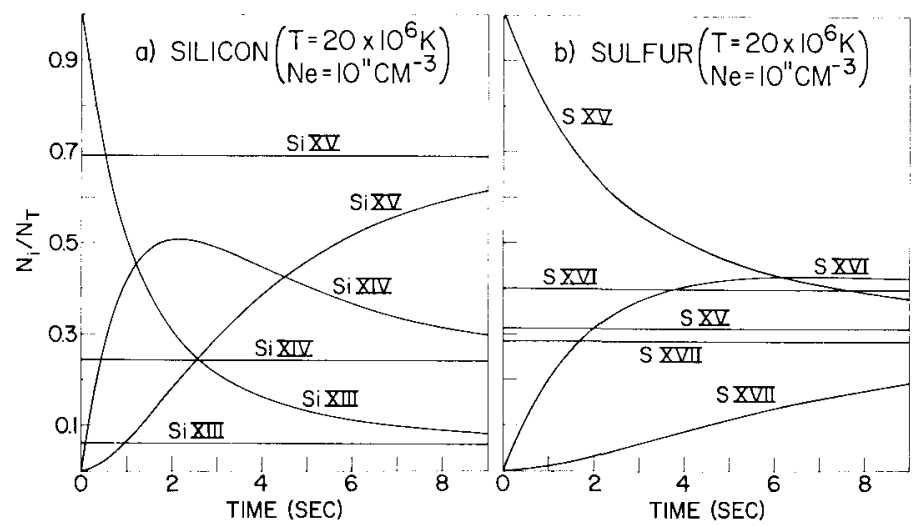

Fig. 25. Theoretical time-dependence of ionization of silicon and sulfur as described in text. Horizontal lines are equilibrium values of fractional abundances at $20 \times 10^{6} \mathrm{~K} . N_{\mathrm{i}}$ is the ion number density at time $t, N_{t}$ is the element number density. 
precursor, or a hot active region, i.e., electron temperatures $\sim 10 \times 10^{6} \mathrm{~K}$ (see Section $3 \mathrm{~A}$ ). It is also assumed that the plasma is instantaneously heated to $20 \times 10^{6} \mathrm{~K}$ at an electron density of $10^{11} \mathrm{~cm}^{-3}$. The higher densities based on forbidden to intercombination line ratios will shorten the time-scales proportionately.

The solutions of Equations (4) are shown in Figure 25. Plotted are the fractions of the total element abundances in the helium-like, hydrogen-like, and completely ionized ionization stages. For temperatures $\sim 20 \times 10^{6} \mathrm{~K}$ the fraction of sulfur and silicon in lower ionization stages than heliumlike may be neglected for the purposes of this discussion. The horizontal lines represent the equilibrium fractions at $20 \times 10^{6} \mathrm{~K}$. The cross-sections used in the calculations were the same as those used to obtain the results shown in Figure 17.

From Figure 25, equilibrium is almost achieved in a time of $20 \mathrm{~s}$. Similar timescales would apply to a gas that is cooling from $20 \times 10^{6} \mathrm{~K}$ to a lower temperature. For $N_{\mathrm{e}}=10^{12} \mathrm{~cm}^{-3}$, equilibrium would be reached in about one second, while for lower densities, equilibrium would be achieved on time-scales of the order of minutes. The temporary rise above the equilibrium value of the emission from the hydrogen-like ions of silicon and sulfur in Figure 25 is due to the dominance of ionization of the heliumlike ions over the ionization of the hydrogenlike ions during the initial phase of the heating.

From the short times needed to reach ionization equilibrium for $N_{\mathrm{c}} \gtrsim 10^{11} \mathrm{~cm}^{-3}$, it follows that a continuous injection of ions, or a current must exist in the flare plasma in order for a non-thermal ion distribution to be maintained and observed over the typical time-scales of Figures 23 and 24. Furthermore, confinement of the plasma must be minimal; or the emission measure of the thermalized ions would dominate the non-thermal contribution. Conversely, if the flare plasma is concentrated and confined in magnetic ribbons and loops as is indicated by X-ray photographs, then the X-ray spectrum of the plasma can possibly be interpreted as thermal emission from a multitemperature source. On the other hand, for $N_{\mathrm{e}}$ appreciably less than $10^{11} \mathrm{~cm}^{-3}$, some departure from ionization equilibrium may be observable.

Another theoretical problem in interpreting line emission from flares is the mechanism of line formation. Three processes can contribute to the line intensities: (1) collisional excitation, (2) dielectronic recombination, and (3) radiative recombination. Cross-sections for collisional excitation of optically allowed lines have been derived from theory and laboratory data by Van Regemorter (1962). Radiative recombination coefficients $\alpha_{n}^{l}$ into the $n, l$ levels of hydrogenic ions have been tabulated by Burgess (1964b). A comparison of these rates for the Lyman- $\alpha$ line of SixIV at $20 \times 10^{6} \mathrm{~K}$, for example, shows that the collisional excitation coefficient $(C)$ is greater by a factor of $\sim 50$ than the radiative recombination coefficient, i.e., $\mathrm{C}\left(1^{2} S-2^{2} P\right)=5.2 \times 10^{-12} \mathrm{~cm}^{3}$ $\mathrm{s}^{-1}, \alpha_{2,1}=\sim 1.0 \times 10^{-13} \mathrm{~cm}^{3} \mathrm{~s}^{-1}$. Cascading from higher levels will not effect the result significantly. Under conditions of equilibrium, therefore, collisional excitation should dominate radiative recombination. This condition would be upset if line emission in flares is produced by highly ionized elements recombining in a very low temperature plasma $\left(T \approx 5 \times 10^{6} \mathrm{~K}\right)$. Under these conditions radiative recombination could 
be the dominant mechanism of line production. It should be possible with existing data to determine the importance of radiative recombination. For a pure recombination spectrum of silicon produced in a $20 \times 10^{6} \mathrm{~K}$ plasma, the ratio of Lyman- $\alpha$ SiXIV to Lyman- $\beta$ SixIV is $\sim 4$. If collisional excitation and dielectronic recombination are dominant, however, then the ratio would be about twice as large.

The contribution of dielectronic recombination to the resonance line intensities is more difficult to estimate. Blake (1968b) and Walker and Rugge (1971) have discussed the process

$$
1 s+\mathrm{e} \rightarrow 2 p n l \rightarrow 1 s n l+h v, \quad n, l \gg 1,
$$

which theory indicates might produce radiation at resonance line wavelengths in hydrogenic ions (Burgess, 1965). Walker and Rugge (1971) suggest that the contribution of dielectronic recombination to line fluxes in flares may be significant for the heavier ions such as iron. Other dielectronic recombination processes that may lead to line radiation at wavelengths close to the forbidden lines of heliumlike ions have been suggested by Doschek and Meekins (1970).

The importance of dielectronic recombination in flare plasmas needs further clarification. For example, if the dielectronic recombination rates into specific autoionizing levels of lithium-like ions can be accurately calculated, then, from observations of resonance lines of helium-like ions and the associated lithium-like satellites, it will be possible to calculate the electron temperature of the plasma producing the helium-like line emission (Gabriel, 1970).

\section{B. OPTICALLY ALLOWED LINES}

Resonance line behavior during soft X-ray bursts has already been illustrated in Figures 23 and 24. Resonance line behavior during the November 2, 1969 event, deduced from NRL OSO-6 spectra, is discussed below.

Eight spectral scans were obtained of the limb flare of November 2, 1969 during the time of peak X-ray flux in the hydrogen-like and helium-like emission lines. An analysis of the lines of hydrogen-like and helium-like magnesium ions has been given by Kreplin (1970), and the helium-like calcium lines and the iron-line blend near $1.9 \AA$ have been considered by Doschek et al. $(1971 \mathrm{a}, \mathrm{b})$. The data show that the peak intensities in the iron, calcium, and magnesium lines were not coincident; the iron-line blend peaked $\sim 1040 \mathrm{UT}$, the calcium lines peaked a few minutes later, and the hydrogen-like magnesium line peaked $\sim 1100$ UT. The obvious explanation of these effects is that the flare plasma is cooling, therefore progressively favoring emission from ions that exist in equilibrium at lower temperatures.

A sample of the silicon and sulfur data for this event is shown in Figure 26. The data are uncorrected counting rates taken from the NRL EDDT spectrometer, and the numbers over the emission features correspond to the numbered transitions given in Figure 26a. As the flare progresses, Figure 26 shows that the intensity of the SixIII $\left(1 s^{2}{ }^{1} S-1 s 3 p^{1} P\right)$ line increases relative to the intensity of the SixIV $\left(1 s^{2} S-3 p^{2} P\right)$ line, 


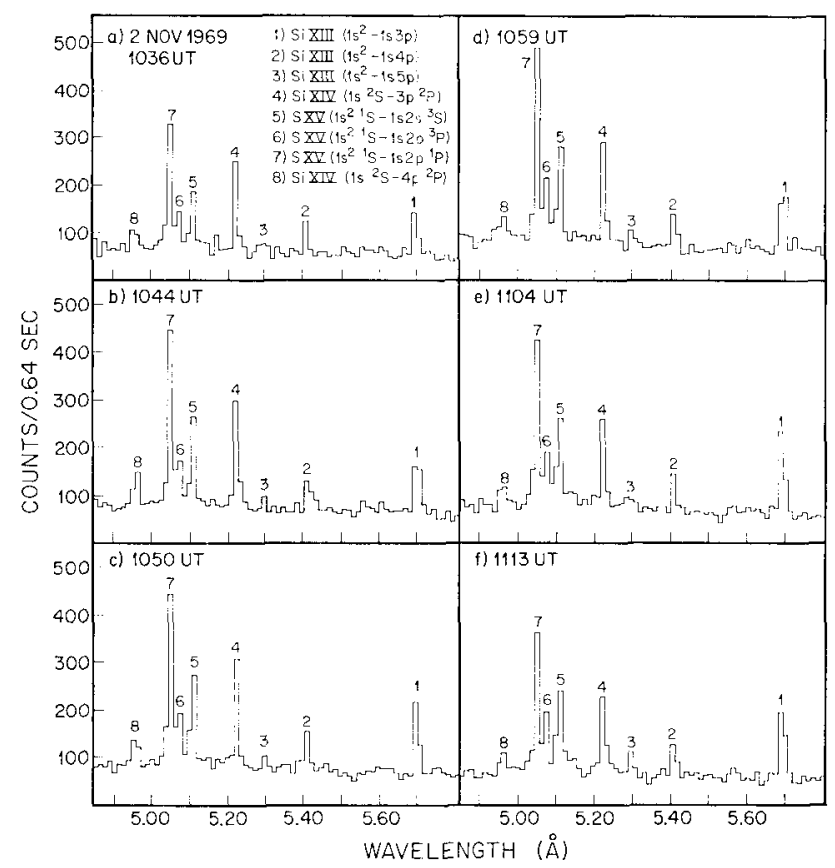

Fig. 26. Portions of the NRL EDDT crystal spectrometer spectra for six scans of the November 2, 1969 limb flare (OSO 6).

i.e., Lyman- $\beta$, and that the $\mathrm{Sxv}$ group gains in strength relative to the silicon lines. This observation is also consistent with a cooling plasma, regardless of the mechanism of line formation. In Figure 26f, the SixIII $\left(1 s^{21} S-1 s 5 p^{1} P\right)$ line is quite pronounced.

Unfortunately, instrumental correction parameters for the NRL OSO-6 spectrometers are still in preparation. Also, it is not valid to apply the NRL OSO-4 instrumental parameters (see Figure 4) to the OSO-6 instruments because the mica window in the OSO-6 detector is considerably thicker than the OSO-4 window. The lines in Figure 26, however, are sufficiently close in wavelength to compare relative intensities. The intensity ratio of the Lyman- $\beta$ SixIV line to the Lyman- $\gamma$ SixIV line is $\sim 2.8$, which is not in serious disagreement with either the ratio expected assuming collisional excitation and dielectronic recombination $\left(\sim 3.3\right.$ at an assumed temperature of $\left.20 \times 10^{6} \mathrm{~K}\right)$ as the dominant excitation process, or radiative recombination as the main process, which gives a ratio of $\sim 2.3$ at $20 \times 10^{6} \mathrm{~K}$. The low counting rates in these lines preclude precise determinations of line ratios.

Figure 27 shows the time histories of the Lyman- $\alpha$ lines of silicon and sulfur and the time histories of the resonance lines of the corresponding helium-like ions. Again, the conclusion that the flare is cooling, at least after $1050 \mathrm{UT}$, is clear from the behavior of the silicon lines. The fairly abrupt increase of a factor of $\sim 2$ in the intensity of the resonance line of SiXIII $\sim 1040$ UT appears to be real because time-history plots of the SixuI $\left(1 s^{2}{ }^{1} S-1 s 3 p{ }^{1} P\right)$ and SixIII $\left(1 s^{2}{ }^{1} S-1 s 4 p{ }^{1} P\right)$ transitions shown in Figure 26 ex- 

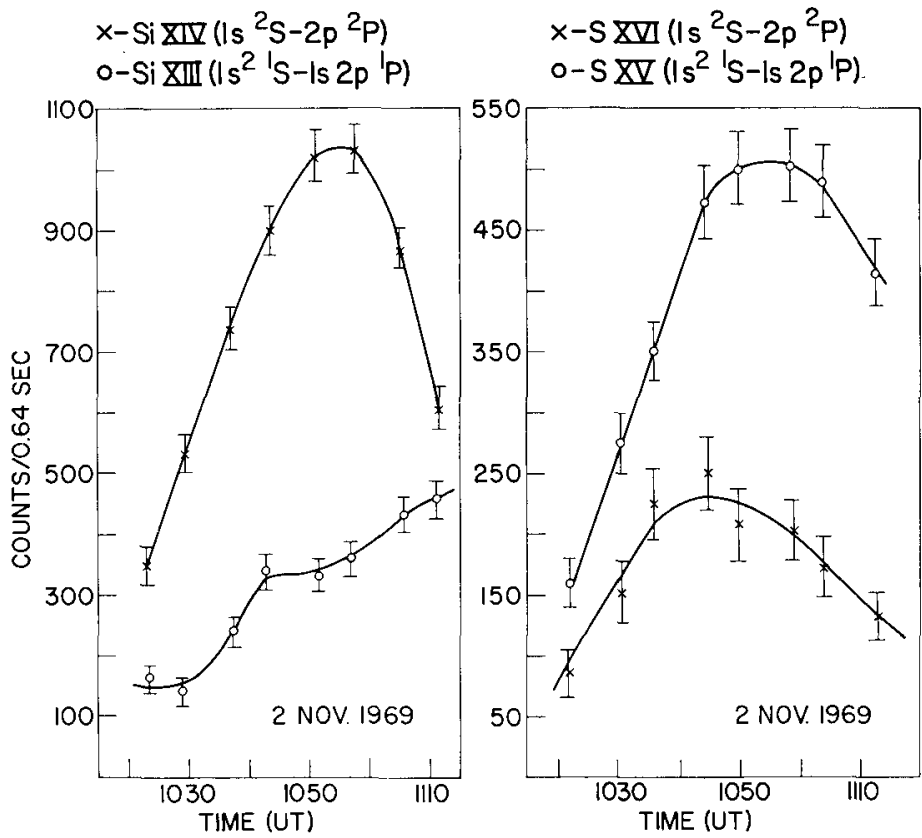

Fig. 27. Time-histories of the Lyman- $\alpha$ lines of silicon and sulfur and time-histories of the corresponding helium-like resonance lines from NRL spectra of the November 2, 1969 event (OSO 6).

hibit the same effect. Figure 27 also shows that the peak flux in the Lyman- $\alpha$ Si XIv line occurs about seven minutes later than the peak flux in the Lyman- $\alpha$ S XVI line, and that the helium-like sulfur line decreases in intensity after 1100 UT while the intensity of the resonance line of helium-like silicon continues to rise. The intensity ratios of the Lyman- $\alpha$ lines compared to the resonance lines of the helium-like ions of silicon and sulfur are shown in Figure 28. The ratios plotted have been determined by applying NRL OSO-4 instrumental correction parameters to the OSO-6 data, and therefore are uncertain. Nevertheless, it is clear from the figure that both the silicon and sulfur ratios decreased by about a factor of two in the time interval between $\sim 1030$ UT and 1110 UT. The decrease in the sulfur ratio appears fairly uniform, and the dip in the silicon ratio is due to the increase in the Si XIII lines discussed above. Figures 27 and 28 give strong support to the conclusion that the temperature of the flaring plasma decreased throughout the period of our observations. If the mechanism of line formation is assumed to be electron impact excitation, then the observations indicate a temperature change greater than $5 \times 10^{6} \mathrm{~K}$ between $\sim 1030 \mathrm{UT}$ and $1110 \mathrm{UT}$. Definite temperatures cannot be assigned until instrumental corrections are available. Finally, we note that the second order iron-line data of this event shown in Figure 29 also support the cooling hypothesis, because the line to continuum ratio of the resonance line of Fexxv is much larger near the beginning of our observation period (Figures 29a and $29 \mathrm{~b}$ ) than near the end of the observations (Figures $29 \mathrm{~g}$ and 29h). (We note that most of the continuum in Figure 29 is first order continuum, i.e., radiation $\sim 3.7 \AA$. Thus, 


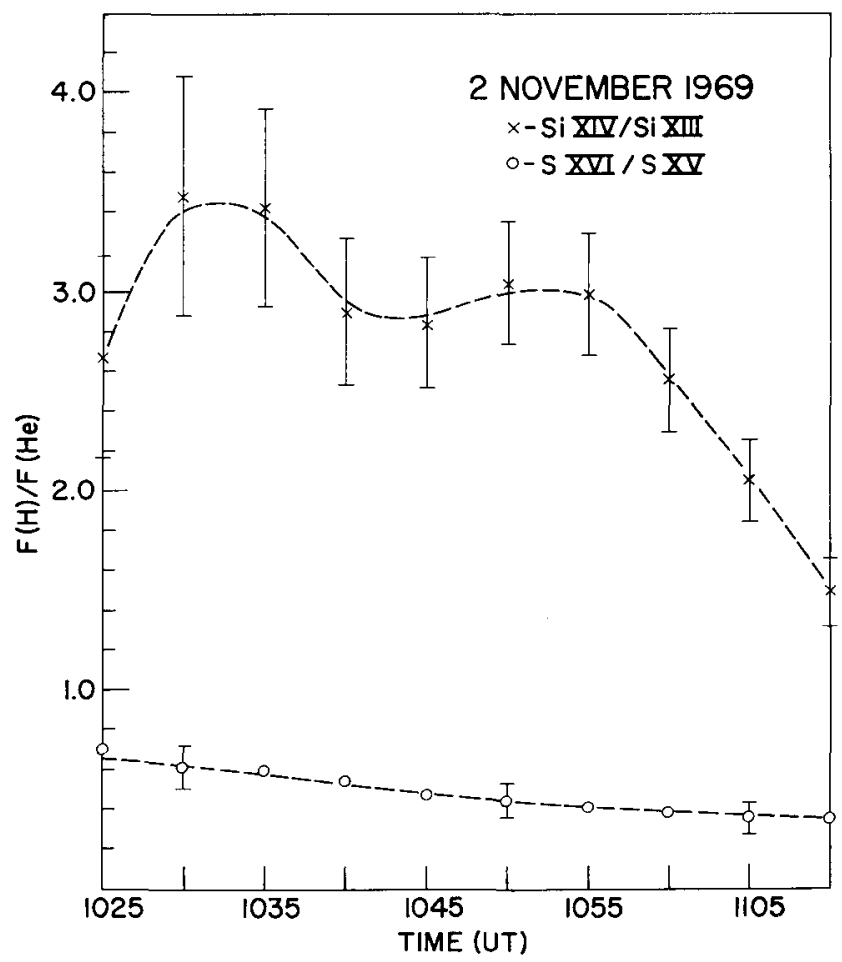

Fig. 28. Resonance line ratios of the hydrogen-like ion to the helium-like ion of silicon and sulfur from NRL spectra of the November 2, 1969 event (OSO 6).

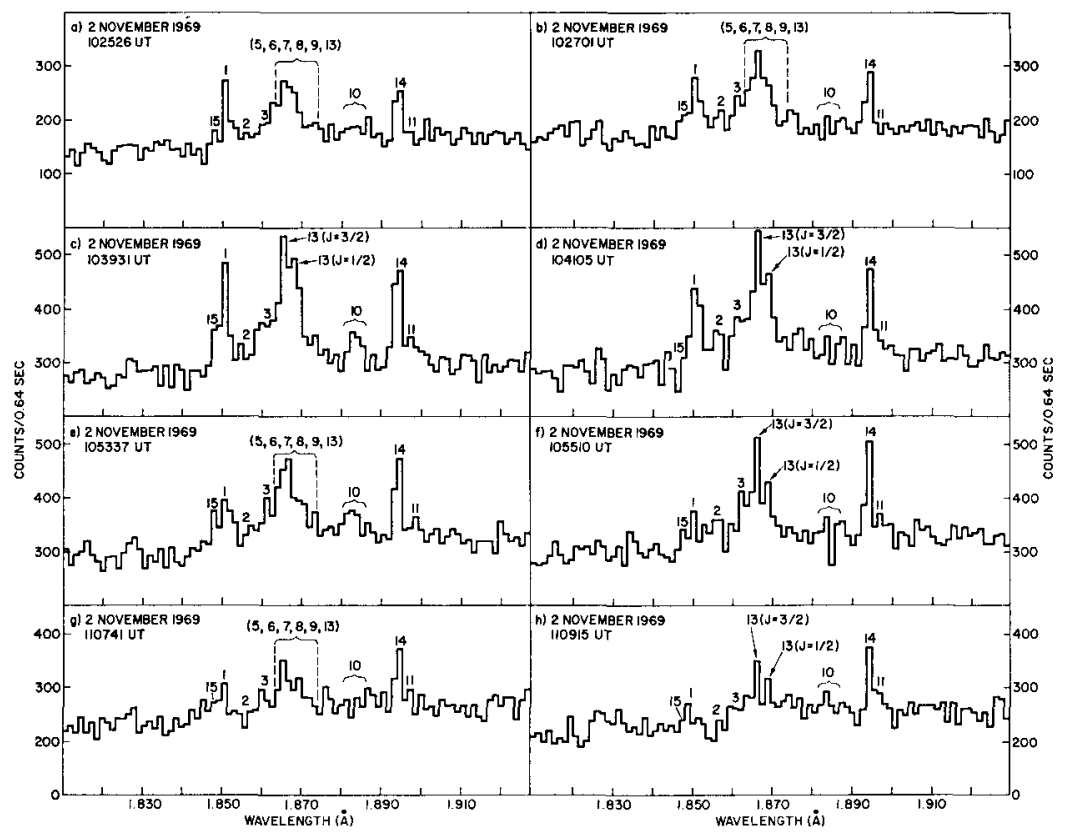

Fig. 29. NRL second order iron spectra from the limb flare on November 2, $1969 \sim 1040$ UT (OSO 6). The numbers above the emission features correspond to the numbered transitions in Table II. 
we are comparing the relative emitting strength of the plasma at $\sim 1.9 \AA$ to emission $\sim 3.7 \AA$, and a decrease in this ratio indicates a decreasing electron temperature.)

The data from the November 2, 1969 event, apart from indicating a general cooling of the flare plasma between $\sim 1030 \mathrm{UT}$ and $1110 \mathrm{UT}$, also show that several temperature regions must exist in the flaring regions. Line ratios of magnesium lines indicate temperatures that are $\sim 15 \times 10^{6} \mathrm{~K}$ less than temperatures found from calcium and sulfur line ratios. Application of instrumental corrections to the raw data will probably not alter the conclusion that the magnesium lines are emitted in a cooler plasma than the silicon and sulfur lines. Therefore, it is possible that the cooler plasma regions actually were heated while the hotter portions cooled. The data presented above do not eliminate this possibility. When the OSO-6 instrumental parameters are determined, it may be possible to intercompare the magnesium, aluminum, silicon, sulfur, and calcium lines, estimate the temperature and emission measure structure of the plasma and investigate changes in this structure during the evolution of the flare.

Finally, we note that the increase in flux of the calcium and iron lines over part of the time interval in which sulfur and silicon line ratios indicate a cooling of the plasma, implies that the emission measure of the hotter plasma regions is increasing during at least part of the cooling phase; a result that is in agreement with similar conclusions based on Horan's analysis of broadband detector data discussed in Section 3A. The NRL data for the November 2 event indicate that the physical structure of the emitting plasma is quite complex, i.e., compare the behavior of the resonance lines of SixIV and Si XIII in Figure 27. Continued study of the soft X-ray spectrum of this flare and others should provide more insight into the dynamical processes governing solar flare plasmas.

\section{HELIUM-LIKE LINES AND RELATED SATELLITE LINES}

Several groups have reported changes in electron density during flares using the forbidden to intercombination intensity ratios (Walker and Rugge, 1970; Doschek and Meekins, 1970; Neupert, 1971a). The Walker and Rugge data refer only to times near flares; the discussion is therefore limited to the NRL and Goddard results.

Neupert (1971a) observed a decrease in the forbidden to intercombination line strengths of SixıI during a large flare on February 27, 1969 compared to the pre-flare value. The ratio varied by $\sim 40 \%$ from a pre-flare value of $\sim 2.6$ to a value of $\sim 1.6$ during the flare. (The actual ratios are subject to error, however, as present instrumentation cannot resolve the lithium-like satellite $\left({ }^{2} D\right)$ that nearly coincides in wavelength with the helium-like forbidden line.) The data used in this analysis are shown in Figure 30. Neupert (1971a) deduces electron densities of $10^{14} \mathrm{~cm}^{-3}$ for the Si XIII lines during this event, and concludes that electron densities generally increase during the intense phases of flares.

This conclusion appears to be inconsistent with previous OSO-4 observations (Doschek and Meekins, 1970) which have been interpreted as showing a decrease in electron density during a large flare on November 16, 1967. However, the NRL observations were made during the cooling phase of the flare, i.e., the flux in the $1 \AA$ to 
PRE-FLARE EMISSION

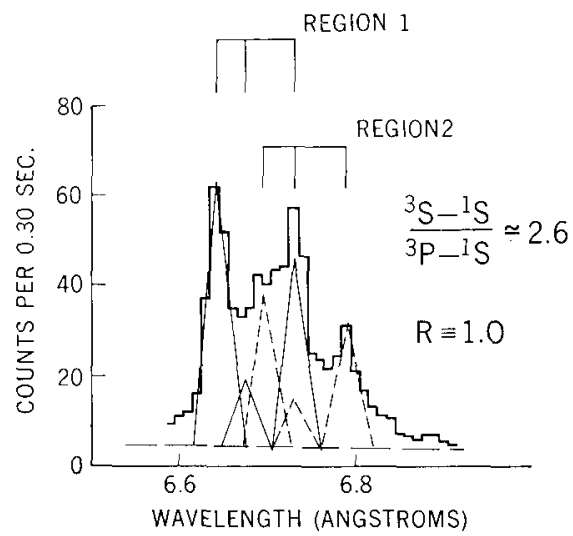

FLARE EMISSION

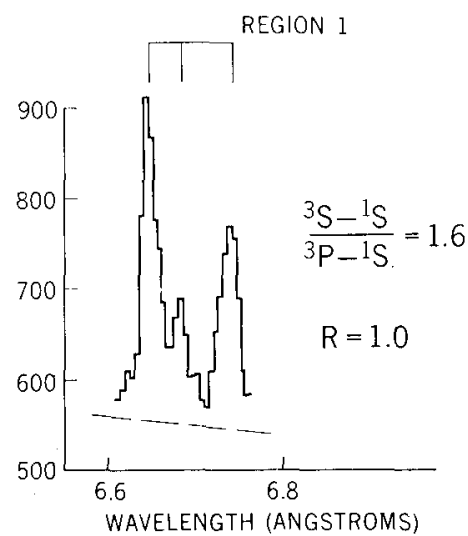

Fig. 30. Pre-flare and flare emission of the resonance, intercombination, and forbidden lines of SixIIr. During the flare, the intensity ratio of the forbidden to intercombination lines has decreased. Note in the preflare spectrum that emission is recorded from two active regions on the Sun. The flare occurs in region 1 and the flux overwhelms contributions from region 2. Spectra courtesy of W. M. Neupert, Goddard Space Flight Center.

$8 \AA$ region was decreasing. Since it is reasonable that flare densities, if indeed higher than active region densities, eventually return to pre-flare values, the NRL observations are not necessarily at variance with the Goddard results. However, we note here that the data presented in Doschek and Meekins (1970) were reduced using only approximate values for the thickness of the mica window on the detector. Therefore, the absolute values of forbidden to intercombination line ratios given in the table in Doschek and Meekins (1970) should be disregarded. Also, the sulfur data presented may suffer from instrumental effects, as the low intensity of the forbidden line in Figure 3 (2146 UT) has not since been observed in other NRL spectra. Nevertheless, the indicated increases in the line ratios of silicon as the flare progresses still appear to be valid observations, but the interpretation of these changes as due to fluctuations of electron density is open to question.

More refined observations are needed to establish accurate forbidden to intercombination line ratios (free from satellite blends); and, indeed, to establish whether or not variations in the forbidden to intercombination line ratios actually occur during flares.

Intensity variations of satellite lines during flares are difficult to detect because of their low intensity and proximity to stronger lines. Doschek et al. (1971b) have studied the helium-like calcium lines and related satellites during the November 2, 1969 flare discussed in Section 5B. Their observations indicated an enhancement of the calcium satellites after the resonance line of Caxix reached peak flux, and a small $(\sim 20 \%)$ increase in the intensity ratio of the combined forbidden and intercombination line to the resonance line of Caxix as the event developed. No statistically significant change in the forbidden to intercombination line was observed. 
Figure 31 shows the time-histories of the resonance, intercombination, and forbidden lines of Caxix, and the time-history of the Caxvil $\left(1 s^{2} 2 s^{2} S-1 s 2 s 2 p{ }^{2} P\right)$ satellite line for the November 2 event. Also shown is the result of subtracting out the intensity of the CaxvIII $\left(1 s^{2} 2 p^{2} p-1 s 2 p^{2}{ }^{2} D\right)$ satellite line from the emission at the wavelength

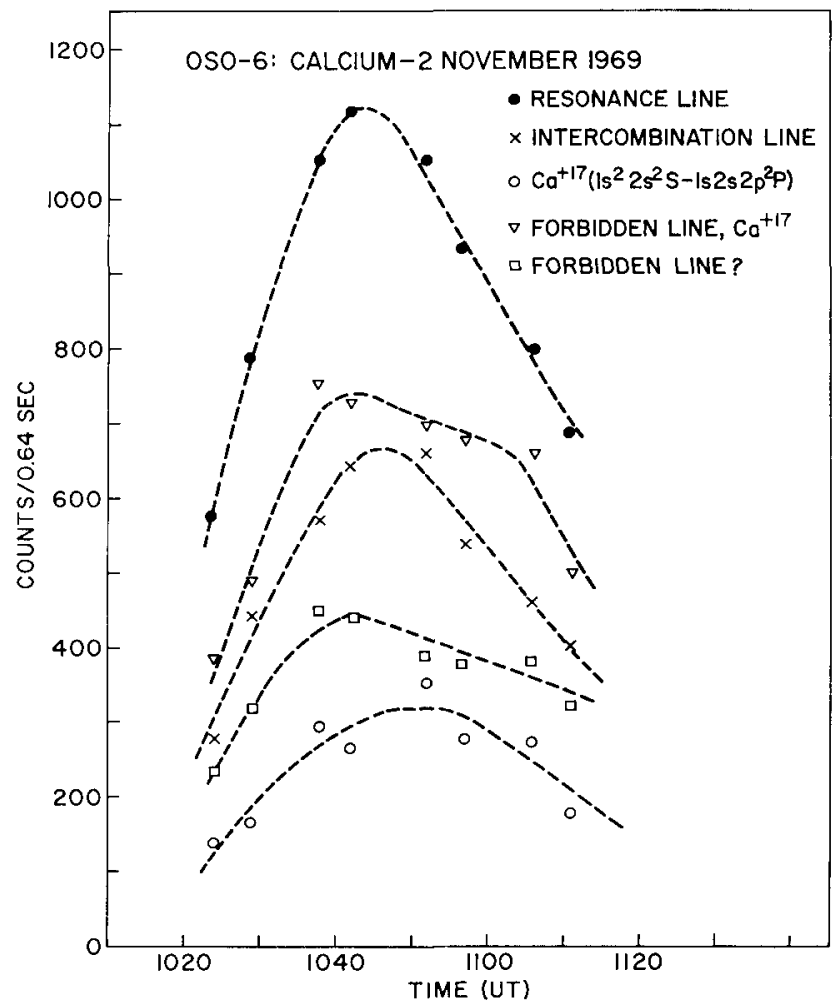

Fig. 31. Time-histories of the resonance, intercombination, and forbidden lines of Caxix from NRL spectra of the November 2, 1969 event (OSO 6). Also shown are the time-histories of the CaxvIII $\left(1 s^{2} 2 s^{2} S-1 s 2 s 2 p^{2} P\right)$ satellite and the forbidden line, modified as described in the text.

of the forbidden line. Since these lines are too well-blended to resolve individual intensities, it was assumed in subtracting out the ${ }^{2} D$ satellite that this line was of the same intensity as the Ca XVIII ${ }^{2} P$ satellite (Gabriel, 1971). Therefore the resultant timeprofile should be regarded with considerable caution.

Figure 31 also shows that the CaxvIII ${ }^{2} P$ satellite peaks later than the helium-like lines, with the exception of the emission at the wavelength of the forbidden line. However, if the ${ }^{2} D$ line is removed as described, then the remaining emission peaks at nearly the same time as the resonance line of CaXIX. A different display of the data is shown in Figure 32. Plotted are the ratios of the forbidden, CaXVIII satellite, and the intercombination line to the resonance line of CaXIX. The data are plotted as a function of scan. The times corresponding to each scan may be found in Figure 31; the 
first scan corresponding to the earliest time. Figure 32 shows more clearly than Figure 31 that the CaxvII satellite was enhanced after the time of peak flux in the Caxix resonance line. However, the error bars are quite large; further observations in other flares are clearly desirable.

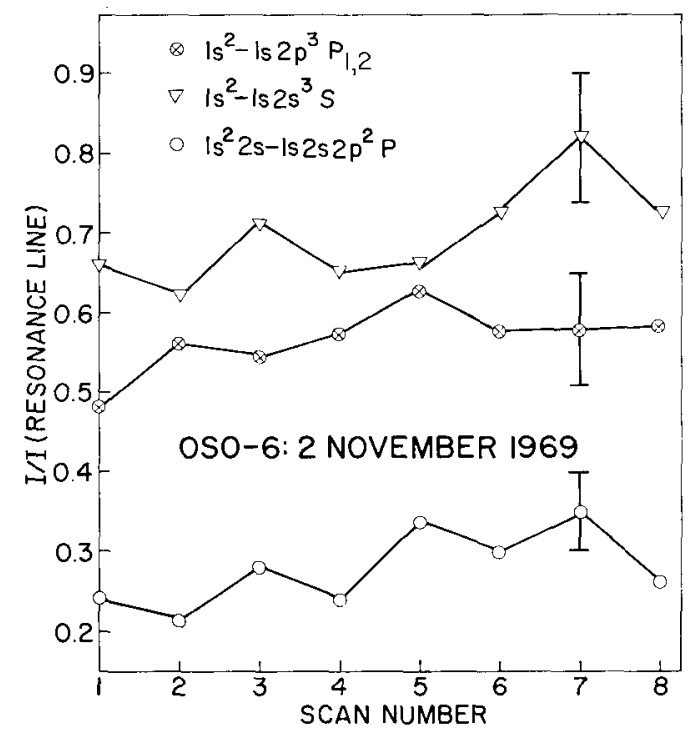

Fig. 32. Ratios of the intensities of the intercombination, and unmodified (see text) forbidden lines of Caxix to the Caxix resonance line from NRL spectra of the November 2, 1969 event (OSO 6).

Data are plotted as a function of scan. The scans correspond to the data points in Figure 31.

Other calcium satellite lines also show an enhancement after the time of peak flux in the CaXIX resonance line for this particular event. Figure 33 shows the lithium-like and beryllium-like satellites on the long-wavelength side of the Caxıx forbidden line. After 1050 UT, a definite enhancement is apparent. However, due to the low intensity of these lines, observations of a number of events will be required to confirm the reality of these variations, and to establish whether or not such variations are present in all flares, or depend on specific plasma conditions. Neupert (1971a) has also observed enhancements of similar lines as in Figure 33 but for magnesium rather than calcium. However, Neupert does not observe an enhancement in intensity after peak flux in the $\mathrm{MgXI}$ lines. Instead, the magnesium satellites are generally enhanced during flare activity and appear to mimic the behavior of the $\mathrm{MgxI}$ lines.

Figure 34 shows the ratio of the sum of the forbidden and intercombination lines to the resonance line of CaxIX. A small increase in this ratio is apparent as the flare progresses. It appears likely that this increase is actually due to the ${ }^{2} D$ satellite discussed above. Doschek et al. (1971b) attributed the enhanced satellite line intensity after peak flux in the Caxix lines as an effect of dielectronic recombination. Further theoretical and observational work is needed, however, before this conclusion can be convincingly established. 


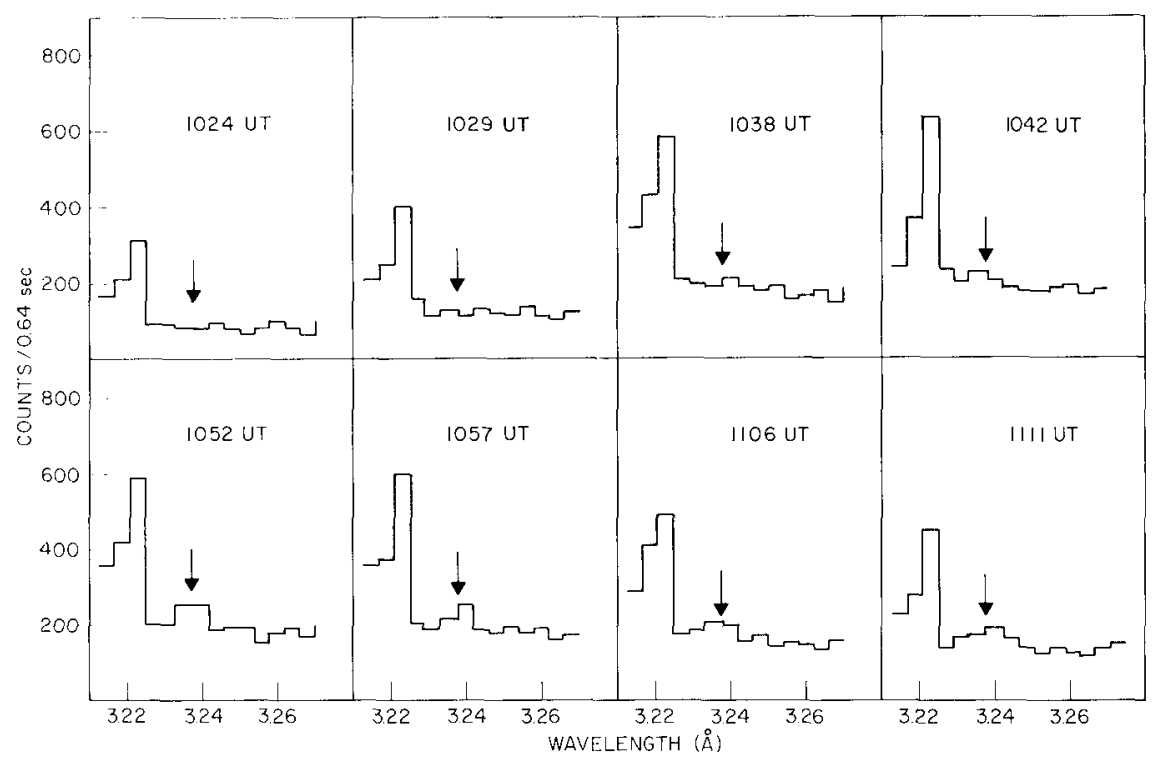

Fig. 33. Inner-shell transitions of calcium seen during the decay phase of the November 2, 1969 event (NRL spectra, OSO 6). The strong lines near $3.22 \AA$ are part of the helium-like line complex (see Table I). The line (or lines) near $3.24 \AA$ are the satellites mentioned. A broadening of this structure by one crystal step toward the forbidden line is especially apparent in the $1111 \mathrm{UT}$ scan, and may be due to Caxvir.

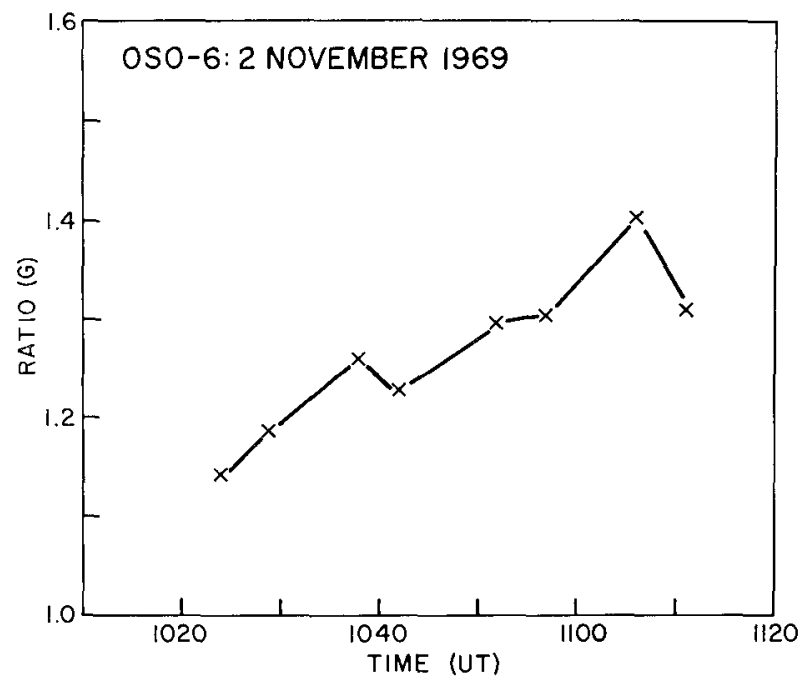

Fig. 34. The intensity ratio of the sum of the forbidden and intercombination lines of Caxix to the resonance line of Caxix from NRL OSO-6 spectra of the November 2, 1969 event. A small increase of the ratio is indicated as the flare progresses. Compare with Figure 31. 
Doschek et al. (1971a) have also observed apparent variations in innershell emission of iron $(\sim 1.9 \AA)$ compared to emission of the Fexxv resonance line. Figure 35 shows second order iron-line data for a number of flares. The numbers above the transitions correspond to the numbered transitions in Table II. A comparison of Figure 35b with Figure $35 f$ (same flare) indicates an increase in Fexxir emission (transition number 10) relative to Fexxv emission (transition number 1) as the flare cools. Similarly, a comparison of Figure $35 \mathrm{e}$ and $35 \mathrm{f}$ (different fiares) indicates a greater amount of FexxII emission relative to Fexxv emission.

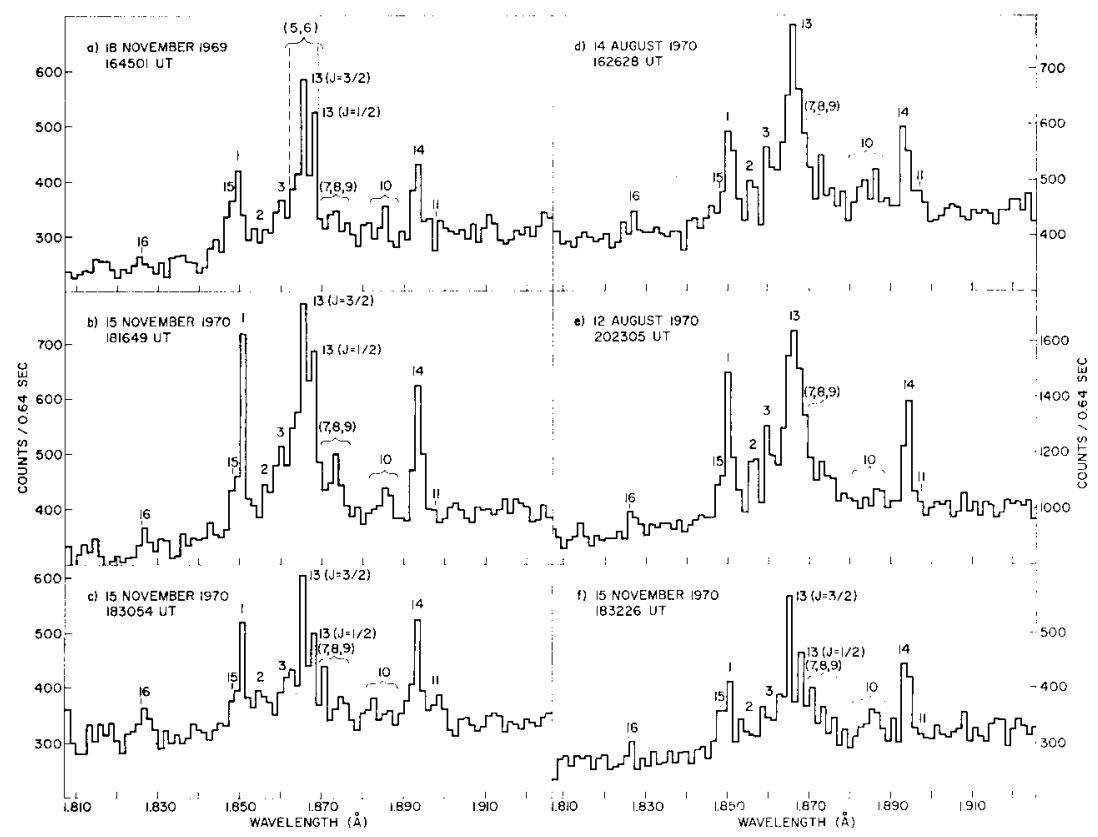

Fig. 35. NRL OSO-6 second order iron spectra of four large flares observed with the LiF spectrometer. The numbers above the emission features correspond to the numbered transitions in Table II. Note the two peaks attributed to the $J=3 / 2, J=1 / 2$ lines of the first excited ${ }^{2} P$ state of Arxvill.

Transitions 5 and 6 are indicated only on Figure 35a, but they also apply to the other spectra.

However, inspection of the spectra of the different flares in Figure 35 between $1.850 \AA$ and $1.874 \AA$ indicates a fundamental similarity in these emission features from flare to flare. For example, we find an average ratio $\sim 0.5$ for the intercombination line to the resonance line of Fexxv. Also, Neupert and Swartz (1970) have not detected changes in the distribution of intensity between FexXV and FexxIV in different flares. It is reasonable to postulate that the basic similarity in the emission features exists because the FexxIv satellite lines are probably primarily produced by dielectronic recombination to Fexxv, and, therefore, if the fractional abundance and flux of Fexxv decreases, the flux in the FexxIv lines must also decrease. However, some of the emission in Fexxiv may be produced by innershell excitation of Fexxiv, and this contribution could vary from flare to flare. 
Qualitative variability of emission among the ionization stages of iron may also be observable in first order during intense flares in which the counting rate in the $1.9 \AA$ feature is large. Figure 36 shows the first order iron emission profiles obtained from the intense flare of August 12, 1970. A second order scan is shown in Figure 35e. Other second order scans did not reveal statistically significant emission features due to the rapid drop in flux of the iron lines during the decay phase of this event. These scans were made $\sim 1037$ UT, four minutes after the scan shown in Figure 36c.

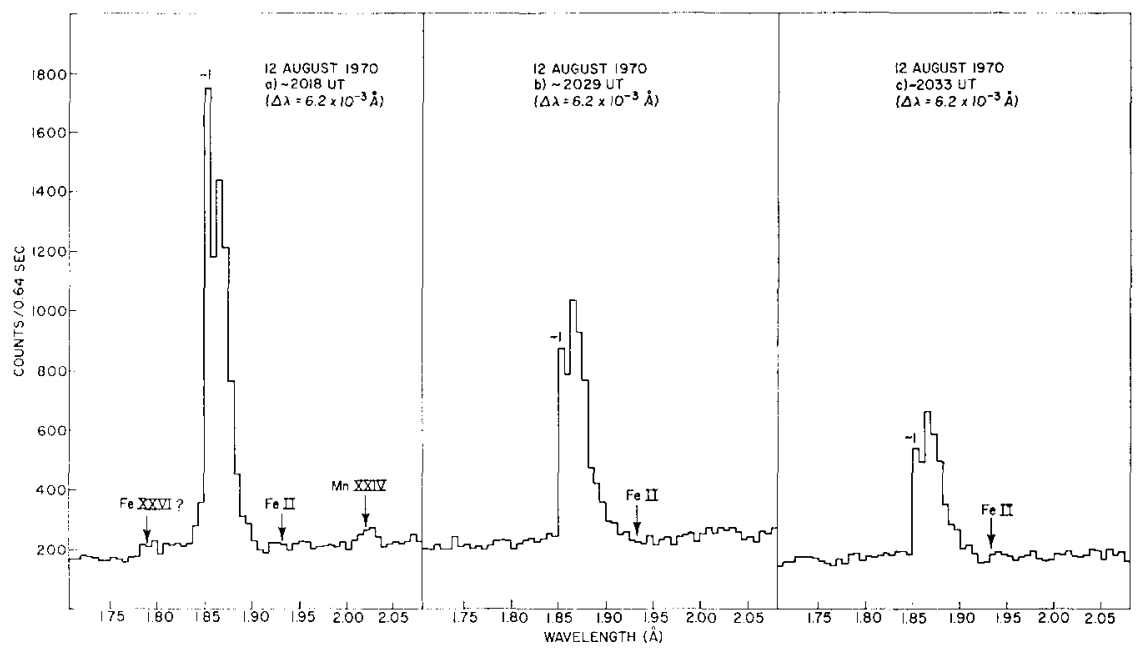

Fig. 36. NRL first order iron spectra (OSO 6) from a large limb flare on August 12, 1970. The feature $\sim 2.02 \AA$ coincides in wavelength with helium-like and $\mathrm{K} x$ inner-shell emission from highly ionized manganese. No significant emission at the wavelength of the FeII K $\alpha$ inner-shell line is apparent.

Two features are noticeable in the scans of Figure 36: (1) the double peak structure is primarily identifiable with the resonance line of Fexxv (short wavelength peak at $1.850 \AA$ ) and the FexxIV satellite features $\sim 1.865 \AA$ (longer wavelength peak); although considerable blending of these features occurs in first order. In Figure 36a, the Fexxv peak at $1.850 \AA$ is greater than the $1.865 \AA$ peak while in Figures $36 \mathrm{~b}$ and $36 \mathrm{c}$ the reverse situation obtains. The shift in maximum intensity of the peaks supports an increase of emission from lower ionization stages of iron relative to Fexxv, and, (2) Figures $36 \mathrm{~b}$ and $36 \mathrm{c}$ exhibit a long wavelength tail to the Fexxv feature which appears more pronounced relative to the $1.850 \AA$ peak than in Figure $36 \mathrm{a}$. However, lines are too numerous and well-blended to attempt a separation in terms of contributions from individual lines. Nevertheless, these data also support a relative increase in emission of lower stages of ionization ( $\gtrsim 15 \%$ of the total emission).

It should be mentioned, however, that recent results presented by Gabriel (1972) at this meeting show that FexxIv satellites are also coincident in wavelength with the FexxIII and FexxII innershell lines. Therefore, the present situation regarding line identifications in this spectral region is unclear. 


\section{Further Comments}

In order to determine the direction of future investigations, it is important to consider the relationship of the soft X-ray flare spectrum to other observations. For this purpose the current picture of the flare phenomenon, resulting from the recent X-ray observations, is briefly summarized below. The part of the discussion concerning hard X-ray $(\lambda \lesssim 1 \AA)$ data is necessarily limited. More detailed discussions can be found in the in the literature cited.

The following sequence of events appears to be characteristic of most intense X-ray flares. A flare usually begins with an enhancement of soft X-ray flux. Shortly thereafter ( $\sim 1 \mathrm{~min})$ a hard X-ray and microwave burst occurs, e.g., De Jager (1968) and the soft X-ray flux begins to increase rapidly. The hard X-rays and microwaves reach maximum flux before the soft X-rays, and begin to decrease in intensity while the soft $\mathrm{X}$-ray flux is still increasing.

The time-histories of the hard X-ray and microwave bursts mimic each other closely in detail, suggesting that these bursts are produced by the same energetic electrons, e.g., Holt and Ramaty (1969). These electrons are generally interpreted as non-thermal in origin (Kahler, 1971; Kane and Donnelly, 1971), and produce hard X-rays by bremsstrahlung and microwaves by the synchrotron mechanism.

From the hard X-ray and microwave data, and assumptions concerning the ambient plasma and magnetic field, it is possible to derive a distribution function for the initial high energy electron population. Present data appear consistent with a power law distribution in energy for the accelerated particles, e.g., Kane and Anderson (1970). However, an arbitrary high energy cut-off is required to explain the observed decrease in flux above $\sim 100 \mathrm{keV}$. The data can also be successfully fitted with Maxwellian distribution functions, although a multithermal source is required (Kane and Anderson, 1970).

Many of the hard X-ray bursts can be subdivided into two components (Frost and Dennis, 1971). The first component, which precedes the second component in time, is impulsive and lasts for $\sim 1 \mathrm{~min}$. The second component is more slowly varying, and its time-profile resembles that of the soft X-ray emission. The total duration of the second component is comparable with the duration of the soft X-ray burst.

The emission spectrum of the second component is fully consistent with a power law distribution in energy for the accelerated particles up to the highest observed energies ( $\sim 250 \mathrm{keV}$; Frost and Dennis, 1971); and is therefore interpreted as non-thermal. Because a non-thermal interpretation of the impulsive first component requires an unexplained high energy cut-off, it has been suggested (Chubb, 1970) that the impulsive burst, or 'spike event' is produced by a multitemperature thermal source at temperatures of $\sim 10^{8} \mathrm{~K}$.

Apparently, some of the energetic particles that produce the impulsive hard X-ray event can precipitate downward heating the chromospheric plasma. During subsequent cooling, extreme ultraviolet emission is produced by radiative recombination and collisional excitation of the chromospheric ions, and this radiation produces an SFD enhancement at the Earth (Kane and Donnelly, 1971). 
Shortly after the impulsive hard X-ray burst reaches peak flux, the soft X-ray event reaches maximum flux and begins to gradually decay. Figures 5 and 6 illustrate the general behavior of the soft X-ray flux, which also resembles the time-profiles of the second component of the hard X-ray burst (Frost and Dennis, 1971). The soft X-ray event is usually accompanied by an $\mathrm{H} \alpha$ flare as described in Section $3 \mathrm{~A}$, which suggests a close connection between these phenomena despite the differences in physical conditions that must exist in the emitting plasmas. It is not presently clear whether cooling by radiation or conduction is more important during the decay phase of the soft X-ray event, because the relative efficiencies of the mechanisms depend heavily on the electron density and geometry of the emitting region (Culhane et al., 1970).

From the broadband observations of hard X-rays, and broadband and crystal spectrometer observations of soft X-rays, the following conclusions appear to be valid. The hard X-ray data indicate that a continuous in situ heating or injection of electrons into the flare region is necessary over the duration of the hard X-ray burst; and a similar continuous injection or heating of electrons is necessary over part of the time of the soft X-ray burst as well (Kahler et al., 1970; Horan, 1971; Lin and Hudson, 1971). (Radiative losses with no heating or injection would produce considerably different X-ray time-profiles than are actually observed.) From the observation that the soft $\mathrm{X}$-ray burst follows the hard X-ray burst closely in time and is much less impulsive in appearance, it has been suggested by many authors, e.g., Neupert et al. (1969) that the energetic electrons that produce the hard X-rays heat the surrounding ambient plasma to the $\approx 30 \times 10^{6} \mathrm{~K}$ temperatures determined by the spectrometer data, thus producing the soft X-ray event, and perhaps indirectly inducing the $\mathrm{H} \alpha$ event as well. In this sense the soft X-ray emission has been regarded as a by-product of the flare mechanism that may give little, if any, information on the actual trigger and acceleration mechanisms of solar flares (Zirin, 1971).

This simplified picture of the flare phenomena, however, is improved when recent photographic and spectroheliographic observations of soft X-ray flares are considered. Figure 37 shows an X-ray photograph of a flare obtained by the AS \& E group (Vaiana et al., 1968). Their analysis of the original negative revealed that the soft X-ray emission is confined to small filaments or threads, which were definitely related to the magnetic neutral line of an $\mathrm{H} \alpha$ filament. Just prior to the onset of the flare, this filament underwent a disparition brusque. Also, the appearance of the X-ray flare strikingly resembles the appearance of the $\mathrm{H} \alpha$ event. The smallest discernable soft $\mathrm{X}$-ray filaments are about $2^{\prime \prime}$ across and separated by $5^{\prime \prime}$. The total volume of the X-ray emitting region is estimated by the AS \& E group as $\gtrsim 10^{28} \mathrm{~cm}^{3}$. From broadband and crystal spectrometer data (Horan, 1971; Meekins et al., 1970; Neupert, 1970), the emission measure of soft X-ray events is on the average $\approx 10^{49} \mathrm{~cm}^{-3}$, implying an electron density for the flare observed by AS \& E of $\approx 3 \times 10^{10} \mathrm{~cm}^{3}$, if the emission is uniformly distributed throughout the emitting plasma. The photograph, however, shows that the most intense part of the emission is confined to ribbons and filaments, indicating that the actual electron density in the filaments may be greater than average values determined from estimated emission measures. 

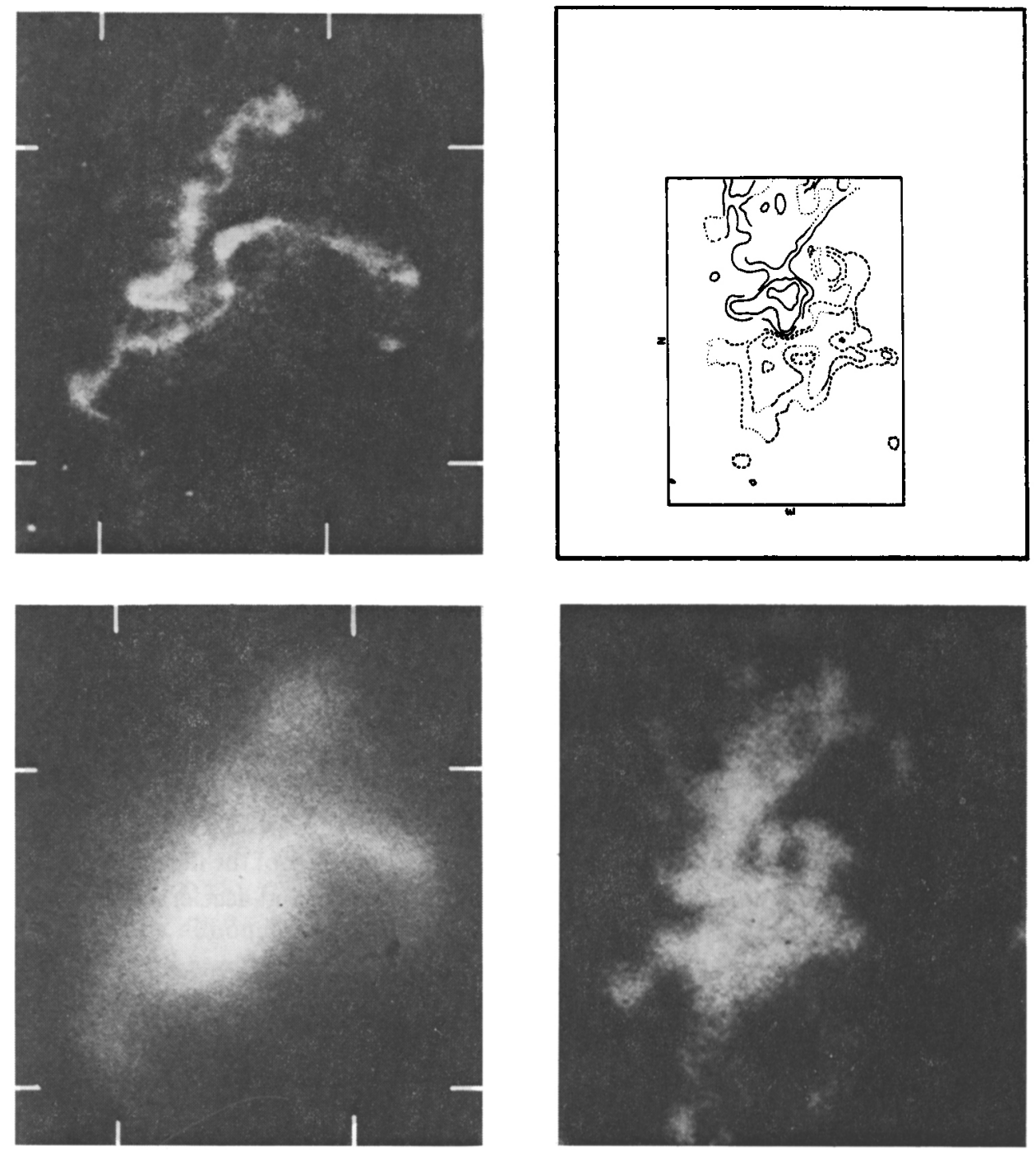

Fig. 37. An X-ray photograph in the $3 \AA$ to $13 \AA$ band of a flare on June 8,1968 . Also shown are photographs in $\mathrm{H} \alpha$ and $\mathrm{CaK}$, and the magnetic field configuration. The X-ray photograph is diagonally across from the magnetic field diagram; the H $\alpha$ photograph is above the X-ray picture and the $\mathrm{CaK}$ photograph is to the right of the X-ray photograph. One inch corresponds to $\sim 1.2$ min. Photograph courtesy of A. S. Krieger, American Science and Engineering.

Recent Russian observations (Beigman et al., 1969; Beigman et al., 1971 b) made from the U.S.S.R. Cosmos-230 spacecraft confirm the existence of filamentary structures in the soft X-ray flare region. The Russian observations reveal that the hotter emitting regions are confined to filament-like structures, and also that these structures exhibit rapid brightness fluctuations in time. The temperatures in the filaments and be- 
tween the filaments vary from $\sim 17 \times 10^{6} \mathrm{~K}$ to $\sim 8 \times 10^{6} \mathrm{~K}$, consistent with crystal spectrometer data.

Other Soviet observations confirm the conclusion that the soft X-ray emitting region has an extremely complicated fine structure with large variations in physical conditions occurring over very short linear distances. Vasiljev et al. (1971) scanned a flare in one dimension with a broadband detector sensitive to X-rays in the $8 \AA$ to $12 \AA$ band, and a quartz crystal spectrometer that monitored emission from the resonance line of FexxV at $1.850 \AA$. The data show that the broadband emission followed the Fexxv emission fairly closely over the flare region, even down to features $\lesssim 20^{\prime \prime}$ in width. Since emission in the $8 \AA$ to $12 \AA$ band must contain considerable contributions from plasma $<15 \times 10^{6} \mathrm{~K}$, and the Fexxv emission is produced by plasma near $25 \times 10^{6} \mathrm{~K}$, these observations appear to be consistent with the Cosmos-230 results indicating that cooler regions are interspersed among the hotter filaments.

Finally, further confirmation of spatial fine structure in X-ray flares was obtained by Thomas and Neupert (1971) from ion chamber ( $2 \AA$ to $8 \AA$, OSO 5) data taken during the total solar eclipse of March 7, 1970. The paths of the Moon during occultation and deoccultation of the flare region were nearly orthogonal, so that a two-dimensional picture of the flare could be constructed. As with the AS \& E photographs and the Russian data, the variations in ion chamber flux could be accounted for by assuming that the $\mathrm{X}$ rays were emitted in a loop-like (filamentary) structure. This structure had a very sharp boundary in one dimension; such that a $50 \%$ change in flux occurred over $\sim 400 \mathrm{~km}$ on the solar disk.

From the above discussion it is clear that a complex magnetic field structure exists in the flare plasma. It also follows that the soft X-ray spectrum is actually a composite spectrum due to emission from all the filaments. In this regard observations such as are illustrated in Figures 23 and 27 are interesting, in that they probably represent changes in large numbers of filaments presumably interconnected by magnetic fields.

Clearly, future spectrometers for the study of solar flares should be designed with high spatial and temporal resolution. Such collimated instruments would be able to observe changes in individual filaments, and from appropriate line ratios, it should be possible to determine the degree of particle entrapment in the filaments, i.e., by determining the relative importance of collisional excitation, dielectronic recombination and radiative recombination. These observations, accompanied by high resolution photographs, should greatly add to our knowledge of the evidently complex interaction between high temperature plasmas and magnetic fields. Since the trigger mechanism that initially induces the flare event must involve an interaction between plasmas and magnetic or induced electric fields, experiments such as these will be of great benefit in shedding light on the acceleration mechanism itself. Furthermore, the variety of plasma conditions found in solar flares provides a fertile field for the investigation of atomic processes in fully ionized plasmas. Hopefully, the observations described in this review and subsequent, future observations will increase our understanding of this important topic.

In summary, current data of soft X-ray flares obtained by crystal spectrometers, 
grazing incidence X-ray telescopes, and spectroheliographs indicate that the soft X-ray flare region is characterized by a complicated set of filamentary structures, apparently interconnected by magnetic fields. The temperatures and electron densities appear to vary strongly in space and time over this network. Temperatures in the filaments range from $\approx 10 \times 10^{6} \mathrm{~K}$ to $\approx 35 \times 10^{6} \mathrm{~K}$, and electron densities may range from $\approx 10^{9} \mathrm{~cm}^{-3}$ to $10^{14} \mathrm{~cm}^{-3}$, although the high values of $\approx 10^{14} \mathrm{~cm}^{-3}$ indicated by the forbidden to intercombination line ratios of heliumlike ions should be regarded with considerable caution. If the flare observed by AS \& E is typical, then total volumes of the flare region are $\approx 10^{28} \mathrm{~cm}^{3}$.

Crystal spectrometer data support laboratory observations (Gabriel and Jordan, 1969a) that dielectronic recombination is an important process in flare plasmas, thus confirming the importance of the process to coronal theory in general. From the observed weakness of innershell transitions in ions with four or more electrons, it appears that non-thermal line excitation of cooler plasma by a hot-electron component is not an efficient mechanism in flare plasmas, and that some degree of thermal equilibrium is achieved in the filaments. This conclusion is supported by the observation of Vasiljev et al. (1971) that the ion temperature of Fexxv in flares is approximately equal to the electron temperature. Future observations of the soft X-ray flare plasma promise to greatly increase our understanding of plasma physics, and therefore, to increase our knowledge of astrophysical phenomena in general.

\section{Acknowledgements}

The author wishes to express his appreciation to A. S. Krieger of American Science and Engineering, W. M. Neupert of Goddard Space Flight Center, and A. B. C. Walker, Jr. of the Aerospace Corporation for kindly sending me examples of their data.

The author owes a special debt of gratitude to John F. Meekins of the Naval Research Laboratory, with whom he has had many stimulating discussions on solar flares and flare spectra.

The author also wishes to thank T. A. Chubb, S. W. Kahler, R. W. Kreplin, J. F. Meekins, and K. G. Widing for critically reading and commenting on the manuscript.

\section{References}

Allen, J. W. and Dupree, A. K.: 1969, Astrophys. J. 155, 27.

Beigman, I. L., Vainshtein, L. A., and Urnov, A. M.: 1971a, preprint.

Beigman, I. L., Vainshtein, L. A., Vacilev, B. N., Zitnik, I. A. Ivanov, V. D., Korneev, V. V., Krutov, V. V., Mandelshtam, S. L., Tindo, I. P., and Shuryghin, A. I.: 1971b, preprint.

Beigman, I. L., Grineva, Yu. I., Mandelshtam, S. L., Vainshtein, L. A., and Zitnik, I. A.; 1969, Solar Phys. 9, 160.

Blake, R. L.: 1968a, private communication.

Blake, R. L.: 1968b, unpublished Ph.D. Thesis, University of Colorado and High Altitude Observatory, NCAR, Boulder, Colorado.

Blake, R. L. and House, L. L.: 1971, Astrophys. J. 166, 423.

Burgess, A.: 1964a, Astrophys. J. Letters 139, 776.

Burgess, A.: 1964b, Mem. Roy. Astron. Soc. 69, Part 1. 
Burgess, A.: 1965, Astrophys. J.Letters 141, 1588.

Burgess, A. and Seaton, M. J.: 1964, Monthly Notices Roy. Astron. Soc. 127, 355.

Burton, W. M. and Wilson, R.: 1961, Proc. Phys. Soc. 78, 1416.

Chubb, T. A.: 1972, in E. R. Dyer (ed.), Solar Terrestrial Physics, 1970, Part I, D. Reidel Publ. Co., Dordrecht, Holland, pp. 99-118.

Cohen, L., Feldman, U., Swartz, M., and Underwood, J. H.: 1968, J. Opt. Soc. Amer. 58, 843. Cowan, R. D.: 1971, private communication.

Culhane, J. L.: 1969, Monthly Notices Roy. Astron. Soc. 144, 375.

Culhane, J. L. and Phillips, K. J. H.: 1970a, Astrophys. J. 160, 309.

Culhane, J. L. and Phillips, K. J. H.: 1970b, Solar Phys. 11, 117.

Culhane, J. L., Sanford, P. W., Shaw, M. L., Phillips, K. J. H., Willmore, A. P., Bowen, P. J., Pounds, K. A., and Smith, D. G.: 1969, Monthly Notices Roy. Astron. Soc. 145, 435.

Culhane, J. L., Vesecky, J. F., and Phillips, K. J. H. : 1970, Solar Phys. 15, 394.

Doschek, G. A. and Meekins, J. F.: 1970, Solar Phys. 13, 220.

Doschek, G. A., Meekins, J. F., Kreplin, R. W., Chubb, T. A., and Friedman, H.: 1971a, Astrophys. J. 170, 573.

Doschek, G. A., Meekins, J. F., Kreplin, R. W., Chubb, T. A., and Friedman, H.: 1971b, Astrophys. J. 164, 165.

Drake, J. F.: 1971, Solar Phys, 16, 152.

Duwez, P.: 1965, Technical Report No. 32 submitted to: U.S. Atm. Energy Commission Contract No. AT(04-3)-221.

Elwert, G. and Haug, E.: 1970, Solar Phys. 15, 234.

Evans, K., Pounds, K. A., and Culhane, J. L.: 1967, Nature 214, 41.

De Feiter, L. D.: 1972, this issue, p. 827.

Flamberg, H.: 1942, Arkiv. Mat. Astron. Fys. 28A, 18.

Freeman, F. F., Gabriel, A. H., Jones, B. B., and Jordan, C.: 1971, Phil. Trans. Roy. Soc. London A270, 127.

Fritz, G., Kreplin, R. W., Meekins, J. F., Unzicker, A. E., and Friedman, H.: 1967, Astrophys. J. 148, L133.

Frost, K. J. and Dennis, B. R.: 1971, Astrophys. J. 165, 655.

Gabriel, A. H.: 1970, private communication.

Gabriel, A. H.: 1971, private communication.

Gabriel, A. H.: 1972, this issue, p. 655.

Gabriel, A. H. and Jordan, C.: 1969a, Nature 221, 947.

Gabriel, A. H. and Jordan, C.: 1969b, Monthly Notices Roy. Astron. Soc. 145, 241.

Garcia, J. D. and Mack, J. E.: 1965, J. Opt. Soc. Amer. 55, 654.

Hauge, O. and Engvold, O.: 1970, preprint, Institute of Theoretical Astrophysics, University of Oslo, Oslo, Norway.

Holt, S. S. and Ramaty, R.: 1969, Solar Phys. 8, 119.

Horan, D. M.: 1971, Solar Phys. 21, 188.

House, L. L.: 1964, Astrophys. J. Suppl. 8, 307.

House, L. L.: 1969, Astrophys. J. Suppl. 18, 21.

Hudson, H. S. and Ohki, K.: 1971, preprint.

De Jager, C. : 1968, Solar Flares and Space Research, Proc. XI COSPAR Symp. Solar Flares, Tokyo, p. 1. Jordan, C.: 1969, Monthly Notices Roy. Astron Soc. 142, 501.

Kahler, S. W.: 1971, Astrophys. J. 164, 365.

Kahler, S. W. and Kreplin, R. W.: 1971, Astrophys. J. 168, 531.

Kahler, S. W., Meekins, J. F., Kreplin, R. W., and Bowyer, C. S.: 1970, Astrophys. J. $162,293$.

Kane, S. R.: 1969, Astrophys. J. Letters 157, L139.

Kane, S. R. and Anderson, K. A.: 1970, Astrophys, J. 162, 1003.

Kane, S. R. and Donnelly, R. F.: 1971, Astrophys. J. 164, 151.

Kreplin, R. W.: 1961, Ann. Geophys. 17, 151.

Kreplin, R. W.: 1970, International Symposium on Solar-Terrestrial Physics, Leningrad, USSR, 1970 May 11-20.

Landini, M. and Monsignori Fossi, B. C.: 1970, Astron. Astrophys. 6, 468.

Lie, T. N. and Elton, R. C.: 1971, Phys. Rev. A3, 865.

Lin, R. P. and Hudson, H. S.: 1971, Solar Phys. 17, 412. 
Meekins, J. F. and Doschek, G. A.: 1970, Solar Phys. 13, 213.

Meekins, J. F., Doschek, G. A., Friedman, H., Chubb, T. A., and Kreplin, R. W.: 1970, Solar Phys. 13, 198.

Neupert, W. M.: 1964, Astrophys. J. 139, 935.

Neupert, W. M.: 1970, in C. J. Macris (ed.), Physics of the Solar Corona, D. Reidel. Publ. Co., Dordrecht, Holland, p. 237.

Neupert, W. M.: 1971a, Solar Phys. 18, 474.

Neupert, W. M.: 1971b, private communication.

Neupert, W. M. and Swartz, M.: 1970, Astrophys. J. Letters 160, L189.

Neupert, W. M., Gates, W., Swartz, M., and Young, R.: 1967, Astrophys, J. Letters 149, L79.

Neupert, W. M., White, W. A., Gates, W. J., Swartz, M., and Young, R. M.: 1969, Solar Phys. 6, 183.

Phillips, K. J. H. and Culhane, J. L.: 1971, Solar Phys. 16, 469.

Pottasch, S. R.: 1964, Space Sci, Rev. 3, 816.

Rugge, H. R. and Walker, A. B. C., Jr.: 1968, Space Res. VIII North-Holland Publ. Co., Amsterdam, p. 439.

Seaton, M. J.: 1959, Monthly Notices Roy. Astron. Soc. 119, 81.

Spitzer, L., Jr.: 1967, Physics of Fully Ionized Gases, Interscience Publishers, New York.

Švestka, Z.: 1970, Solar Phys. 13, 471.

Teske, R. G. and Thomas, R. J.: 1969, Solar Phys. 8, 348.

Thomas, R. J. and Neupert, W. M.: 1971, Bull. $A A S$ 3, No. 1, Part 1, 7.

Thomas, R. J. and Teske, R. G.: 1971, Solar Phys, 16, 431.

Tindo, I. P., Ivanov, V. D., Mandelshtam, S. L., and Shuryghin, A. I.: 1970, Solar Phys. 14, 204.

Tindo, I. P., Ivanov, V. D., Mandelshtam, S. L., and Shuryghin, A. I.: 1971, XIVth Plenary Meeting of COSPAR, Seattle, Washington, U.S.A., 1971.

Tomblin, F. F.: 1971a, private communication.

Tomblin, F. F.: 1971b, Astrophys. J. 171, 377.

Unzicker, A. E.: 1971, private communication.

Vaiana, G. S., Reidy, W. P., Zehnpfennig, T., Van Speybroeck, L., and Giacconi, R.: 1968, Science $161,564$.

Van Regemorter, H.: 1962, Astrophys. 136, 906.

Vasiljev, B. N., Grineva, Yu. I., Zitnik, I. A., Karev, V. I., Korneev, V. V., Krutov, V. V., and Mandelshtam, S. L.: 1971, XIVth Plenary Meeting of COSPAR, Seattle, Washington, U.S.A., 1971.

Walker, A. B. C., Jr. and Rugge, H. R.: 1969, Solar Flares and Space Research, North-Holland

Publ. Co., Amsterdam, p. 102.

Walker, A. B. C., Jr. and Rugge, H. R.: 1970, Astron Astrophys. 5, 4.

Walker, A. B. C., Jr. and Rugge, H. R.: 1971, Astrophys. J. 164, 181.

Wooley, R. v. d. R. and Allen, C. W.: 1948, Monthly Notices Roy. Astron. Soc. 108, 292.

Zirin, H.: 1971, Goddard Space Flight Center Colloquium.

\section{DISCUSSION}

A. H. Gabriel: With regard to your correction of the Ca XIX forbidden line for the ${ }^{2} P-2 D$ Ca XVIII blend, my recent calculations predict an intensity ratio for $\left({ }^{2} P-{ }^{2} D\right) /\left({ }^{2} S-^{2} P\right)$ of 1.6 rather than the 1.0 given by LS-coupling theory. Critical examination of all the solar spectra observed still provide no firm evidence for $\mathbf{K}$ shell emission of ions lower than the lithium-like stage, in contrast to the situation in laboratory spectra. My recent calculations predict for example a weak lithium-like $1 s^{2} 2 p-1 s 2 s^{2}$ satellite doublet in the same region as the expected Fexxir (K) lines. If this suggestion is upheld, then there is a fundamental difference between the iron spectra of laboratory sources and solar flares. High spectral resolution in both would be very valuable.

G. A. Doschek: I agree that higher spectral resolution is necessary. The satellite lines in flare spectra are generally weak, and this makes accurate wavelength measurements difficult. Perhaps the emission observed by NRL at the wavelength of the Fexxil $\mathrm{K}$ line is a blend of Fexxiv and FexxII. The observed emission feature is too broad to be due to a single line.

$Z$. Šestka: Did you compare the X-ray time development with that in $\mathrm{H} \alpha$ ? If these are similar, I don't understand why that in Sixur should deviate from it. 
G. A. Doschek: No. This event was a limb flare and the $\mathrm{H} \alpha$ burst actually occurred behind the limb. However, the X-ray flux in the $2 \AA$ to $8 \AA$ region, which is mostly due to continuum radiation, usually shows a correlation with the development in $\mathrm{H} \alpha$, if a coincident $\mathrm{H} \alpha$ flare is observed. Still, one would not expect all the resonance lines to exhibit a similar time variation. The SixIII line is formed at $\sim 10^{7} \mathrm{~K}$, while the other lines I showed are more indicative of higher temperature plasmas. The $\mathrm{SxvI}$ line has a maximum emission efficiency at $\sim 2 \times 10^{7} \mathrm{~K}$. The continuum emission, however, is due to the contributions from all temperature regions and apparently exhibits an average behaviour that parallels the $\mathrm{H} \alpha$ development.

A. B. C. Walker: One additional remark on the observation of satellite lines from lower stages of ionization than lithium in flares. I have seen a preprint by Dr Neupert which shows spectra from an unusually large flare, and he has identified a number of lines as due to satellites in beryllium-like ions. The evidence he presents seems rather convincing to me.

G. A. Doschek: Yes, I have also seen that preprint. I have incorporated his lines and identifications in the wavelength table included in this review paper. However, I still think higher resolution and more data are required to establish the presence of these ions unequivocally in flare spectra. 